\title{
Abstracts of the 45th Annual Scientific Meeting of the Nutrition Society of Australia ${ }^{+}$
}

\author{
Sandra Iuliano ${ }^{1, *}$, Katherine M. Livingstone ${ }^{2}\left(\mathbb{D}\right.$, Welma Stonehouse ${ }^{3}(\mathbb{D})$, Anthony James ${ }^{4}\left(\mathbb{D}\right.$ and Alison Coates $5{ }^{5}$ \\ 1 Department of Endocrinology, University of Melbourne/Austin Health, West Heidelberg, VIC 3081, Australia \\ 2 Institute for Physical Activity and Nutrition, School of Exercise and Nutrition Sciences, Deakin University, \\ Geelong, VIC 3220, Australia; k.livingstone@deakin.edu.au \\ 3 Health and Biosecurity, Commonwealth Scientific and Industrial Research Organisation (CSIRO), \\ Adelaide, SA 5000, Australia; Welma.Stonehouse@csiro.au \\ 4 Curtin School of Public Health, Curtin University, Bentley, WA 6102, Australia; T.P.James@curtin.edu.au \\ 5 UniSA Allied Health and Human Performance, University of South Australia, Adelaide, SA 5001, Australia; \\ alison.coates@unisa.edu.au \\ * Correspondence: sandraib@unimelb.edu.au; Tel.: +61-438-215-615 \\ + Presented at the 45th Annual Scientific Meeting of the Nutrition Society of Australia, online, \\ 2-3 December 2021.
}

check for updates

Citation: Iuliano, S.; Livingstone, K.M.; Stonehouse, W.; James, A.; Coates, A. Abstracts of the 45th Annual Scientific Meeting of the Nutrition Society of Australia. Proceedings 2022, 80, 2. https://doi.org/10.3390/ proceedings 2022080002

Academic Editor: Maria Emília de Sousa

Published: 1 March 2022

Publisher's Note: MDPI stays neutral with regard to jurisdictional claims in published maps and institutional affiliations.

Copyright: () 2022 by the authors. Licensee MDPI, Basel, Switzerland. This article is an open access article distributed under the terms and conditions of the Creative Commons Attribution (CC BY) license (https:// creativecommons.org/licenses/by/ $4.0 /)$.

\begin{abstract}
The 45th Annual Scientific Meeting of the Nutrition Society of Australia was held virtually from 2-3 December 2021. The theme of the meeting was Nutrition: Opportunities for Nutrition Science in a New Era. Abstracts were submitted from 13 countries. A total of 326 registrants attended the conference, and 134 papers were presented consisting of 6 plenary, 54 oral and 74 poster presentations. This issue presents the proceedings of this meeting in the form of abstracts for papers presented at the conference.
\end{abstract}

Keywords: ageing; agriculture and farming; chronic diseases; communication and education; food security; genomics; gut microbiota; micronutrients; nutrition; public health

\section{Plenary Presentations \\ 1.1. Promoting Health-Enabling Food Retail Environments in Indigenous Communities}

Julie Brimblecombe

Department of Nutrition Dietetics and Food, Monash University, Clayton, Australia

In remote Aboriginal communities of Australia, where food supplies are cut off with the wet season and power outages are frequent, unique and cutting-edge health-enabling initiatives can be observed in community stores. The Amata community council, in the Anangu Pitjantjatjara Yankunytjatjara, took the bold step in 2008 of removing the three top-selling beverages in their store, which reduced sales of sugar by $25 \%$. Store directors of Dungalan community have restricted hours on sales of discretionary products. Many community stores have a policy of not serving school children within school hours, and multi-store organisations, the Arnhem Land Progress Aboriginal Corporation, Mai Wiru, and Outback Stores all have nutrition policies. These health-enabling food retail models deserve recognition and celebration. They provide an example for non-remote Australia of how community, business, and public health goals can be combined in food retail. For millennia, the Indigenous Peoples of Australia had sovereignty of their food systems and pathways, provided for their food and health and wellbeing needs, and passed on food knowledge through the generations. This paper will identify key factors that over the decades have promoted health-enabling food retail in remote communities and show how research in the food retail and health sectors is supporting local-level policy making for strong healthy stores. 


\subsection{The Social Determinants of Diet-Related Health Inequities}

\section{Sharon Friel}

Menzies Centre for Health Governance, School of Regulation and Global Governance, Australian National University, Australia

Redressing the inequities in diet-related health outcomes requires addressing the social and commercial determinants of health. Despite the popular focus on individual risk behaviours as the 'causes' of health outcomes, the social patterning of diet-related health outcomes points toward something about society that is driving these dietary challenges and their unequal social distribution. In this talk, I will describe how inequities in diet-related health are shaped by structural factors including economic, social and health policies and priorities; commercial policies, practices and products; the governance arrangements that develop and implement policy and action; and the social norms and values that pervade society. The evidence presented will reflect on the critical leadership and stewardship roles of nutrition professionals in the pursuit of action on the social and commercial determinants, with many of the necessary actions located outside of nutrition and health.

\subsection{Enhancing Population Nutrition Literacy: First Educate the Educators}

\section{Margaret Miller}

School of Medicine and Health Sciences, Edith Cowan University, Australia

The World Public Health Nutrition Association promotes a public health nutrition workforce pyramid, placing nutrition scientists at the apex and auxiliary workers with direct community contact at the base. Primary school teachers are one such group with enormous potential to influence the nutrition literacy of each generation. Yet little is known of Australian teachers' practices, competence or capacity to deliver. This presentation draws from two studies: the Workforce Practices Questionnaire with 200 primary teachers using structural equation modelling to explore factors that motivate and support teachers to teach nutrition and the 7-year monitoring of the Refresh. ED food and nutrition curriculum support project. Most teachers received no training in nutrition education. For the motivation to teach nutrition and this role, job satisfaction was mainly activated through role legitimacy, pre-empted by feelings of worth and responsibility. Low levels of relevant training, role adequacy and role support were associated with perceived need for support. The use of lesson plans and guidance on curriculum alignment increased Role Legitimacy and Job Satisfaction. Practice-based training increased the Motivation of pre-service teachers. Suitable tertiary pre-service training, curriculum-focused resources and workplace supports could enhance primary teacher engagement in nutrition education. To professionalise this workforce, national guidelines for competency development, support and recognition should be developed and endorsed in consultation with the education sector.

\subsection{Insects: A Sustainable Feed Protein Source for Animal Nutrition}

\section{Kristy Digiacomo}

Faculty of Veterinary and Agricultural Science, The University of Melbourne, Parkville, Australia

Animal products are a valuable source of nutrients and fibre for humans. Modern consumers are increasingly concerned with the environmental impact of animal production. Thus, a key agenda for agricultural production systems is to improve and develop more sustainable practices whereby food and feed can be produced in an environmentally efficient manner. One such process is the bioconversion of organic waste into insects in a no waste system, leading to the development of what is termed a 'circular economy'. Insects are highly efficient and can rapidly convert substrates (such as feed/food waste and manure) into high value products high in protein and fat. Numerous production 
animal species, such as monogastric species and fish, naturally consume insects. Insect production is also estimated to be lower in Green House Gas emissions and have low land and water requirements compared to traditional animal and animal feed production systems. Insect species currently investigated for mass production include black soldier fly larvae (BSFL), mealworms, and crickets. Research into insect production on a mass scale for use as a food or feed source is receiving increased global attention, with multiple companies both in Australia and overseas working to scale up insect production to meet consumer needs. This presentation will introduce the current knowledge of insect rearing for food and feed production.

\subsection{A New Era in Dietary Assessment}

\section{Edith Feskens}

Division of Human Nutrition and Health, Wageningen University, Wageningen, The Netherlands

The assessment of individual dietary intake is essential for nutrition science as well as dietary practice. The most common methods in research are $24 \mathrm{HR}$ recall and FFQ, but they are all based on self-report and have their limitations: for example, underreporting of intake due to forgotten foods and portion underestimation or reliance on a food list tailored for specific populations in case of an FFQ. These limitations seriously hamper the field, for example, in the interpretation of epidemiologic studies and the inclusion criteria. However, new methods are under development using new technologies such as the use of photographs to document portion size or smart devices to measure swallowing or arm movements. Although many issues still need to be solved, improvements have already been made based by using smartphones, such as the new 2-h recall, as developed by our group, Traqq. In addition, biomarkers are useful adjunct measures to validate self-reporting and/or to combine with self-reporting to better estimate exposure or can be used as such instead of self-reports. Based on advances in metabolomics, the current set of biomarkers (originally classified as recovery or concentration biomarkers) are now being expanded to include food intake biomarkers. Examples from food metabolomics, including markers of fermented foods, will be discussed.

\subsection{Precision and Personalised Nutrition: Navigating Challenges and Opportunities for Nutrition Science in a Big Data Era}

\section{Katherine M. Livingstone}

Institute for Physical Activity and Nutrition, School of Exercise and Nutrition Sciences, Deakin University, Geelong, Australia

The Australian Academy of Science's decadal plan for nutrition identifies the field of precision and personalised nutrition as an opportunity to advance nutrition science. In this field, the complex and varied individual characteristics that influence dietary behaviours are used to design tailored, or personalised, dietary interventions to improve health. A recent systematic review suggests that personalising dietary advice helps individuals to improve their dietary intake more than generalised "one-size-fits-all" dietary recommendations. However, there remain many unanswered questions for how best to design and implement personalised nutrition approaches at scale and in a manner that does not increase health inequities. This talk will explore the challenges and opportunities for precision and personalised nutrition science in a big data era. I will provide an overview of recent research, focusing on my findings from the Food4Me Study, the largest pan-European randomized controlled trial of personalised nutrition to date. This talk will also spotlight emerging science on the interactions between individual characteristics, such as diet, genetics, microbiome and environment, highlight the use of self-monitoring technologies, such as wearable devices, and discuss the ethics of using big data and machine learning to inform the design of dietary interventions and public health policy. 


\section{Oral Presentations}

\subsection{Quality and Accuracy of Online Nutrition-Related Information: A Systematic Review}

Emily C. Denniss, Rebecca Lindberg and Sarah A. McNaughton

School of Exercise and Nutrition Sciences, Deakin University, Geelong, Australia

Consumers are increasingly using online environments (e.g., websites, social media) to obtain nutrition-related information. The aim of this systematic review was to synthesise evidence on the quality and accuracy of nutrition-related information in online environments. Five databases were searched in January 2020. Content analysis studies focused on the quality and/or accuracy of nutrition-related information in online environments published in English between 1989 and 2020 were eligible for inclusion. Data about information quality and/or accuracy were extracted. A total of 65 studies were included. In total, 53 studies examined websites (18 evaluated quality, 18 accuracy and 17 both quality and accuracy), 11 studies examined social media ( 1 evaluated information quality, 4 accuracy and 6 both quality and accuracy) and 1 study examined both websites and social media (quality and accuracy). Preliminary findings show that information quality and accuracy was highly variable. Most websites or social media posts included a mixture of high- and low-quality and/or accurate and inaccurate information. Variation in the quality and accuracy of nutrition-related information in online environments raises concerns about its potential to cause harm, as consumers may find it difficult to discern which information they should utilise to inform health actions.

\subsection{The Knowledge, Messaging and Selection of Whole-Grain Foods: An Australian Consumer Perspective}

\section{Katrina R. Kissock ${ }^{1,2}$, Elizabeth P. Neale ${ }^{1,2}$ and Eleanor J. Beck 1,2 \\ 1 University of Wollongong, Wollongong, Australia \\ 2 Illawarra Health and Medical Research Institute, Wollongong, Australia}

Whole-grain consumption is encouraged within dietary guidelines, although global intake remains low. Improving whole-grain intake requires consideration of the factors affecting consumer grain food choices. The current study aimed to investigate consumer understanding and identification of whole-grain foods and labelling and to further explore the factors influencing consumer grain food choices. Data were collected through online semistructured focus groups, each consisting of 3-5 participants. Focus group questions were open-ended and explored consumer grain food choices, identification of whole-grain foods, perceptions on current front-of-pack labelling and opinions on hypothetical front-of-pack labelling. The data were analysed using inductive thematic analysis. Across participants ( $n=43 ; 10$ focus groups), there was a lack of understanding and uncertainty around defining and identifying a whole-grain food. Scepticism around labelling was a prominent factor affecting consumer understanding. This influenced the preferences consumers had towards whole-grain labelling, with most preferring 'whole grain' within the product name or a statement on the whole-grain percentage within a product. Statements such as 'contains whole grain' promoted scepticism. Familiarity, price and taste also influenced consumer grain choice. Consistent and simple labelling and further education is needed to increase consumer understanding around whole-grain foods. This may assist and guide consumers towards greater whole-grain intake.

\subsection{Aspiring Nutritionists' Study: Exploring Student's Pre-Professional Identity, Motivations and Expectations Relating to Careers in Nutrition}

Libby Swanepoel, Angela Cleary and Anthony Villani

University of the Sunshine Coast, Maroochydore DC, Australia

Universities play a crucial role in the formation of pre-professional identity and shaping students for the future workforce. To adequately prepare nutrition students for 
increasingly diverse workforce environments, we must first understand the attitudes, expectations, and career plans for aspiring nutritionists. This qualitative study employed a general inductive inquiry approach utilising focus group discussions to explore the perspectives of first year nutrition students. Conventional content analysis was undertaken using NVivo, whereby discussion transcripts were coded and categorised following an inductive approach, allowing key themes to emerge. Participants $(n=44)$ were mostly female $(87.5 \%)$ with a mean age of 27.5 years. Benevolence and a passion for food were key drivers for undertaking a career in nutrition. Professionalism, communication, willingness to learn and a non-judgemental attitude were identified as core skills, whilst positivity, critical thinking, compassion and creativity emerged as key personal attributes. Students saw themselves working in a limited variety of areas, including the food industry, educational roles, sustainable food systems and public health. There is a need to align core competencies with factors that students identify as priorities for practice. Further marketing the diversity of nutrition practice options for people considering the profession is also warranted.

\title{
2.4. The Relationship between Diet, the Gut Microbiome and Metabolic Health: Consumer Understandings and Perceptions
}

\section{Georgina Williams and Eleanor Beck}

University of Wollongong, North Wollongong, Australia

The influence of diet on the gut microbiome and subsequent health effects is increasingly of interest across popular media and scientific literature. Understanding consumers' perceptions on this topic aimed to inform research and tailor health recommendations to improve health outcomes. We conducted online focus groups (adults; no formal medical or nutrition training) questioning consumers' understanding of 'gut health' and associated health outcomes and their perceptions of evidence-based health recommendations. Discussions were transcribed verbatim, and inductive thematic analysis was completed in duplicate. Fourteen focus groups were conducted ( $n=38,15$ males, 23 females). Individuals considered 'gut health' important for 'overall wellbeing' despite the role of the gut microbiome being poorly understood. Participants named fermented foods or probiotic supplements in preference to core food groups as beneficial to 'gut health'. A barrier to dietary changes to promote gut health was the perception that changes were required for curative, rather than preventative, health measures. While current research does not infer that dietary choices for 'gut health' differ to general dietary guidelines, further research is required to enable practical and evidence-based recommendations to improve consumer understanding of the potential role of the gut microbiome in preventative healthcare and promote dietary choices consistent with dietary guidelines.

\subsection{Chrononutrition as a Potential Target for Young Women (18-25 Years Old) with Overweight and Obesity}

\author{
Isabel Young ${ }^{1}$, Helen Parker ${ }^{2}$, Kate Steinbeck ${ }^{3}$, Natalie Crino ${ }^{2}$ and \\ Helen $\mathrm{O}^{\prime}$ Connor ${ }^{2}$ \\ 1 Faculty of Medicine and Health, The University of Sydney, Sydney, Australia \\ 2 Faculty of Health Sciences, University of Sydney, Sydney, Australia \\ 3 Faculty of Medicine and Health, The Clinical School at The University of Sydney \\ Children's Hospital Westmead, Sydney, Australia
}

The timing of energy intake as a target for weight loss interventions has increased in popularity in recent years, with earlier weighting of daily energy intake being associated with improved health and weight. This cross-sectional study aimed to explore the eating patterns of young women (18-25 y) with overweight/obesity to determine if meal timing may be an appropriate target for assisting weight loss. Young women $(n=28$; mean age: $22.6 \mathrm{y}$; BMI: $32.2 \mathrm{~kg} / \mathrm{m}^{2}$ ) completed 3-day weighed food records. The average time of the first meal was 9:12 a.m. (range: 5:15 a.m.-12:40 p.m.) with the last meal at 10:43 p.m. (range: 5:43 p.m.-2 a.m.) and an average eating window of $11.5 \mathrm{~h}$, with $21 \%$ of participants 
eating at >12 a.m. Mean total daily energy intake was $9174 \mathrm{~kJ}$, with intakes of total energy, discretionary food, fat, saturated fat, protein and carbohydrates being significantly greater $>2$ p.m. $(p<0.05)$. Diet quality was poor, with participants not meeting requirements for vegetable, fruit and dairy, consuming mostly refined grains, and averaging 6 servings of discretionary foods/day, including at least one takeaway meal. The study shows that young women with overweight/obesity have poor quality diets characterised by overall energy intake significantly weighted towards the afternoon/evening. Therefore, weight loss interventions aiming to more evenly distribute energy intake may be successful in this group.

2.6. What Diet, Physical Activity and Behavioural Strategies Are Used by Women with Polycystic Ovary Syndrome and Where Are They Sourced From?

Stephanie Cowan ${ }^{1}$, Angela Grassi ${ }^{2}$, Lynn Monahan-Couch ${ }^{2}$, Yvonne Jeanes ${ }^{3}$, Siew Lim ${ }^{1}$, Stephanie Pirotta ${ }^{1}$, Jeffrey Harris ${ }^{2}$, Caroline McGirr ${ }^{1}$ and Lisa Moran ${ }^{1}$

1 Monash University, Clayton, Australia

2 West Chester University of Pennsylvania, West Chester, USA

3 University of Roehampton, Roehampton, London, UK

Polycystic Ovary Syndrome (PCOS) is a complex endocrine disorder, affecting 13\% of reproductive-aged women. While lifestyle management is the first-line treatment, women experience challenges with implementation. The primary aims of this cross-sectional study were to identify the types and sources of dietary and physical activity (PA) interventions implemented by women with PCOS ( $n=1167,18-45$ years). A secondary aim was to understand how self-management strategies are used to support behaviour change. An online questionnaire was disseminated via a PCOS consumer-based website (May 2015 to May 2016). A quarter or less of women (diet 25\%; PA 14\%) source lifestyle advice from healthcare professionals (medical clinicians or dietitians) compared to $>50 \%$ (diet 59\%; PA $67 \%$ ) using alternative sources, namely, from online platforms. While only $33 \%$ of women followed formal dietary guidelines, 57\% implemented a 'special diet' to manage their condition, many of which were inconsistent with evidence-based practice. Participants also displayed a low level of engagement with self-management behaviours, including goal setting and positive self-talk. Findings emphasize the need to increase women's engagement with health professionals when seeking lifestyle advice for PCOS management. Future research should consider innovative education strategies that are better able to satisfy the unique learning needs of this demographic.

2.7. Anthocyanins Attenuate Vascular and Inflammatory Responses to a High Fat High Energy Meal Challenge in Overweight Older Adults: A Cross-Over, Randomised, Double-Blind Clinical Trial

Vinicius do Rosario ${ }^{1}$, Courtney Chang ${ }^{1,2}$, Jaclyn Spencer ${ }^{1}$, Thilani Alahakone ${ }^{1}$, Steven Steven Roodenrys ${ }^{1}$, Monique Francois ${ }^{1,2}$, Katrina Weston-Green 1,2,3, Nadine Macha née Hölzel ${ }^{4}$, David Nichols ${ }^{5}$, Katherine Kent ${ }^{5}$, David Williams ${ }^{6}$, Ian M.R. Wright ${ }^{7}$ and Karen Charlton 1,2

1 School of Medicine, Faculty of Science, Medicine and Health, University of Wollongong, Australia

2 Illawarra Health and Medical Research Institute (IHMRI), Wollongong, Australia

3 Molecular Horizons, University of Wollongong, Wollongong, Australia

Central Sciences Laboratory, University of Tasmania, Hobart, Australia

Centre for Rural Health, University of Tasmania, Launceston, Australia

Department of Agriculture and Fisheries, Queensland Government, Brisbane, Australia

James Cook University, College of Medicine and Dentistry, Cairns, Australia

Postprandial metabolic imbalances are indicators of developing cardiovascular disease (CVD). This crossover, randomised, controlled, double-blind clinical trial investigated the 
effects of food-anthocyanins on vascular function and CVD-associated biomarkers following a high-fat, high-energy (HFHE) meal challenge in overweight older adults. Participants $\left(n=16\right.$, mean age 65.9 SD 6.0 and BMI $30.6 \mathrm{~kg} / \mathrm{m}^{2}$ SD 3.9) consumed a HFHE meal with a $250 \mathrm{~mL}$ dose of anthocyanins-rich plum or control juice. Blood samples and blood pressure measures were collected at baseline, $2 \mathrm{~h}$ and $4 \mathrm{~h}$ following the HFHE meal. Vascular and microvascular function were evaluated at the baseline and $2 \mathrm{~h}$ timepoints. Participants had a higher $2 \mathrm{~h}$ flow-mediated dilatation $(+1.14 \%)$ and microvascular post-occlusive reactive hyperaemia $(+0.10$ perfusion units per $\mathrm{mmHg})$ when allocated to the anthocyanin compared to the control arm ( $p=0.019$ and $p=0.049$, respectively). C-reactive protein was lower $4 \mathrm{~h}$ postprandially in the anthocyanins $(1.80 \mathrm{mg} / \mathrm{L}$, IQR 0.90$)$ vs. control arm (2.30 mg/L, IQR 1.95) ( $p=0.026)$. No significant postprandial differences were observed for blood pressure and other CVD-associated biomarkers. Fruit-based anthocyanins attenuated the postprandial detrimental effects of an HFHE challenge on vascular and microvascular function and inflammatory biomarkers in overweight older adults.

2.8. Whole-Fat Dairy Products Do Not Adversely Affect Adiposity or Cardiometabolic Risk Factors in Milky Way Study Kids: A Double Blind Pilot RCT
Analise Nicholl ${ }^{1}$, Kane E. Deerin ${ }^{1,2}$, Katherine Evelegh ${ }^{1}$, Philippa Lyons-Wall ${ }^{1}$, David Lawrence ${ }^{3}$, Trevor A. Mori ${ }^{4}$, Mario Kratz ${ }^{5,6}$ and Therese O'Sullivan ${ }^{1}$
1 Institute for Nutrition Research, Edith Cowan University, Joondalup, Australia
2 Digital Solutions Division Information and Data Manager Information Management,
ACT Govt Dept of Health COVID-19 Response, Canberra, Australia
3 Faculty of Arts, Business, Law and Education, Graduate School of Education,
University of Western Australia, Perth, Australia
4 Medical School, University of Western Australia, Perth, Australia
5 Division of Public Health Sciences, Fred Hutchinson Cancer Research Center,
Seattle, USA
6 Department of Epidemiology, University of Washington, Seattle, USA

Limited evidence supports public health guidelines that children over two years consume mostly reduced-fat dairy. We aimed to investigate effects of whole-fat vs. reducedfat dairy intake on markers of cardiometabolic risk in healthy 4-6-year-olds. Children ( $n=49 ; 5.2 \pm 0.9$ years; $47 \%$ female) who habitually consumed whole-fat dairy were randomised in a double-blind fashion to remain on whole-fat dairy or change to reducedfat products for three months, while maintaining their habitual diet. Study endpoints included body fat percentage and serum markers of cardiometabolic health risk; diet was assessed via 3-day weighed food records. Pre- and post-intervention results were compared using linear mixed models, adjusted for growth, age and sex. Dairy fat intake reduced by an adjusted $12.9 \pm 4.1 \mathrm{~g} /$ day in the reduced-fat vs. the whole-fat dairy group (95\% CI: $-21.2,-4.6 ; p=0.003)$, but dietary energy intakes remained similar $(p=0.936)$. We found no significant differential changes in any measure of cardiometabolic health risk. Those consuming reduced-fat dairy increased their dietary sodium by an adjusted $241 \mathrm{mg} /$ day $(15.2 \%)$ compared to the controls. Hence, while changing to reduced-fat dairy does lower dairy fat intake, it does not affect markers of adiposity or cardiometabolic disease risk in healthy children.

\title{
2.9. EatSmart: A Pilot Trial of a Digitally-Delivered Program Promoting Healthy Eating Behaviours among Disadvantaged People with Type 2 Diabetes
}

\author{
Nazgol Karimi ${ }^{1}$, David Crawford ${ }^{1}$, Rachelle Opie ${ }^{1,2}$, Stella O'Connell ${ }^{1}$, \\ Shane P Hamblin ${ }^{3,4}$, Cheryl Steele ${ }^{5}$, Ralph Maddison ${ }^{1}$, Bodil Rasmussen ${ }^{6,7,8}$, \\ Ashley $\mathrm{H} \mathrm{Ng}^{9}$ and Kylie Ball ${ }^{1}$ \\ 1 Institute for Physical Activity and Nutrition, Deakin University, Geelong, Australia \\ 2 IMPACT, Food \& Mood Centre, Deakin University, Geelong, Australia \\ 3 Diabetes and Endocrinology Centre, Sunshine Hospital, Melbourne, Australia \\ 4 Department of Medicine-Western Precinct, University of Melbourne, Melbourne, Australia
}


5 Diabetes Education Services, Sunshine Hospital, Melbourne, Australia

School of Nursing and Midwifery, Deakin University, Geelong, Australia

Centre for Quality and Patient Safety Research, Western Health Partnership, Sunshine Hospital, Melbourne, Australia

8 Faculty of Health and Medical Sciences, University of Copenhagen, Copenhagen, Denmark

9 Department of Dietetics, Human Nutrition and Sport, La Trobe University, Melbourne, Australia

Digitally delivered interventions show promise for promoting healthy eating behaviours. However, little is known about their effectiveness among socioeconomically disadvantaged populations. This study aimed to explore the potential effectiveness of EatSmart, a 12-week, web- and mobile-delivered healthy eating behaviour change support program for disadvantaged people with T2D. EatSmart was a pre-post design pilot study. Sixty adults with T2D who had a healthcare card/pension were recruited. The program included a website with 6 progressive skill-based modules and 36 text messages. Outcome data were self-reported in surveys completed at three time-points: baseline (T1); immediately post-intervention (T2); and 6-months post-intervention (T3). Participants' mean age $( \pm S D)$ was $53.83 \pm 12.1$ years, and $60 \%$ were non-Australian-born. The results of the linear mixed models showed that, compared with T1, mean daily vegetables consumption increased by 0.94 servings/day $(p<0.001)$ at T2, with this effect being sustained, although smaller, at T3 (an additional 0.85 servings $/ d ; p<0.001$ ). Fruit consumption also increased by 0.28 and 0.49 servings / day at T2 and T3, respectively, although this increase was only significant at T3 $(p<0.05)$. This study provides evidence of the general effectiveness of the EatSmart program for participants. A full-scale randomised controlled trial is needed to confirm the findings from this pilot study.

\subsection{Lifestyle Interventions for Diabetes Prevention by Ethnicity: A Systematic Review of Intervention Characteristics Using the TIDieR Framework}

Mingling Chen ${ }^{1}$, Gebresilasea Gendisha Ukke ${ }^{1}$, Lisa J. Moran ${ }^{1}$, Surbhi Sood ${ }^{1}$, Christie J. Bennett ${ }^{2}$, Mahnaz Bahri Khomami ${ }^{1}$, Pilvikki Absetz ${ }^{3}$, Helena Teede ${ }^{1}$, Cheryce L. Harrison ${ }^{1}$ and Siew Lim $^{1}$

1 Monash Centre for Health Research and Implementation, Monash University, Clayton, Australia

2 Department of Nutrition, Dietetics and Food, School of Clinical Sciences, Monash University, Notting Hill, Australia

3 Faculty of Social Sciences, Tampere University, Tampere, Finland

Lifestyle intervention is effective in preventing type 2 diabetes mellitus (T2DM), but the efficacy of intervention components across different ethnic groups is less clear. This systematic review examined the effects of intervention characteristics of lifestyle interventions on diabetes incidence and weight loss by ethnicity using the Template for Intervention Description and Replication (TIDieR) framework. MEDLINE, EMBASE and other databases were searched (to June 2020) for randomised and non-randomised controlled trials on lifestyle interventions (diet and/or physical activity) in adults at risk of T2DM. Ethnicity was categorized into European, South Asian, East and Southeast Asian, Middle Eastern, Latin American and African groups. In total, 45 studies $(n=18,789)$ were included in the systematic review and 41 studies in the meta-analysis. The meta-analysis showed that a high number of intervention sessions was significantly associated with a greater reduction in diabetes incidence $(p=0.043)$ and weight $(p=0.015)$, while other intervention characteristics including intervention provider and delivery format did not alter the outcomes (all $p>0.05)$. When stratified by ethnicity, long-term interventions $(\geq 12$ months) were associated with significant diabetes risk reduction for all ethnic groups, while short-term interventions ( $<12$ months) were more effective in weight loss in most ethnic groups. There may be ethnic preferences for the optimal number of intervention sessions. 


\subsection{Liking of Salt Is Associated with Anxiety, Stress and Depression}

Celeste Ferraris, Tamara Bucher, Chris Scarlett and Emma Beckett

School of Environmental \& Life Sciences, University of Newcastle, Ourimbah, Australia

Early research has shown relationships between sodium intake, serum depletion, and depression and variations in salt tasting in anxiety and stress. Variations in liking and the perceived intensity of salty foods in stress, anxiety, and depression have not been studied. Therefore, an Australian population survey ( $n=432$, after exclusion for abnormal taste) was conducted. Participants rated perceived intensity and liking of salt index foods on a General Labelled Magnitude Scale (1-100) and completed the Depression, Anxiety, and Stress Scale (DASS-21) to measure these states. Standard least squares regression (post hoc Tukey's HSD) was used to compare means between groups. Adjustments were made for age, sex, education, income, and smoking. Those with moderate stress level scores had higher salt liking than those with normal stress scores (67.2 vs. 60.1, $p=0.01)$. Salt liking was also higher in participants with severe anxiety scores (67.8 vs. 60.0, $p=0.003$ ) or severe depression scores (67.8 vs. 60.1, $p=0.009$ ) than in those with normal scores, with results maintained after adjusting for all confounders. No relationships between perceived salt intensity and mood states were found. Liking of salty foods increases in stress, anxiety, and depression.

\subsection{Food Addiction and Dietary Intake in Major Depressive Disorder}

Margaret McGowan ${ }^{1}$, Karen Walton 1,2, Theresa Larkin 1,2, Karen Charlton 1,2, Asmahan Elgellaie ${ }^{1,2}$ and Susan Thomas ${ }^{1,2}$

1 School of Medicine, Faculty of Science, Medicine and Health, University of

Wollongong, Wollongong, Australia

2 Illawarra Health and Medical Research Institute, Wollongong, Australia

Depression has been associated with excess body weight and poorer diet quality, suggesting a possible role of food addiction (FA). The aim of this study was to determine the correlation between major depressive disorder (MDD) and FA, and the differences in dietary intakes between MDD participants with and without FA. A total of 59 adults meeting the DSM-5 criteria for MDD, 25 with FA, and 57 healthy controls, 2 with FA, completed the DASS-21, the Yale Food Addiction Scale 2.0, and a diet history. A strong positive correlation $(\mathrm{r}=0.644, p<0.001)$ was found between FA score and depressive symptom severity. Total energy intake was not significantly different $(p=0.848)$ between MDD participants with and without FA. However, energy distribution was significantly different, saturated fat $(p=0.004)$ and trans fatty acids $(p=0.044)$ were higher, while polyunsaturated fats $(p=0.008)$ and carbohydrates $(p=0.025)$ were lower in MDD participants with versus without FA. This study provides novel evidence of a link between MDD, FA and dietary fats. This may help to explain the higher risk of chronic diseases and weight gain in MDD, warranting further investigation of the relationship and to determine causation.

\subsection{Orthorexia Nervosa Is Not More Prevalent among Athletes Compared to Healthy Controls: A Cross-Sectional Study}

\section{Mandy Foyster, Jessica Biesiekierski, Andrew Govus, Matilda Tonkovic, Nessmah Sultan and Caroline Tuck \\ Department of Nutrition and Dietetics, La Trobe University, Bundoora, Australia}

Orthorexia nervosa describes a pathological fixation with food based on its proposed health benefits and can lead to malnutrition and impaired performance. Athletes have a higher point prevalence of disordered eating compared to the general population; however, the prevalence of orthorexia in athletes in unknown. This cross-sectional study measured the prevalence of orthorexia among elite and recreational endurance athletes (runners, cyclists, triathletes who train $\geq 5 \mathrm{~h}$ /week) compared to healthy controls (HC). Via an online survey, orthorexia was assessed using two measures: the pneumonic 'Sick, Control, 
One-stone, Fat, Food' (SCOFF $\geq 2$ indicating disordered eating) and the Eating Habits Questionnaire (EHQ). Data were analysed using independent $t$-test (mean $\pm \mathrm{SD}$ ) or MannWhitney test (median \pm IQR). In total, 51 athletes and $53 \mathrm{HC}$ completed the study. There were no differences in orthorexic traits between groups when measured by the SCOFF (athlete vs. HC, respectively, $27 \pm 1.40$ vs. $0.962 \pm 1.27, p=0.939$ ) or EHQ (athlete vs. HC, respectively, $76.3 \pm 17.4$ vs. $73.5 \pm 16.7, p=0.849)$. Unlike other eating disorders, athletes may not be at greater risk of developing orthorexia compared to the general population. Future research with larger and more diverse samples is warranted.

\subsection{Executive Function Predicts Dietary Behaviour and Weight Status in Primary School Aged Children}

\section{Naomi Kakoschke and Ian Zajac \\ CSIRO, Adelaide, Australia}

Executive functioning (EF) plays a pivotal role in regulating human behaviours. To date, limited studies have examined the relationship between $\mathrm{EF}$ and dietary behaviour in young children, with most focused on single components of EF, and few examined both diet and weight status. We investigated the relationship between multiple EF components, diet, and weight status. Participants were $n=283$ Australian children aged 7-9 years. EF was assessed with the Behavior Rating Inventory of Executive Function (parent-reported) and cognitive performance (working memory, trails A\&B, digit symbol). Diet quality was assessed with the CSIRO Short Food Survey (parent-reported). Weight status was assessed with age-adjusted body mass index (BMI) controlling for age, sex, and muscle mass. Overweight children $(26 \%)$ consumed significantly more discretionary foods $(p=0.05)$ and meats $(p=0.048)$. Children with lower parent-reported EF capacity $(p=0.029)$ and cognitive performance $(p<0.001)$ consumed significantly more discretionary foods. Poorer cognitive performance also predicted overweight status $(p=0.009)$. The results demonstrate a link between EF and discretionary food intake, indicating lower diet quality in these children. These findings suggest that behavioural difficulties, which co-occur with low EF, may present challenges in relation to modifying discretionary food intake in children.

2.15. Cooking Confidence, Diet Quality, the Gut Microbiome, and Dietary and Gut Biomarkers of the Gut-Brain Axis

Joanna H. Rees ${ }^{1}$, Amanda Devine ${ }^{1}$, Claus T. Christophersen ${ }^{2}$, Johnny Lo ${ }^{3}$ and Shih Ching Fu ${ }^{1}$

1 Institute of Nutrition, School of Medical and Health Science, Edith Cowan University, Joondalup, Australia

2 Centre for Integrative Metabolomics \& Computational Biology, School of Science, Edith Cowan University, Joondalup, Australia

3 School of Science, Edith Cowan University, Joondalup, Australia

Poor diet quality, obesity and mental health disorders increase simultaneously with declining diet quality. Gut microbiome is affected by, and affects, all of these. Microbiomespecific dietary components positively affect 5-year mental health outcomes. Cooking interventions are a successful strategy for improving cooking confidence but may not change dietary behaviours. Method: This study explored the effects of a 7-week cooking program on cooking confidence, mental health, dietary and gut biomarkers, and the gut microbiome in Australian adults $(n=135)$. Results: Cooking confidence and perceived general health improved post-program. whilst no effect was observed for cooking confidence and diet quality, gut biomarkers, or microbiome composition. BMI was positively associated with faecal short-chain fatty acids (SCFA); poor diet quality was associated with reduced microbial diversity. Differences in microbial phylum distribution across BMI and diet quality categories reflected altered SCFA and bile acid metabolism associated with Westernised diets. Associations between dietary intake, body composition and metabolic 
pathways involved in gut-brain communications were seen. In a healthy cohort, the importance of diet quality has been reinforced; shifts in gut microbiome composition and gut biomarkers known to be detrimental to mental health were evident in those with poorer diet quality.

\title{
2.16. Molecular Characteristics of the Dose-Response Relationship of Dietary Lactoferrin
} Intervention on Neurodevelopment and Cognition in Piglets

\author{
Bing Wang ${ }^{1}$, Yue Chen ${ }^{2}$, Chanwei Yang ${ }^{2}$, Yujie Shi ${ }^{3}$, Zhizhong Dong ${ }^{4}$ and \\ Frederic A. Troy II ${ }^{5}$ \\ 1 Graham Centre for Agricultural Innovation, Charles Sturt University, \\ Wagga Wagga, Australia \\ 2 School of Medicine, Xiamen University, Xiamen, China \\ 3 Nestle Research Centre, Beijing, China \\ 4 Nutrition \& Health Research Institute, COFCO Corporation, Beijing, China \\ 5 Department of Biochemistry and Molecular Medicine, University of California School \\ of Medicine, Davis, USA
}

Lactoferrin (Lf), a sialic acid (Sia)-rich milk glycoprotein, can promote early neurodevelopment and cognition in neonatal piglets. The dose-dependency of Lf intervention remains unknown. The 3-day-old male piglets were randomly allocated to groups (Grp) 1 supplemented milk LF at $155 \mathrm{mg} / \mathrm{kg} /$ day (low dose) or $285 \mathrm{mg} / \mathrm{kg} /$ day (high dose, Grp 2) from postnatal day 3 to 38. Gene expression profiles and their cognate proteins were analysed. The rate of piglet learning and long-term memory was higher with the low dose, as assessed in an eight-arm radial maze. Global gene transcription profiling showed that the low dose upregulated genes and functions correlated with neurodevelopment and cognition, while the high dose showed a greater correlation with cellular processes modulating neuroprotection. The expression level of genes and proteins associated with Brain-derived neurotrophic factor (BDNF) was higher in both Lf groups and only low-doseactivated genes associated with BDNF signalling. Genes differentially expressed in the five highest networks showed that the low-dose was more strongly associated with cellular and neurocognitive development, while the high dose correlated with the prevention of neurodevelopmental disorders and neurological-associated diseases. The low dose of Lf intervention enhances neurodevelopmental and cognitive processes, while the high dose shows greater neuroprotection, findings of potential clinical relevance.

\subsection{Measured and Declared Vitamin D Concentrations in Australian Fortified Foods}

Katya M. Clark, Tony P. James, Hio Ian Ip, Eleanor Dunlop, Judy Cunningham and Lucinda J. Black

Curtin University, Perth, Australia

Fortified foods are an important source of vitamin D, since this nutrient occurs naturally in relatively low concentrations in a limited number of foods. We aimed to compare the measured and declared vitamin D concentrations in Australian fortified foods and assess compliance with regulatory requirements. Vitamin $\mathrm{D}_{3}, 25$-hydroxyvitamin $\mathrm{D}_{3}$, vitamin $\mathrm{D}_{2}$ and 25-hydroxyvitamin $\mathrm{D}_{2}$ were measured in 30 samples of 14 different food products (breakfast cereal, edible oil spread, malted chocolate drink powder and soy milk) using liquid chromatography with triple quadrupole mass spectrometry. Analytical concentrations ranged from half to nearly three times more than that declared on the nutrition information panel. Only 1 product had a measured vitamin D concentration close to the declared value, while 10 out of 14 products had measured vitamin D concentrations in excess of that declared. Two soy milks had analytical concentrations three times more than the maximum permitted amount, and one edible oil spread did not meet the minimum mandated vitamin D concentration. The nutrition information panel proved unreliable for assessing vitamin D concentration in fortified foods across all product categories, which 
has implications for estimation of vitamin $\mathrm{D}$ intakes. Tolerance limits for manufacturers may be required to improve confidence in the declared concentrations.

\subsection{Relationship between Energy Intake and Tissue Deposition in Pigs with Modern Genetics}

Fan Liu ${ }^{1}$, Chris Brewster ${ }^{1}$, Samantha Gilmour ${ }^{1}$, David Henman ${ }^{1}$, Robert Smits ${ }^{2}$, Frank Dunshea ${ }^{3,4}$, Brian Luxford ${ }^{1}$, John Pluske ${ }^{3,5}$ and Roger Campbell ${ }^{6}$

1 Rivalea Australia, Corowa, Australia

2 Australian Pork Limited, Barton, Australia

3 The University of Melbourne, Parkville, Australia

4 University of Leeds, Leeds, UK

5 Australasian Institute of Pig Research Ltd., Willaston, Australia

6 RG Campbell Advisory Pty Ltd., Semaphore, Australia

Pigs have been selected for low backfat for decades to suit the Australian market. Restricting energy intake below the maximum protein deposition is used by animal nutritionists to control carcass fatness. It is unknown whether the fatness is responsive to energy levels in the pigs selected for low backfat; thus, this study quantified the relationship between energy intake and tissue deposition. Int toal, 56 male and 56 female pigs (Primegro Genetics, Corowa, NSW, Australia) were fed 7 levels of a wheat-based diet (ranging from 26 to $44 \mathrm{MJ}$ digestible energy (DE)/day) between 60 and $108 \mathrm{~kg}$ weight. The body composition of anaesthetised pigs was measured using Dual Energy X-ray Absorptiometry at $108 \mathrm{~kg}$. Backfat increased by $0.13 \mathrm{~mm}$ for every MJ increase in daily DE intake in males $(p=0.004)$ but was not affected in female pigs. The fat composition and deposition rate both increased linearly in males but increased at a reduced rate in females in response to DE intake (all $p<0.01$ ). Protein deposition rate increased linearly with DE intake in both sexes (both $p<0.001$ ). In conclusion, the pigs, particularly the females, that were selected for low backfat had a blunted response of backfat to energy intake.

\subsection{Freeze-Thaw Effect on Anthocyanin Reference Standards}

\section{Mamatha Chandra Singh ${ }^{1}$, Celine Kelso ${ }^{2}$, William E. Price ${ }^{3}$ and Yasmine Probst ${ }^{1}$}

1 School of Medicine, Illawarra Health and Medical Research Institute, Faculty of Science, Medicine and Health, University of Wollongong, Wollongong, Australia

2 School of Chemistry and Molecular Bioscience, Illawarra Health and Medical Research Institute, Molecular Horizons Institute, Faculty of Science, Medicine and Health, University of Wollongong, Wollongong, Australia

3 School of Chemistry, Faculty of Science, Medicine and Health, University of Wollongong, Wollongong, Australia

Anthocyanins, a class of flavonoids in fruit and vegetables, have several health benefits. Nevertheless, they are very unstable and highly reactive compounds. The precision in the anthocyanin quantification is dependent on the purity and the stability of the reference standards that are obtained by extraction (from natural sources in the laboratory or commercially available standards). Additionally, the quantification of anthocyanin compounds in samples depend on the calibration curves that can be created from the reference standards. Across reported studies, reference standards were extracted, purified, and or prepared in various solvents/solvent compositions and stored at temperatures ranging from 4 to $-80{ }^{\circ} \mathrm{C}$. This study was therefore designed to determine the stability of anthocyanin reference standards. The anthocyanin-3-glucoside reference standards ( $5 \mu \mathrm{g} / \mathrm{mL}$ to $75 \mu \mathrm{g} / \mathrm{mL})$ were prepared, stored at $-80{ }^{\circ} \mathrm{C}$ and freeze-thawed for 3 months. The degradation of each standard was monitored using a liquid chromatography-mass spectrometer. A significant decrease $(p$-value $<0.00001)$ in the anthocyanin concentration was observed after each freeze/thaw cycle in a short time. Despite all standards being freeze-thawed at the same time, a difference in the anthocyanin degradation pattern was observed. Therefore, for precision in anthocyanin quantification, it is important to understand the factors that govern the stability of reference standards. 
2.20. A Comparison of Game Day Energy and Carbohydrate Intake to Recommendations in Women's Australian Football League Athletes

Chloe Otte ${ }^{1}$, Evangeline Mantzioris ${ }^{1,2}$, Brianna Salagaras ${ }^{3}$ and Alison Hill 1,2

1 Clinical and Health Sciences, University of South Australia, Adelaide, Australia

2 Alliance for Research in Exercise, Nutrition and Activity (ARENA), University of South Australia, Adelaide, Australia

3 Adelaide Football Club, Adelaide, Australia

Carbohydrate intake is essential for sports performance and recovery. This study aimed to capture game day dietary intakes of women's Australian Football League (AFL) athletes to determine if they meet daily and periodised carbohydrate $(\mathrm{CHO})$ recommendations established by The American College of Sports Medicine. A combination of self-report, direct observation (video footage), and fluid analysis (bottle weighing) were used to determine dietary intake of 17 women's AFL athletes (age $25 \pm 4.5$ years; weight $66 \pm 7 \mathrm{~kg}$ ) on 5 home game days. Daily energy requirements were met by $71 \%$ of athletes; however only, $18 \%$ met the minimum daily $\mathrm{CHO}$ recommendations (intake $(\mathrm{I}), 4.9 \mathrm{~g} / \mathrm{kg} /$ day vs. recommendations $(\mathrm{R}), 6 \mathrm{~g} / \mathrm{kg} /$ day, $p=0.001)$. Less than $20 \%$ of athletes met pre-game recommendations ( $\mathrm{I}, 1 \mathrm{~g} / \mathrm{kg} /$ day vs. $\mathrm{R}, 3 \mathrm{~g} / \mathrm{kg} /$ day, $p=0.000$ ), and less than $50 \%$ consumed sufficient $\mathrm{CHO}$ during games (I, $23 \mathrm{~g}$ vs. $\mathrm{R}, 60 \mathrm{~g}, p=0.029$ ). All athletes met post-game recovery recommendations. This is the first study to evaluate game day intake in professional women's AFL athletes. Overall, these athletes failed to meet daily and periodised $\mathrm{CHO}$ recommendations. These findings will help guide strategies to improve athletic performance and reduce potential health risks associated with inadequate intake in female athletes.

2.21. Obesity Increases Airway Inflammation in Adults with Asthma: A Systematic Review and Meta-Analysis

Hayley A. Scott ${ }^{1,2}$, Shawn Hong Ming Ng ${ }^{3}$, Rebecca F. McLoughlin 1,4, Sarah R. Valkenborghs ${ }^{2,5}$ and Lisa G. Wood ${ }^{1,2}$

1 Priority Research Centre for Healthy Lungs, The University of Newcastle, Newcastle, Australia

2 School of Biomedical Sciences and Pharmacy, The University of Newcastle, Newcastle, Australia

3 School of Medicine and Public Health, The University of Newcastle,

Newcastle, Australia

4 School of Nursing and Midwifery, The University of Newcastle, Newcastle, Australia

5 Priority Research Centre for Physical Activity and Nutrition, The University of

Newcastle, Newcastle, Australia

Obesity is associated with more severe asthma; however, the mechanisms responsible are not understood. This review sought to examine whether obesity is associated with increased airway inflammation in asthma. A systematic search was completed in Medline, Embase, CINAHL, Scopus and Current Contents up to August 2021. Studies reporting airway inflammation in obese versus non-obese adults with asthma were assessed for inclusion. Thirty-two studies were included. Obese asthmatics had higher sputum neutrophils than non-obese asthmatics (MD $=5.0 \%, 95 \% \mathrm{CI}: 1.2$ to $8.9 ; p=0.01, I^{2}=42 \%$ ). While there was no difference in sputum eosinophils, obese asthmatics had higher bronchial submucosal eosinophils (SMD $=0.58,95 \%$ CI: 0.25 to $0.91 ; p<0.01, I^{2}=0 \%$ ). Sputum IL-5 was also increased in obesity ( $\mathrm{MD}=0.90 \mathrm{pg} / \mathrm{mL}, 95 \% \mathrm{CI}: 0.24$ to $1.56 ; p<0.01, I^{2}=0 \%$ ). This review found that obesity is associated with a different pattern of airway inflammation in adults with asthma. Neutrophilic inflammation is increased in the sputum of obese asthmatics, while eosinophilic inflammation is increased in the bronchial submucosa. Sputum IL-5, a key mediator in eosinophil activation, is also increased in obesity. These findings may explain the increased asthma severity observed in obesity. 
2.22. Effect of 14-Day Oral Soluble Fibre Interventions Using Different Dosage Strategies, in Adults with Asthma-A Randomised Controlled Trial

\author{
Bronwyn S. Berthon ${ }^{1}$, Lily M. Williams ${ }^{1}$, Netsanet A. Negewo ${ }^{1}$, \\ Cherry A. Thompson ${ }^{1}$, Rebecca F. McLoughlin ${ }^{1}$, Peter A.B. Wark ${ }^{1,2}$ and \\ Lisa G. Wood ${ }^{1}$ \\ 1 Priority Research Centre for Healthy Lungs, Hunter Medical Research Institute, \\ University of Newcastle, Newcastle, Australia \\ 2 Respiratory and Sleep Medicine, John Hunter Hospital, Newcastle, Australia
}

Soluble fibre interventions have been shown to modulate airway inflammation and asthma control in both experimental studies and subjects with asthma. This study investigated the effect of different soluble fibre dosing interventions on asthma control, airway inflammation, and plasma short-chain fatty acids (SCFAs). A randomised, double-blind, placebo-controlled 4-way crossover trial in 32 subjects with stable, not-well controlled asthma was performed. Subjects received $4 \times 14$-day, oral oligosaccharide blend interventions in random order: $6 \mathrm{~g}$ twice daily (HD2 $\times 6$ ), $12 \mathrm{~g}$ once daily (HD12), $6 \mathrm{~g}$ once daily (LD), and placebo, with 2-week washouts. Clinical assessment before and after each intervention included: asthma control (ACQ-7), asthma-related quality of life (AQLQ), lung function, sputum cell counts, plasma SCFA, and full blood count. Following LD, ACQ7 improved, and sputum and blood eosinophil counts decreased compared to baseline. HD12 increased total plasma SCFA and acetate concentrations compared to baseline and placebo, and improved AQLQ compared to baseline. No significant changes in lung function were observed. In conclusion, modest improvements in asthma control, asthma quality of life and inflammation were observed following soluble fibre supplementation using an oligosaccharide blend. Further studies are needed to determine the therapeutic potential of soluble fibre in asthma.

\title{
2.23. Soluble Fibre Supplementation in Subjects Asthma: A Laboratory Analysis of an RCT
}

Lily M. Williams 1,2 , Bronwyn S. Berthon 1,2, Rebecca F. McLoughlin 1,3, Netsanet A. Negewo ${ }^{1,4}$, Cherry A. Thompson ${ }^{1,2}$, Peter A.B. Wark ${ }^{1,4,5}$ and Lisa G. Wood ${ }^{1,2}$

1 Priority Research Centre for Healthy Lungs, Hunter Medical Research Institute, New Lambton Heights, Australia

2 School of Biomedical Sciences and Pharmacy, University of Newcastle, Callaghan, Australia

3 School of Nursing and Midwifery, The University of Newcastle, Callaghan, Australia

4 School of Medicine and Public Health, The University of Newcastle,

Callaghan, Australia

5 Department of Respiratory and Sleep Medicine, John Hunter Hospital, Hunter New England Health, New Lambton Heights, Australia

Soluble fibre has been shown to modulate inflammation in asthma. This study examined the effect of 14-day low- (LD, $6 \mathrm{~g} /$ day) and high-dose (HD, $12 \mathrm{~g} /$ day) soluble fibre supplementation in adults $(n=20)$ with asthma on sputum and peripheral blood mononuclear cells (PBMCs) gene expression, and on inflammatory cytokine release by PBMCs. IL-4, -5 , and -13 and HDAC mRNA expression were measured via RT-qPCR. PBMCs were cultured with $5 \mathrm{ng} / \mathrm{mL}$ lipopolysaccharide (LPS) or $20 \mathrm{MOI}$ rhinovirus 1B (RV1B/Newcastle/2018) for 48 h. TNF- $\alpha$, IFN- $\gamma$, and IL-10 were measured by ELISA. Following LD, PBMC HDAC1 expression was upregulated compared to baseline $(p=0.043)$. There were no differences in LPS or RV1B-induced TNF- $\alpha$ or IL-10, though RV1B-induced IFN- $\gamma$ decreased following HD compared to baseline $(p=0.010)$. Subgroup analysis showed in subjects with noneosinophilic airway inflammation (sputum eosinophils $<3 \%, n=12$ ), there was a decrease in LPS-induced TNF- $\alpha$ following HD compared to LD $(p=0.017)$, while LPS-induced IL-10 increased following HD compared to baseline $(p=0.049)$. Also, 
RV1B-induced TNF- $\alpha(p=0.010)$, IL-10 $(p=0.017)$, and IFN- $\gamma(p=0.028)$ decreased compared to baseline. These observations demonstrate soluble fibre modulates inflammatory responses in PBMCs from asthma subjects with noneosinophilic inflammation.

2.24. Krill Oil Improved Osteoarthritic Knee Pain in Adults with Mild to Moderate Osteoarthritis of the Knee: A 6-Month Multicentre, Randomised Double-Blind, Placebo-Controlled Trial

\section{Welma Stonehouse ${ }^{1}$, Bianca Benassi-Evans ${ }^{1}$, Jana Bednarz ${ }^{2}$, Andrew Vincent ${ }^{3}$, Stephen Hall ${ }^{4}$ and Catherine L. Hill ${ }^{5}$ \\ 1 CSIRO, Adelaide, Australia \\ 2 Adelaide Health Technology Assessment (AHTA), University of Adelaide, \\ Adelaide, Australia \\ 3 Freemasons Centre for Male Health \& Wellbeing, University of Adelaide, Adelaide, Australia \\ 4 Emeritus Research Pty Ltd., Monash University, Melbourne, Australia \\ 5 Rheumatology Unit, The Queen Elizabeth and Royal Adelaide Hospitals, Adelaide, Australia}

Krill oil, rich in anti-inflammatory long-chain (LC) omega-3 polyunsaturated fatty acids (PUFA) and astaxanthin, may be an effective treatment for osteoarthritis (OA) symptoms. The effects of Swisse High Strength Deep Sea Krill oil (Superba ${ }^{\mathrm{TM}}$ BOOST) (KO) on knee pain in adults with mild to moderate knee OA were investigated in a 6-month double-blind, randomised, placebo-controlled, multicentre trial. Healthy adults $(n=235)$, 40-65 yrs, BMI $>18.5-<35 \mathrm{~kg} / \mathrm{m}^{2}$, clinically diagnosed with mild to moderate knee OA regular knee pain and consuming $<0.5 \mathrm{~g}$ LC omega-3 PUFA/day were randomised to either $4 \mathrm{~g} \mathrm{KO} /$ day (0.88 g LC omega-3 PUFA/day, $0.45 \mathrm{~g} /$ day astaxanthin) or placebo (mixed vegetable oil) groups. Knee outcomes were assessed at baseline, 3 and 6 months using the Western Ontario and McMaster Universities Arthritis Index (WOMAC) numeric scale. Omega-3 Index (marker of LC omega-3 PUFA status) increased with KO vs. placebo (6.0\% to $8.9 \%$ vs. $5.5 \%$ to $5.4 \%$, respectively, $p<0.001$ ). Knee pain score improved more with $\mathrm{KO}$ vs. placebo (adjusted mean difference [95\% CI] in change from baseline between groups at 6 months: $-5.2[-10.0,-0.3], p=0.04)$. Knee stiffness and physical function were similarly improved $(p<0.05)$. Overall, $\mathrm{KO}$ resulted in modest improvements in knee outcomes in adults with mild to moderate knee OA.

2.25. The Associations of Butyrate-Producing Bacteria of the Gut Microbiome with Diet Quality and Muscle Health

Jessica A. Davis ${ }^{1}$, Fiona Collier ${ }^{2,3}$, Mohammadreza Mohebbi ${ }^{4}$, Julie A. Pasco ${ }^{1,2,5,6}$, Nitin Shivappa ${ }^{7,8}$, James R. Hebert ${ }^{7,8}$, Felice N. Jacka ${ }^{1,9,10,11}$ and Amy Loughman ${ }^{1}$

1 Deakin University, IMPACT-The Institute for Mental and Physical Health and Clinical Translation, School of Medicine, Barwon Health, Geelong, Australia

2 Barwon Health, Geelong, Australia

3 Geelong Centre for Emerging Infectious Diseases (GCEID), Barwon Health, Geelong, Australia

4 Faculty of Health, Biostatistics Unit, Deakin University, Geelong, Australia

5 Department of Medicine-Western Health, The University of Melbourne, St Albans, Australia

6 Department of Epidemiology and Preventive Medicine, Monash University, Prahran, Australia

7 Cancer Prevention and Control Program, University of South Carolina, Columbia, USA

8 Department of Epidemiology and Biostatistics, Arnold School of Public Health, University of South Carolina, Columbia, USA

9 Centre for Adolescent Health, Murdoch Children's Research Institute, Melbourne, Australia

10 James Cook University, Townsville, Australia

11 Black Dog Institute, Australia 
This study aimed to investigate the relationships between diet quality, the relative abundance of butyrate-producing bacteria of the gut microbiome, and muscle mass, strength, and function. In this cross-sectional study, $n=490$ men $(64.4 \pm 13.5$ years) from the Geelong Osteoporosis Study provided food frequency questionnaire data, from which the Australian Recommended Food Score (ARFS) and Dietary Inflammatory Index $\left(\mathrm{DII}^{\odot}\right)$ score were calculated. Muscle mass (skeletal muscle index from DXA-derived lean mass), muscle strength (handgrip strength), and muscle function (Timed Up-and-Go test) were measured. Participants provided stool samples for 16S rRNA gene sequencing. There was no evidence of associations between alpha or beta diversity and muscle health measures. A healthier ARFS score was positively associated with the relative abundance of butyrate-producing bacteria ( $\beta 0.09,95 \%$ CI: $0.03,0.15)$, and a higher (pro-inflammatory) DII score was associated with lower relative abundance of butyrate-producing bacteria $(\beta-0.60,95 \% \mathrm{CI}:-1.06,-0.15)$. The relative abundance of butyrate-producing bacteria was positively associated with healthier muscle mass, strength, and function; however, these relationships were attenuated in multivariable models. These findings support the role of diet quality in achieving a healthier gut microbiome, however, further evidence is required for a gut-muscle axis in humans.

2.26. Maternal Pre-and Perinatal Influences on Secular Birthweight Trends in Tasmania: A Retrospective Analysis of All Live Singleton Births 2005-2018

\section{Manoja P. Herath, Andrew P. Hills, Jeffrey M. Beckett, Sisitha Jayasinghe, Nuala M. Byrne and Kiran D.K. Ahuja School of Health Sciences, University of Tasmania, Launceston, Australia}

Tasmania has the highest rates of child and adult obesity in Australia. Birthweight, a key indicator of foetal nutrition in utero, is a crucial determinant of obesity in childhood, adolescence and adulthood. We explored birthweight trends and associations with maternal characteristics in live-born infants between 2005 and 2018 using regression analysis. The Tasmanian Data Linkage Unit provided the matched and deidentified perinatal and birthweight data of 81700 mother-infant pairs. While the mean birthweight (3425 $\mathrm{g}$ to $3359 \mathrm{~g})$ and the proportion of high birthweight $(14.2 \%$ to $11.0 \%)$ decreased, there was an increase in the proportion of low birthweight neonates (4.8\% to 6.5\%) in the 14-year period. A downward shift in gestation length distribution, increased rates of caesarean delivery, hypertensive disorders, age $>35$ years, assisted conception, and changes in ethnic composition with increased numbers of indigenous mothers and mothers born outside of Australia partly contributed to this trend. Interestingly, the impact of striking increases in the rates of pre-pregnancy obesity and gestational diabetes mellitus, factors commonly associated with increased birthweight, were not observed in this study. Future studies should consider other maternal factors potentially contributing to these trends, including gestational weight gain and glycaemic control in diabetic mothers.

\subsection{Breastfeeding Patterns in a Cohort of Indigenous Australian Children}

\section{Oyepeju Onifade, Tracy Schumacher, Megan Rollo, Kym Rae and Kirsty Pringle}

1 School of Medicine and Public Health, University of Newcastle, Tamworth, Australia

2 Department of Rural Health, School of Medicine and Public Health, Faculty of Health and Medicine, University of Newcastle, Tamworth, Australia

3 School of Health Sciences, University of Newcastle, Callaghan, Australia

4 Mater Research Institute, South Brisbane, Australia

5 School of Biomedicine and Pharmacy, University of Newcastle, Callaghan, Australia

Breastfeeding is an important component of early childhood nutrition. Breastmilk, the recommended first food of the infant, is suited to exclusively meet the infant's nutrient requirement for adequate growth in the first 6 months and with food supplementation for up to 2 years of age. The aim of this study was to determine the pattern of breastfeeding among Indigenous infants in the Gomeroi gaaynggal cohort. Children attended up to 
four visits in the first year (3, 6, 9 and 12 months) and once a year afterwards. Child breastfeeding details were obtained by a qualified dietitian via interviewer-administered dietary surveys. Intention to breastfeed details were obtained from the mothers' hospital antenatal records. Descriptive statistics were conducted using Stata 15/IC. A total of 305 breastfeeding data was obtained from 133 individual infants. At 3 months, 36\% of the infants were being breastfed although $89 \%$ were breastfed on arrival at home after delivery. During pregnancy, $72 \%$ of the mothers indicated intention to breastfeed. Findings from this study showed a high rate of breastfeeding with a high rate of decline at 3 months. Future studies should investigate factors influencing the breastfeeding practice among this population.

\title{
2.28. Bidirectional Associations between Parental Feeding Practices, Infant Appetitive Traits and
} Infant Weight

\author{
Alissa J Burnett ${ }^{1}$, Elena Jansen ${ }^{2}$, Jessica Appleton ${ }^{3}$, Christine Rossiter ${ }^{4}$, \\ Cathrine Fowler ${ }^{3}$, Elizabeth Denney-Wilson ${ }^{3}$ and Georgie Russell ${ }^{1}$ \\ 1 School of Exercise and Nutrition Sciences, Deakin University, Geelong, Australia \\ 2 School of Medicine, Johns Hopkins University, Baltimore, USA \\ 3 Faculty of Medicine and Health, The University of Sydney, Sydney, Australia \\ 4 Faculty of Health, University of Technology Sydney, Sydney, Australia
}

Little is known about the pathways linking parent feeding practices with appetitive traits and weight throughout infancy. This study examined cross-lagged associations between parental feeding practices, infant appetitive traits, and infant BMI. Parents $(n=380)$ of infants aged less than 6 months at baseline reported their feeding practices, infant appetitive traits, and infant weight at three timepoints up to 12 months of age. Cross-lagged models examined the bidirectional associations between parent feeding practices, infant appetitive traits, and infant BMI. There was strong continuity across the three timepoints for maternal feeding practices, infant appetitive traits, and infant BMI. Infant food avoidance was prospectively associated with higher parental persuasive feeding. Infant BMI was prospectively associated with higher parent-led feeding. Infant food approach was prospectively associated with higher infant BMI, and infant BMI was prospectively associated with higher food approach. Feeding on demand was prospectively associated with lower infant food approach. This study highlights the complex inter-relationships between parental feeding practices, infant appetitive traits, and infant weight. Most of the significant associations originated with the infant, indicating that training should be provided to parents in future interventions to learn how to respond to infant appetitive traits and weight.

2.29. Outcomes of the Time for Healthy Habits Trial: Translation of Two Healthy Eating and Active Living Programs for Parents of 2- to 6-Year-Old Children

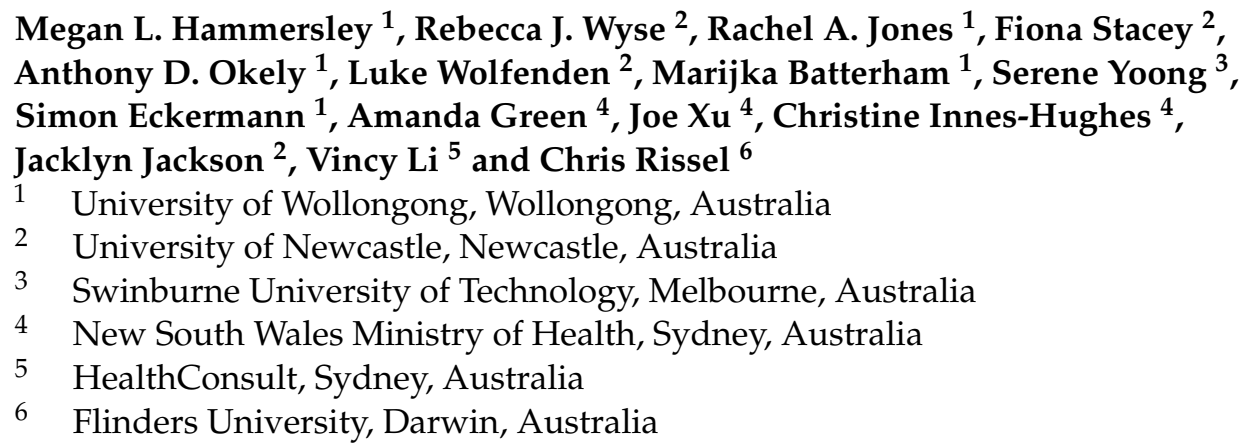

The Time for Healthy Habits translation study assessed the effectiveness of online and telephone interventions in improving child fruit and vegetable intake, non-core food intake, body mass index (BMI), and movement behaviours. This partially randomised preference trial design included 458 parents of 2- to 6-year-old children. The telephone intervention 
(Healthy Habits Plus) comprised six calls, the online intervention (Time2bHealthy) comprised six modules, and the active control comprised information sheets. A telephone questionnaire was used at baseline and 9 months post-baseline to collect data from parents. Data were analysed for randomised participants only $(n=240)$, preference participants only $(n=218)$, and all participants in the trial $(n=458)$. When comparing the telephone and online interventions to the control, there was no significant improvement in fruit and vegetable intake (primary outcome). There was a significant improvement in non-core food intake for the telephone intervention compared to the control $(p<0.001)$ in the randomised trial only and analyses of all participants combined. Differences between the interventions for other outcomes were small. In summary, compared with written materials, the telephone and online interventions did not improve child fruit and vegetable intake; however, there were improvements in non-core food intake for the telephone intervention.

\subsection{Combined Protein and Exercise Interventions to Improve Muscle Mass, Strength and} Function in Older Adults: A Systematic Review

\section{Isobel L. Stoodley ${ }^{1,2}$, Lily M. Williams ${ }^{1,2}$, Bronwyn S. Berthon ${ }^{1,2}$, Hayley A. Scott ${ }^{1,2}$ and Lisa G. Wood ${ }^{1,2}$ \\ 1 School of Biomedical Science and Pharmacy, University of Newcastle, Newcastle, Australia \\ 2 Priority Research Centre for Healthy Lungs, Hunter Medical Research Institute, Newcastle, Australia}

Age-related loss of muscle mass, strength and function is associated with poorer health outcomes. Interventions targeting exercise and protein intake are important for prevention and treatment, however, evidence supporting combining these interventions is limited. This systematic review summarised the evidence for protein + exercise versus exercise alone on outcomes of muscle mass, strength and function in adults $\geq 65$ years. English language articles up to May 2020 were identified via MEDLINE, EMBASE, CINAHL and Cochrane databases. In total, 26 studies ( $n=2720$, median duration 12 weeks) were included, with varying protein types (65\% milk-related products), doses (median $15 \mathrm{~g}$, range 3-60 g/day) and frequencies (81\% daily, 19\% after exercise only). Additional benefits of protein + exercise versus exercise alone were found for muscle mass gain ( 5 of 18 studies) and knee extension strength ( 3 of 17 studies) [SMD $=0.17(0.03,0.31), p=0.02]$, but not timed-upand-go or handgrip strength. Protein with meals was superior for building muscle, while between meals was beneficial for knee extension strength. Supplementing daily (versus exercise days only) was superior for fat loss, muscle gain and knee extension strength. Combining protein with exercise is beneficial, however, more research is needed into ideal type and dose of protein for improvements in muscle mass, strength and function.

\subsection{Novel Gas Capsule Demonstrates Promotion of Distal Fermentation When a Low Fodmap Diet Is Supplemented with Dietary Fibres in Irritable Bowel Syndrome}

\section{Daniel So ${ }^{1}$, Chu K. Yao ${ }^{1}$, Paul A Gill ${ }^{1}$, Zaid S. Ardalan ${ }^{1}$, Phoebe A. Thwaites ${ }^{1}$, Kyle J. Berean ${ }^{2}$, Jane G. Muir ${ }^{1}$ and Peter R. Gibson ${ }^{1}$ \\ 1 Gastroenterology, Monash University and Alfred Hospital, Melbourne \\ 2 Atmo Biosciences, Melbourne}

A low FODMAP diet (LFD) in patients with irritable bowel syndrome (IBS) prevents excessive fermentation in the proximal colon, but may be associated with diminished distal colonic fermentation. We aimed to determine if the co-supplementation of poorly fermented with fermentable fibre pushes fermentation distally when initiating an LFD in patients with IBS. In a double-blind feeding study, patients were randomly allocated to one of three diets: 'Control' (LFD); 'Sugarcane' (LFD with poorly fermented bagasse); 'Combination' (LFD with bagasse and fermentable-resistant starch) and crossed over twice to the other diets. Short-chain fatty acids (SCFA) were measured in plasma and faeces, and luminal colonic hydrogen concentrations were measured telemetrically via gas-sensing capsule. In 
20 patients, the Combination diet was associated with greater colonic fermentation, with $\sim 64 \%$ higher plasma SCFA compared with Control diet $(p=0.028)$ but not reflected in faecal SCFA across the diets. Colonic hydrogen concentrations fell distally with the Control diet to $1.7 \%$ but were more than 2 -fold greater with the Combination diet $(3.5 \% ; n=12 ; p=0.037)$. In contrast to the LFD alone, the supplementation of poorly fermented with fermentable fibre is associated with evenly distributed fermentation around the colon. The novel power of the gas-sensing capsule in defining colonic luminal physiology is demonstrated.

\subsection{The Relationship between Dietary Factors and Functional Dyspepsia Symptoms: A Cross-Sectional Study}

\section{Zoe Cooke, Stephanie Resciniti, Caroline Tuck and Jessica Biesiekierski Department of Dietetics, Nutrition, and Sport, La Trobe University, Bundoora, Australia}

Functional dyspepsia (FD) affects $20 \%$ of the population and is characterised by early satiety, postprandial fullness, epigastric pain and/or burning without organic disease. Dietary management guidelines are lacking, and current approaches may be nutritionally imbalanced. This study aimed to measure associations between nutritional intake, dietary behaviours, and quality of life in those with FD and healthy volunteers (HV). Participants with FD $(n=115)$ and HV $(n=55)$ completed a cross-sectional, online survey including the Depression Anxiety and Stress Scale and food frequency questionnaire. Analysis was conducted using chi-square and Student's $t$-test. Fermentable carbohydrates (FODMAPs) $(56.1 \%)$, fatty foods (46.4\%), chilli $(43.8 \%)$ and wheat $(41.2 \%)$ were identified as triggers in FD. Adherence to special diets was greater in FD (78\%) compared to HV $(45 \% ; p=<0.001)$. Dietary advice was provided by a dietitian in $51.8 \%$ of FD participants and self-initiated in $21.5 \%$. FD had a greater prevalence of anxiety $(42.6 \%)$ and depression $(30.4 \%)$ compared to HV $(19.2 \%$ and $15.4 \%$, respectively, $p=0.03$ and $p=0.003)$. FD participants follow special diets and identify perceived trigger foods, with many reporting FODMAPs as a key trigger. Randomised controlled trials are needed to investigate effective dietary management approaches for FD.

\subsection{Associations between Lifestyle, Genetic Risk and Incidence of 13 Cancers: A Prospective Cohort Study}

Stephanie Byrne ${ }^{1,2}$, Terry Boyle ${ }^{1,2}$, Beben Benyamin ${ }^{1,2}$, S. Hong Lee ${ }^{1,2}$, Muktar Ahmed ${ }^{1,3}$ and Elina Hypponen 1,3

1 Australian Centre for Precision Health, University of South Australia,

Adelaide, Australia

2 UniSA Allied Health \& Human Performance, University of South Australia, Adelaide, Australia

3 UniSA Clinical and Health Sciences, University of South Australia, Adelaide, Australia

We examined associations between lifestyle, genetic risk and cancer incidence for 13 cancer types using prospective cohort data from the UK Biobank. Between 2006 and 2010, participants completed a questionnaire, had anthropometric measures taken, provided a blood sample, and were followed up until 2015 to 2016. Our analyses were restricted to those of white European ancestry with no history of malignant cancer $(n=196,485$, $50 \%$ female, aged 37-73 years). For each participant, a healthy lifestyle index (HLI) was computed based on cancer prevention recommendations for body weight, diet, alcohol, smoking, and physical activity, and a polygenic risk score (PRS) was calculated for each cancer, representing genetic risk. We used cox regression to examine the relationships between HLI, PRS, and cancer incidences. We found, for all cancer types, those in the top PRS tertile had an increased cancer risk compared to those in the bottom tertile, or there was a trend in that direction. Those with an unfavourable lifestyle had a higher risk of cancer for eight cancer types including pancreatic (HR [95\%CI] 2.06 [1.47,2.91]), bladder (1.95 [1.43,2.66]), kidney $(1.90$ [1.43,2.54]), and lung cancers $(3.41[2.76,4.20])$. These relationships did not differ by genetic risk, suggesting a healthy lifestyle may reduce risk of several cancers, even in those with a high genetic risk. 
2.34. Higher Consumption of Ultra-Processed Foods Is Associated with Decreased Time to Relapse in Multiple Sclerosis

Adrienne Misson ${ }^{1}$, Madeleine Stephens ${ }^{1}$, Alison Daly ${ }^{1}$, Robyn Lucas ${ }^{2,3}$,

Anne-Louise Ponsonby ${ }^{4,5}$, Ingrid van der $\mathrm{Mei}^{6}{ }^{6}$, Bruce Taylor ${ }^{6}$,

Ausimmune AusLong ${ }^{7}$ and Lucinda Black ${ }^{1}$

1 Curtin School of Population Health, Curtin University, Perth, Australia

2 Centre for Ophthalmology and Visual Science, The University of Western Australia, Perth, Australia

3 National Centre for Epidemiology and Population Health, Research School of Population Health, Australian National University, Canberra, Australia

4 Florey Institute of Neuroscience and Mental Health, The University of Melbourne, Melbourne, Australia

5 Murdoch Children's Research Institute, The University of Melbourne, Melbourne, Australia

6 Menzies Institute for Medical Research, University of Tasmania, Hobart, Australia

7 Investigator Group

The consumption of ultra-processed foods is associated with adverse health outcomes; however, the association with multiple sclerosis (MS) progression is unknown. We aimed to test the association between the consumption of ultra-processed foods and time to relapse in people with MS. We used data from the AusLong Study, a population-based study of people with a first clinical diagnosis of central nervous system demyelination at baseline, with reviews at 5 and 10 years. Habitual dietary intake was collected longitudinally using a food frequency questionnaire. By summing the intakes of 28 foods/beverages identified as ultraprocessed, we calculated energy-adjusted daily servings of ultra-processed foods. We used Cox proportional hazards regression to model time to relapse predicted by consumption of ultra-processed foods. The model included 775 events (115 of 274 participants had relapses). Covariate interactions with consumption of ultra-processed foods were tested; statistically significant $(p<0.1)$ covariates were retained in the final model. Higher consumption of ultraprocessed foods (per one energy-adjusted serving/day) was significantly associated with decreased time to relapse (adjusted hazard ratio $=1.006$; $95 \%$ CI: 1.002, 1.010), translating to a $22 \%$ decrease in time to relapse per quintile increase in the consumption of ultra-processed foods. Limiting the consumption of ultra-processed foods may have a protective effect on time to relapse.

\subsection{An Analysis on the Implementation of the Evidence-Based PCOS Lifestyle Guideline:} Recommendations from Women with PCOS

\section{Siew Lim ${ }^{1}$, Breanna Wright ${ }^{2}$, Melissa Savaglio ${ }^{1}$, Denise Goodwin ${ }^{2}$, Stephanie Pirotta ${ }^{1}$ and Lisa Moran ${ }^{1}$ \\ 1 Monash Centre for Health Research and Implementation, Clayton, Australia \\ 2 BehaviourWorks Australia, Monash Sustainable Development Institute, Monash University, Melbourne, Australia}

Polycystic ovary syndrome (PCOS) is the most common endocrinological disorder affecting women of reproductive age, affecting $8-13 \%$ of women in this group. Women with PCOS are more likely to have excess BMI, which in turn exacerbates the symptoms of PCOS in these women. The latest evidence-based guideline recommends lifestyle management as the first-line treatment for PCOS. However, the implementation of this recommendation through health services faces a significant challenge. As part of the mapping of the implementation plan for lifestyle management in PCOS, citizen panels and semi-structured interviews were conducted to capture the voices of consumers. Women with PCOS expressed the need for multidisciplinary, integrated care as a recurrent theme. Other important considerations included health professionals who listen and are open to learning about PCOS, the empowerment of women to self-manage PCOS and the provision of peer support. Women with PCOS also expressed the key recommendation of focussing 
on practical skills when providing lifestyle advice. Within that, both individual and group lifestyle sessions were valued for privacy and peer support, respectively, and delivery by a dietitian is preferred. These recommendations by women with PCOS should be considered when developing the implementation plan for the PCOS lifestyle guideline.

\title{
2.36. Exploring the Role of the Dietitian in the Management of Patients Admitted to Hospital for Alcohol Withdrawal

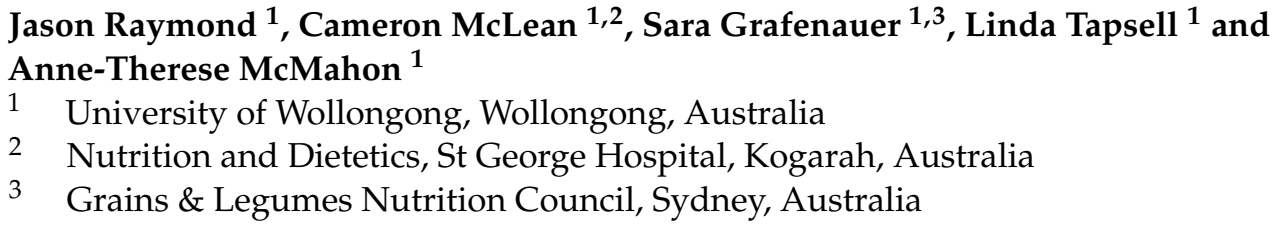

Evidence-based guidelines provide recommendations on nutritional considerations and emphasise multidisciplinary team (MDT) management of patients undergoing alcohol withdrawal. However how this is best achieved is unclear. This study aimed to explore how the MDT perceives the dietitian's role in the nutritional management of patients admitted to hospital for alcohol withdrawal. Allied health professionals including dietitians, speech pathologists, social workers, occupational therapists, and physiotherapists $(n=22)$ were recruited from a tertiary teaching hospital in Sydney, Australia. Data were collected via semi-structured interviews and themes developed through inductive analysis. Three core themes emerged from the data: allied health professionals' level of "understanding of multidisciplinary roles", "facilitators and barriers to the dietitians role" such as patient complexity and training/education, and how these affected dietitians" "approaches to care". Although dietitians were recognised as the experts in nutrition, uncertainty was expressed about how to approach the nutritional management of this population due to the identified patient complexities. Further research exploring the patient experiences with dietitians is also warranted to provide defensible recommendations for the dietitian's role in the MDT management for these patients.

\subsection{Impact of a 4-Year State-Wide Salt Reduction Intervention on Salt Intake among Victorian Primary Schoolchildren}

\author{
Carley Grimes ${ }^{1}$, Kristy Bolton ${ }^{1}$, Kathy Trieu ${ }^{2}$, Jenny Reimers ${ }^{3}$, Sian Armstrong ${ }^{4}$, \\ Bruce Bolam ${ }^{5}$, Kelsey Beckford ${ }^{1}$, Elizabeth Dunford 2,6, Joseph Alvin Santos ${ }^{2}$, \\ Stephan Jan ${ }^{2}$, Jacqui Webster ${ }^{2}$, Bruce Neal ${ }^{2,7}$, Caryl Nowson ${ }^{1}$ and \\ Mark Woodward 2,7 \\ 1 Institute for Physical Activity and Nutrition, School of Exercise and Nutrition Sciences, \\ Geelong, Australia \\ 2 The George Institute for Global Health, University of New South Wales, \\ Newtown, Australia \\ 3 Victorian Health Promotion Foundation (VicHealth), Melbourne, Australia \\ 4 Heart Foundation, Melbourne, Australia \\ 5 Department of Health and Human Services, Melbourne, Australia \\ 6 Department of Nutrition, Gillings Global School of Public Health, The University of \\ North Carolina, Chapel Hill, USA \\ 7 The George Institute for Global Health, Imperial College of London, London, UK
}

In 2014, VicHealth formed the Victorian Salt Reduction Partnership to implement a 4-year multifaceted salt reduction intervention in Victoria. This study examined intervention effectiveness in reducing primary school-aged children's salt intake and discretionary salt use behaviours. Measures were taken pre (2010-13) and post (2018-19) intervention. Salt intake was measured via 24-h urinary sodium excretion, and discretionary salt behaviours were self-reported by the primary carer. Data were analysed with regression models adjusted for age, sex, socioeconomic disadvantage, and BMI z-score. Complete 24-h urine samples were collected from 827 children (baseline $n=666 ; 9.3 \pm$ (SEM) 0.1 y; $45 \%$ 
female vs. follow-up $n=161 ; 9.9 \pm 0.4 \mathrm{y} ; 48 \%$ female). Salt intake remained unchanged from baseline $(6.0 \pm 0.1 \mathrm{~g} / \mathrm{d})$ to follow-up $(6.1 \pm 0.4 \mathrm{~g} / \mathrm{d})(p=0.36)$. At both timepoints, approximately $70 \%$ of children exceeded sodium intake recommendations $(p=0.63)$. At follow-up, more parents $(14 \%, p=0.001)$ reported adding salt during cooking; child's use of table salt and placement of a salt shaker on the table remained unchanged. In conclusion, there were no improvements in children's salt consumption following a state-wide multifaceted intervention. Acknowledging the low sample size at follow-up, these findings suggest more intensive, sustained, and co-ordinated efforts between state and federal stakeholders, including government and food manufacturers/retailers, are required to lower children's salt intake.

\subsection{Outcomes of a Four-Year State-Wide Salt Reduction Initiative in Adults Living in Victoria, Australia}

Kristy A. Bolton ${ }^{1}$, Mark Woodward ${ }^{2,3}$, Caryl Nowson ${ }^{1}$, Bruce Bolam ${ }^{4}$, Joseph A. Santos ${ }^{2}$, Emalie Rosewarne ${ }^{2}$, Kathy Trieu ${ }^{2}$, Elizabeth Dunford 2,5, Stephen Jan ${ }^{2}$, Jacqui Webster ${ }^{2}$, Bruce Neal ${ }^{2,3}$, Jenny Reimers ${ }^{6}$, Sian Armstrong ${ }^{7}$ and Carley Grimes ${ }^{1}$

1 Institute for Physical Activity and Nutrition, School of Exercise and Nutrition Sciences, Deakin University, Geelong, Australia

2 The George Institute for Global Health, University of New South Wales, Newtown, Australia

3 The George Institute for Global Health, Imperial College London, London, UK

4 Department of Health and Human Services, Melbourne, Australia

5 Department of Nutrition, Gillings Global School of Public Health, The University of North Carolina, Chapel Hill, USA

6 Victorian Health Promotion Foundation (VicHealth), Carlton, Australia

7 Heart Foundation, Melbourne, Australia

The Victorian Salt Reduction Partnership aimed to reduce average salt intake of adults by $1 \mathrm{~g} /$ day over four years. This study measured change in salt intake (by 24 -h urinary excretion), sources of sodium intake (level of processing, purchase origin, by 24-h dietary recall) and self-report discretionary salt use behaviours pre- and post-intervention. Urinary data were collected pre- $(2016 / 17 ; n=339$, mean (SD) 47.6 (13.4) years, $54 \%$ female) and post-intervention (2020; $n=211,46$ (13.9) years, $50 \%$ female). A subsample completed a $24-\mathrm{h}$ dietary recall (pre- $n=144$, post-intervention $n=90$ ). There was no change in salt intake mean $(95 \%$ CI): $7.8(7.4,8.3) \mathrm{g} / \mathrm{d}$ pre- vs. $7.7(7.2,8.1) \mathrm{g} / \mathrm{d}$ post-intervention) (adjusted for age, sex, BMI, disadvantage, day of data collection). There were no differences in discretionary salt use behaviours. At post-intervention: discretionary foods contributed $32 \%$ of daily sodium; processed and ultraprocessed foods contributed $35 \%$ and $47 \%$ of daily sodium, respectively; $74 \%$ of sodium consumed originated from retail (supermarket) settings.

No change in salt intake or discretionary salt use behaviours were observed following a four-year state-wide intervention. This Victorian adult population still exceeds salt intake recommendations. A large proportion of salt intake derives from processed/ultraprocessed foods. Intensive efforts with strong government leadership and involvement from public health and food industry groups is required to reduce population salt intake.

2.39. Effects of Nutrition Interventions on Cardiovascular Disease Health Outcomes in Aboriginal and Torres Strait Islander Australians: A Scoping Review

\footnotetext{
Cassandra Brooks ${ }^{1}$, Hannah Melville ${ }^{1}$, Alyse Davies ${ }^{1}$, Bobby Porykali ${ }^{1,2,3}$, Julieann Coombes ${ }^{2,4}$ and Margaret Allman-Farinelli ${ }^{1}$

The Faculty of Medicine and Health, The University of Sydney, Sydney, Australia

2 Aboriginal \& Torres Strait Islander Health Program, The George Institute for Global

Health, Sydney, Australia

3 University of Technology, Sydney, Australia

4 The University of New South Wales, Sydney, Australia
} 
Since colonisation, Aboriginal and Torres Strait Islander peoples have forcefully been transitioned from a nutritious traditional diet to a Western diet, increasing the risk of developing cardiovascular disease (CVD). This review identifies and examines nutritional interventions aiming to improve CVD outcomes in Aboriginal and Torres Strait Islander peoples. Additionally, it appraises the interventions using an Aboriginal and Torres Strait Islander Quality Appraisal Tool. Searches were conducted across five databases and grey literature, applying no time limit. The results were screened by two researchers for eligibility, and data were extracted according to the Joanna Briggs Institute framework. All nutrition programs were examined across three outcome domains: anthropometric measures, biochemical/haematological biomarkers and other. Of the 21 programs included, 9 programs' evaluations were peer-reviewed, 5 had evaluations in grey literature, and 7 were not evaluated. Improved CVD health outcomes included decreased BMI, waist circumference, weight, blood pressure, and improved lipid profiles. Of the nine published programs critically appraised, four were identified as more culturally appropriate. Although more culturally appropriate programs did not show improved outcomes, an association between cultural appropriateness and program sustainability was identified. Future research should focus on sustainability within community through effective co-design with Aboriginal and Torres Strait Islander peoples.

2.40. Evaluation of Short and Long-Term Outcomes of OzHarvest's Nutrition Education and Skills Training (NEST) Programme in Vulnerable Adults

\section{Nell Vairinhos ${ }^{1}$, Elisha West ${ }^{2}$ and Karen Charlton ${ }^{1}$ \\ 1 University of Wollongong, Wollongong, Australia \\ 2 OzHarvest, Port Melbourne, Australia}

Food insecurity in Australia remains a significant public health problem, with estimates of around $24 \%$ of households being food insecure using a comprehensive 18 -item measurement tool. The OzHarvest NEST program delivers food literacy programs to vulnerable populations. A pre-post evaluation of the NEST program assessed changes in food security scores, food literacy (knowledge, confidence, behaviours), consumption of core food groups and discretionary foods/beverages of participants. Barriers and facilitators to sustained changes were investigated 6 months after program completion. A mixed methods approach evaluated pre-post surveys $(n=83)$ and 6-month follow-up in-depth interviews with program participants $(n=25)$. The prevalence of food insecurity reduced by $12 \%$, from $54 \%$ at baseline. Participants demonstrated improvements in food security status $(p=0.013)$, cooking confidence $(p=0.001)$, food preparation behaviours $(p=0.001)$, nutrition knowledge $(p=0.001)$ and vegetable $(p=0.001)$ and fruit consumption $(p=0.005)$. Six-month interviews identified that cost and access to food were the main barriers to sustain behaviour change, while enablers included donations from food charities. OzHarvest's NEST program results in improved food security and dietary behaviours, but sustained behaviour change is limited by underlying socioeconomic disadvantage.

\subsection{The New Normal for Food Insecurity? A Longitudinal Analysis over 1 Year during the} COVID-19 Pandemic in Tasmania, Australia

\section{Katherine Kent ${ }^{1,2}$, Sandra Murray ${ }^{2}$, Beth Penrose ${ }^{3}$, Stuart Auckland ${ }^{4}$, Elizabeth Lester ${ }^{5}$ and Denis Visentin ${ }^{2}$ \\ 1 School of Health Sciences, Western Sydney University, Campbelltown, Australia \\ 2 School of Health Sciences, University of Tasmania, Launceston, Australia \\ 3 Tasmanian Institute of Agriculture, University of Tasmania, Hobart, Australia \\ 4 Centre for Rural Health, University of Tasmania, Launceston, Australia \\ 5 Institute for Social Change, University of Tasmania, Hobart, Australia}

Food insecurity during the COVID-19 pandemic has been exacerbated by necessary public health restrictions. Tasmania has recorded no community transmission of COVID-19 
since May 2020. This study aimed to determine the change in prevalence and sociodemographic predictors of food insecurity throughout the COVID-19 pandemic in Tasmania. In May 2020 (T1; during lockdown), September 2020 (T2; easing restrictions) and May 2021 (T3; 1 year post-lockdown), cross-sectional, online surveys determined food insecurity in Tasmanian adults using the USDA Six-item Short Form, in addition to key sociodemographic questions. Multivariate binary logistic regression determined at-risk groups and evaluated changes in prevalence over time. The prevalence of food insecurity was $26.1 \%$ at $\mathrm{T} 1(n=1168), 18.0 \%$ at T2 $(n=1097)$ and $17.8 \%$ at T3 $(n=1100)$. Young adults, individuals with disabilities, families with dependents, Aboriginal and/or Torres Strait Islander people and temporary residents were at the highest risk across all timepoints. The prevalence of food insecurity reduced by $35 \%$ between T1 and T2 (AOR: $0.647 ; 95 \%$ CI: $0.514,0.814$; $p<0.001$ ) but was not significantly different between T2 and T3 (AOR: 1.069; 95\% CI: 0.834 , $1.371 ; p=0.598$ ). Our results indicate that despite easing social distancing restrictions, the prevalence of food insecurity did not recover to pre-pandemic levels 12 months following a lockdown.

\subsection{Australian Parents' Involvement and Enjoyment in Food-Related Interaction with Their} Children during the COVID-19 Pandemic

\section{Janandani Nanayakkara ${ }^{1}$, Gail Boddy ${ }^{1}$, Gozde Aydin ${ }^{1}$, Krupa Kombanda ${ }^{1}$, Christel Larsson ${ }^{2}$, Anthony Worsley ${ }^{3}$, Claire Margerison ${ }^{1}$ and Alison Booth ${ }^{1}$ \\ 1 Institute for Physical Activity and Nutrition, School of Exercise and Nutrition Sciences, Deakin University, Geelong, Australia \\ 2 Department of Food and Nutrition, and Sport Science, University of Gothenburg, Läroverksgatan 5, Box 300, 405 30, Göteborg, Sweden \\ 3 School of Exercise and Nutrition Sciences, Deakin University, Geelong, Australia}

The COVID-19-pandemic-associated lockdowns/restrictions resulted in many children transitioning to remote learning (RL) and parents working from home. This study explored parents' involvement and enjoyment in food-related interactions with their children during COVID-19-associated lockdowns/restrictions and their perceptions of changes in children's lunch during RL. An online survey was conducted in 2020. Parents' $(n=136)$ quantitative responses and written comments were analysed using descriptive statistics and thematic analysis, respectively. Most parents (62\%) reported that they interacted more with their children about food during the pandemic compared to pre-pandemic times. These interactions included cooking, menu planning, eating, conversations around food, and gardening. Most parents (74\%) prepared meals with their children during the pandemic, and most of them $(89 \%)$ reported that they enjoyed it. Most parents $(n=91$ out of 121$)$ perceived that their children's lunches during RL were different to when attending school in person. These differences included: eating more hot lunches, improved quality, and no set lunchtime. Overall, during the pandemic-related lockdowns/restrictions, parents experienced more positive food-related interactions with their children than before the pandemic, and there were changes in the quality of food eaten by children during periods of RL.

\subsection{No Effect of TAS2R38 Bitter Taste Status on Vegetable Acceptance in a Child/Adult Cohort}

Astrid A.M. Poelman, Jess Heffernan, Damian Frank, Uday Piysiri and Nicholas Archer

CSIRO, North Ryde, Australia

A commonly reported marker used by nutrigenomic companies is the bitter taste response of the TAS2R38 receptor for dietary advice on vegetable intake. Further understanding of the effect of TAS2R38 bitter taste on vegetable acceptance is required and whether age-related differences exist. This study investigated vegetable acceptance in a child/adult cohort as factor of TAS2R38 taster status. We hypothesized where mediating genetic factors reduced vegetable acceptance, this effect would be moderated by age, with 
a stronger effect in children. A cohort of 98 child/parent dyads were genotyped for the common variants of TAS2R28 and split into bitter taster and non-taster groups. Acceptance for 33 vegetables (with a range of sensory properties) was measured using 9-point hedonic scales. Vegetable acceptance was lower in children than adults. Overall, TAS2R38 status did not affect the acceptance of vegetables with higher bitterness. Differences in acceptance based on TAS2R38 status were observed for three non-bitter vegetables in children (two liked more by bitter tasters and one less) and one slightly bitter vegetable in adults (liked more by bitter tasters). In conclusion, this study shows no evidence of reduced acceptance of vegetables by bitter tasters, limiting the ability to use this marker for advice on vegetable intake.

2.44. Examining the Effect of a Meal Timing Intervention in Shift Workers on Post Meal PBMC Transcriptomic Responses

\author{
Rochelle Davis ${ }^{1}$, Maxine P. Bonham ${ }^{1}$, Gloria K.W. Leung ${ }^{1}$, Chiara Murgia 1,2, \\ Morag J. Young ${ }^{3}$ and Catherine E. Huggins ${ }^{1}$ \\ 1 Department of Nutrition, Dietetics, and Food, Monash University, \\ Notting Hill, Australia \\ 2 School of Agriculture and Food, The University of Melbourne, Melbourne, Australia \\ 3 Baker Heart and Diabetes Institute, Melbourne, Australia
}

Night shift workers face greater cardiometabolic disease risk, facilitated by circadian disruption caused by frequent night eating. The aim of this study was to explore the effect of food avoidance overnight in shift workers with abdominal obesity on the blood transcriptome. In a randomised cross-over trial, 19 shift workers completed 4 weeks of intervention (fasting between 1 a.m. and 6 a.m.) and control (ad libitum intake) conditions with a 2-week wash-out. At the end of each condition, peripheral blood mononuclear cells were isolated from samples collected at fasting and 2 and $4 \mathrm{~h}$ after the consumption of a high-fat metabolic challenge meal. Total RNA was extracted (QIAGEN GmbH) and sequenced (Illumina HiSeq). Differential gene expression was analysed (DESeq2, V1.6.3) with functional analysis explored in PathVisio (V3.3.0). After the intervention versus control condition, there were no differences in fasting gene expression, with 372 genes differentially expressed postprandially. These genes were associated with 80 biological pathways that were enriched, $60 \%$ of the top 10 involved in mitochondrial homeostasis and energy metabolism. The implementation of a short overnight fast in shift workers resulted in the activation of genes involved in adaptation to cellular energy demands and could be a feasible dietary strategy to improve their metabolic health.

\title{
2.45. Exploring the Use of Screens during Family Mealtimes in Australian Families of Low Socioeconomic Position with Young Children
}

\section{Eloise-Kate Litterbach, Rachel Laws, Karen Campbell, Miaobing Zheng and Alison Spence}

Institute for Physical Activity and Nutrition, School of Exercise and Nutrition Sciences, Deakin University, Geelong, Australia

Mealtime screen use (MSU) is common, particularly in families of low socioeconomic position (SEP), and can negate some of the nutritional and social benefits associated with family meals. Qualitative interviews with mothers of low SEP were conducted via Zoom, utilising semi-structured interview schedules and Photo Interviewing techniques. Transcripts were analysed thematically using a constructivist paradigm. Although a strong theme among the 25 participants was an eagerness for reducing family MSU, mothers considered MSU to have both advantages and disadvantages. Perceived advantages included relaxing the mood of mealtimes, reducing food negotiations, and managing picky eating behaviours. Themes relating to the disadvantages of MSU included children's social disengagement and mindless eating, and for some, MSU increased fussy food behaviours. Some mothers acknowledged that their own phone use during mealtimes contributed to 
normalising MSU behaviours in their children. Themes suggest that MSU is likely linked with a range of child behaviours and parenting practices, including distraction, that have the potential to negatively influence dietary intake and social engagement. Parents' desire to reduce family MSU provides an important opportunity to determine how to best support parents to achieve this and to potentially improve child health outcomes.

\title{
2.46. Effects of Acute Feeding with Almonds Compared to a Carbohydrate-Based Snack on Appetite
}

\author{
Sharayah Carter ${ }^{1}$, Alison Hill ${ }^{1}$, Jonathan Buckley ${ }^{1}$, Sze-Yen Tan ${ }^{2}$, Geraint Rogers ${ }^{3}$ \\ and Alison Coates ${ }^{1}$ \\ 1 University of South Australia, Adelaide, Australia \\ 2 Institute for Physical Activity and Nutrition, School of Exercise and Nutrition Sciences, \\ Deakin University, Geelong, Australia \\ 3 Flinders University, Bedford Park, Australia
}

Despite being high in energy, nut consumption does not increase the risk of weight gain, which may be explained by appetite-regulating effects. This study compared postprandial changes in appetite-regulating hormones and subjective hunger/satiety ratings over $120 \mathrm{~min}$, followed by a food buffet after the consumption of an isocaloric snack (15\% energy) of almonds (AL, $n=67$ ) or a carbohydrate-rich snack bar (SB, $n=70)$. This is a sub-analysis of baseline assessments of a larger randomised controlled trial in overweight and obese (BMI $27.5-34.9 \mathrm{~kg} / \mathrm{m}^{2}$ ) adults (25-65 years). Postprandial C-peptide area under the curve (AUC) was $46 \%$ lower with AL compared to SB $(p<0.001)$. Glucose-dependent insulinotropic polypeptide, glucagon and pancreatic polypeptide AUC were higher with AL compared to SB $(17 \%, p=0.002 ; 39 \% p<0.001 ; 48 \% p<0.001$, respectively). Ghrelin, glucagon-like peptide-1, leptin, polypeptide $Y Y$ and cholecystokinin AUCs were not different between groups. Subjective hunger/satiety ratings and energy intake in a sub-set of participants ( $\mathrm{AL}, n=49$; $\mathrm{SB}, n=48$ ) did not differ between groups. More favourable gut hormonal responses of AL did not translate into reduced energy consumption acutely, and therefore, future studies should investigate the implications on longer-term appetite regulation and weight management.

\subsection{Associations between Dietary Lifestyle and Australian's Willingness to Eat Insects}

\section{Indee Hopkins, Jessica Danaher, Asgar Farahnaky, Harsharn Gill and Lisa Newman RMIT University, Bundoora, Australia}

Increasing global populations and the limitations on finite resources will place greater pressure on an already strained food system. Exacerbated by changing dietary preference, protein is of particular concern. Insect protein is nutritious and sustainable, yet its uptake by the Australian consumers has been slow. This study aimed to explore the dietary lifestyle choices of Australians on influence on willingness to eat insects. Participants $(n=601$; $76.2 \%$ female, $23.8 \%$ male; aged $18-35$ years $40.8 \%$, 36-54 years $43.6 \%$, $\geq 55$ years $15.6 \%$ ) completed an online survey about their dietary lifestyle choices, current protein intake and willingness to eat insects. Protein sourced from dairy (milk, yoghurt) was the most frequently consumed, with $58.2 \%$ of participants reporting daily consumption. A strong association was observed between participants' dietary lifestyle and their willingness to eat insects, with flexitarians $(36.7 \%)$ being more likely to eat insects than any other dietary preference $(p<0.001)$. The outcomes of this research will provide a greater understanding of the influence of dietary lifestyle choices on willingness to eat insect and direct tailored strategies to improve perceptions towards insects as food and facilitate a greater acceptance of the alternative protein source. 


\title{
2.48. Applying the Living and Eating for Health Segments (LEHS) in a Group of Victorian
} Young Adults

\author{
Sarah Eddy ${ }^{1}$, Megan S.C. Lim ${ }^{1}$, Annika Molenaar ${ }^{2}$, Eva Jenkins ${ }^{2}$ and \\ Tracy A. McCaffrey ${ }^{2}$ \\ 1 Burnet Institute, Melbourne, Australia \\ 2 Monash University, Notting Hill, Australia
}

We developed Living and Eating for Health Segments (LEHS) based on attitudes and values towards healthy eating and showed demographic differences between groups. This study aimed to determine whether LEHS profiles exist in an online survey of 15-29-year-olds in Victoria $(n=1001)$. Participants selected one LEHS, Lifestyle Mavens (LM), Health Conscious (HC), Aspirational Healthy Eaters (AHE), Balanced All-Rounders (BAR), Contemplating Another Day (CAD), Blissfully Unconcerned (BU), and answered demographic questions. In 2021, 84\% of participants were born in Australia, 59\% identified as female and $6 \%$ as non-binary, with $70 \%$ currently studying at high school, university or TAFE. The samples were segmented with 38\% BAR, 24\% AHE, 16\% CAD, 16\% HC, 3\% BU, and 3\% LM. The BAR were 65\% female; AHE, CAD, and HC included slightly more females (54\%). BU included slightly more males (46\%); and LM were 69\% males. LM (39\%) and HC (36\%) were more likely to live with friends or housemates $(p<0.001)$, whereas BU $(68 \%)$, CAD (56\%), AHE (53\%), and BAR (53\%) were more likely to live with their parents $(p=0.002)$. Preliminary results show that LEHS are applicable in other groups and could help to understand participant's attitudes and values towards health within future interventions.

\section{Poster Presentations}

3.1. Parental Perceptions of Their Children's Diet and Academic Performance in the First Three Years of School: A Mixed-Method Analysis

\section{Jameela Ho}

Western Sydney University, Campbelltown, Australia

Good nutrition for optimal health and educational outcomes would provide opportunity for children to not only survive but thrive in today's society. However, despite the Australian Government providing healthy eating guidelines, the quality of Australian children's diet often remains poor, low in fruit and vegetables but high in fats and sugars. This study utilised a qualitative questionnaire on parents' perspectives of their child's diet and academic performance. It also utilised the quantitative Australian Child and Adolescent Eating Survey. The results found that even though parents' value healthy eating and want their children to eat healthy, their child's diet quality is poor and did not meet Australian Dietary Guidelines. Notably for participating parents from culturally diverse backgrounds, there was a disconnect between their wanting children to eat healthy and children's actual diet quality. Culturally diverse parents also demonstrated limited knowledge by not describing a connection between children's diet and academic performance or a clear understanding of what a healthy diet should consist of for school-age children. There is a need for intervention, especially in schools with culturally diverse backgrounds, to ensure parents are aware of how diet can contribute to children's academic performance and what a 'healthy school diet' includes.

3.2. Nutritional Adequacy and Patient Perceptions of the Inpatient Haemodialysis Menu: A Mixed Methods Case Series

Fatima F.A. Al Nakeeb ${ }^{1}$, Kelly K.L. Lambert ${ }^{1}$ and Kathleen K.N. Neal ${ }^{2}$

1 Nutrition and Dietetics, University of Wollongong, Wollongong, Australia

2 Nutrition and Dietetics, Liverpool Hospital, NSW Health, Sydney, Australia

Patients receiving haemodialysis are at increased risk of malnutrition due to their increased nutritional needs and complex nutrition prescription. Malnutrition is common in the hospital setting; however, research that quantifies the dietary intake and explores 
the perceptions of haemodialysis inpatients regarding intake is limited. Eight participants were purposively sampled on the renal ward at Liverpool Hospital between April and June 2021. Semi-structured interviews were conducted to quantify the dietary intake of participants and their perceptions on the adequacy and suitability of the inpatient haemodialysis menu. The electronic menu system and Foodworks ${ }^{\mathrm{TM}}$ were used to quantify dietary intake and simple thematic analysis used to identify dominant themes. Inadequate energy and protein intake was present in half of the study sample. Passive acceptance of the menu, environmental and individual barriers, and culture contributed to missed food opportunities. This, in turn, impacted the patient experience and resulted in inadequate intake. The standard inpatient haemodialysis menu did not provide adequate energy, sodium, potassium, vitamin $\mathrm{C}$ and fibre. The nutritional profile of the haemodialysis menu impacts dietary intake and the experiences of haemodialysis inpatients. A review of the inpatient haemodialysis diet to optimise dietary quality of the menu and further research about patient perceptions is recommended.

\title{
3.3. Interventions to Improve Dietary Intakes in Indigenous Children: A Systematic Review
}

\section{Oyepeju Onifade ${ }^{1}$, Lucy Kocanda ${ }^{1}$, Tracy Schumacher ${ }^{2}$, Megan Rollo ${ }^{3}$, Kym Rae $^{4}$ and Kirsty Pringle ${ }^{5}$ \\ 1 School of Medicine and Public Health, University of Newcastle, Callaghan, Australia \\ 2 Department of Rural Health, School of Medicine and Public Health, Faculty of Health and Medicine, University of Newcastle, Tamworth, Australia \\ 3 School of Health Sciences, University of Newcastle, Callaghan, Australia \\ 4 Mater Research Institute, South Brisbane, Australia \\ 5 School of Biomedicine and Pharmacy, University of Newcastle, Callaghan, Australia}

Nutrition is an important component of the first 1000 days of life, particularly among Indigenous populations. The aim of this review was to systematically assess the effectiveness of interventions designed to optimise dietary intake and/or nutrition-related behaviours among Indigenous infants globally and to identify whether Indigenous populations were involved in the co-design of the intervention. A database search was conducted across six databases and data on study aims and design, target population, geographical location, the health condition of the participants, intervention characteristics, co-design, and outcomes were extracted. A narrative synthesis of acceptability and effects of the interventions and participants' involvement in the study design were highlighted. Population-based intervention studies were included in this review. Of the 2784 studies identified, 3 studies that were conducted among two Indigenous tribes in Guatemala and the United States of America met the inclusion criteria. Two studies reported the food and nutrient intake of participants, with one study showing an improvement in the dietary intake of the infants. Only one study reported community participation in the study design, intervention design and implementation, and acceptability of the intervention by the participants. Engaging Indigenous communities throughout the entire process of nutrition interventions could have beneficial effects through improved outcomes.

\subsection{A Comparison of Diet Quality in a Sample of Rural and Urban Australian Adults}

\author{
Rebekah Pullen ${ }^{1}$, Katherine Kent ${ }^{2}$, Matt Sharman ${ }^{1}$, Tracy Schumacher ${ }^{3}$ and \\ Leanne Brown ${ }^{3}$ \\ 1 School of Health Sciences, University of Tasmania, Launceston, Australia \\ 2 School of Health Sciences, Western Sydney University, Campbelltown, Australia \\ 3 Department of Rural Health, The University of Newcastle, Tamworth, Australia
}

Diet quality in rural Australia is under-researched. Characterising disparities in diet quality between rural and urban populations may inform targeted interventions in at-risk groups. A cross-sectional study aimed to determine the relationship between diet quality, rurality and sociodemographic characteristics in a sample of Australian adults. Participants were recruited at rural gala events. Diet quality was measured using the Healthy Eating 
Quiz to generate the Australian Recommended Foods Score (ARFS). ARFS was compared by rurality and sociodemographic characteristics using multivariate regression. Participants ( $n=247 ; 53 \%$ female) had a mean ARFS of $34.5 \pm 9.0$. There was no significant effect of rurality on ARFS ( $\beta$-coefficient $=-1.4 ; 95 \% \mathrm{CI}-3.3,-0.5)$. Compared to participants aged 18-30 years, higher ARFS was evident for those aged 31-50 ( $\beta=5.3 ; 95 \%$ CI $0.2,10.3), 51-70$ ( $\beta=4.2 ; 95 \%$ CI 1.0, 8.3) and $>71$ years $(\beta=5.3 ; 95 \%$ CI 1.4, 11.3). Compared to those living alone, participants living with a partner $(\beta=5.7 ; 95 \%$ CI $2.5,8.9)$ and families with children $(\beta=6.1 ; 95 \%$ CI $1.9,10.3)$ had higher ARFS. ARFS was significantly lower with each additional self-reported chronic health condition $(\beta=-1.8 ; 95 \%$ CI $-3.0,-0.5)$. Our results indicate that diet quality was low and age, living arrangements and chronic health conditions, but not rurality, influenced diet quality in a sample of Australian adults.

\subsection{Consumer Experiences of Food Environments from 119 Countries during COVID-19}

Lydia O'Meara ${ }^{1}$, Christopher Turner ${ }^{1}$, Denise Costa Coitinho ${ }^{2}$ and Stineke Oenema ${ }^{2}$

1 Natural Resources Institute, University of Greenwich, London, UK

2 United Nations Nutrition, Rome, Italy

This study investigates consumer experiences of food environments and food acquisition practices during the COVID-19 pandemic, drawing from a rapid online survey of 2015 individuals from 119 countries in April 2020. The findings reveal common global experiences related to food availability and accessibility, food prices and affordability, food acquisition practices, and food preparation and consumption. The importance of community food participation, food sharing, and resource allocation are highlighted, along with increasing awareness of healthy diets and food waste. We identify 10 synergistic policy entry points to: (1) build resilient and equitable food environments resistant to stresses and shocks; (2) harness positive dietary-related behaviours manifested during the pandemic; and (3) mitigate the projected nutrition crisis and promote sustainable healthy diets for all.

3.6. The Effects of the COVID-19 Pandemic on Food Security in Australia and Implications for Program Planning: A Scoping Review

\section{Serena Louie, Yumeng Shi and Margaret Allman-Farinelli}

University of Sydney, Newtown, Australia

The COVID-19 pandemic has disrupted the Australian food supply, with potential ramifications for food security. This scoping review aimed to synthesise current evidence on the prevalence of food insecurity and changes to factors related to food insecurity during the COVID-19 pandemic in Australia. A systematic search of eight electronic databases was conducted. Included studies were written in English, published in 2020 and later and examined food security status in Australia during the COVID-19 pandemic and/or factors associated with food insecurity in free-living Australian residents. In total, 689 articles and 11 from reference lists were identified and screened, with 9 studies being included. All studies indicated that the prevalence of food insecurity had increased due to adverse changes to food availability, accessibility, and affordability caused by the pandemic. There has been a shift in the demographic profile of the food-insecure population (e.g., international students and newly unemployed casual workers) due to a downturn in economic and employment circumstances during the pandemic. COVID-19 has exacerbated vulnerabilities in the Australian food supply and food security. Suggested future actions include coordinated national and community responses that improve the resilience of the local food supply and address social, economic and geographic determinants of food insecurity. 
3.7. Big Data in Public Health: Using Sentiment Analysis to Understand Food Waste Discussions on Twitter

\author{
Eva L. Jenkins ${ }^{1}$, Dickson Lukose ${ }^{2}$, Linda Brennan ${ }^{3}$, Annika Molenaar ${ }^{1}$ and \\ Tracy A. McCaffrey ${ }^{1}$ \\ 1 Department of Nutrition, Dietetics and Food, Monash University, \\ Notting Hill, Australia \\ 2 Monash Data Futures Institute, Monash University, Clayton, Australia \\ 3 School of Media and Communication, RMIT University, Melbourne, Australia
}

Food waste is a growing global challenge, with one-third of food produced being lost or wasted, resulting in detrimental impacts on the environment and economy. Using social media data to explore food waste discussions could provide a deeper understanding of the current conversation and inform future communication efforts. However, computer science techniques are yet to be explored in this domain. This study explored the sentiment of the food waste discussion on Twitter. Australian tweets containing keywords related to food waste were collected from the Twitter API from 2019-2020. Sentiment was analysed using the Valence Aware Dictionary and Sentiment Reasoner (VADER) sentiment engine. A total of 37,390 tweets were collected, with $54.3 \%$ from 2019 and $45.7 \%$ from 2020 . The most prevalent sentiment was negative $(41.7 \%)$, followed by neutral $(28.9 \%)$, positive $(27.2 \%)$, very negative $(1.1 \%)$, and very positive $(1.0 \%)$. Overall, the discussion of food waste on Twitter throughout 2019 and 2020 was more commonly negative than neutral or positive. Future analysis should consider looking at the predominant emotions expressed in the tweets to understand more about the nuances in food waste discussions on Twitter.

\title{
3.8. The Development of the DIGIFOOD Dashboard to Monitor the Impact of Online Food Delivery Services on the Food Environment
}

\section{Sisi Jia ${ }^{1}$, Stephanie Partridge ${ }^{1}$ and Alice Gibson ${ }^{2}$}

1 School of Health Sciences, University of Sydney, Sydney, Australia

2 Menzies Centre for Health Policy and Economics, University of Sydney,

Sydney, Australia

Online food delivery services (OFDS) are potentially exacerbating takeaway food accessibility and consumption. To monitor the impact of OFDS on the food environment in Australia, we developed the DIGIFOOD dashboard. In January 2021, primary data for the DIGIFOOD dashboard were acquired from the market-leading OFDS in Australia via web-scraping services. Data variables included food outlets, geographical location and outlet category descriptors. The Food Environment Score was applied to determine the healthiness of available outlets. A data-driven dashboard was produced to map and colour-code the healthiness of online food outlets across Australia using a traffic-light system. To date, 222,425 food outlets have been mapped on the DIGIFOOD Dashboard. The search functions of the dashboard enable users to interact and observe the healthiness scores of online food outlets in any state, region, postcode or locality. The dashboard also provides a breakdown of the number of food outlets accessible online via their search postcode versus the number of locally accessible food outlets in the physical postcode. The DIGIFOOD Dashboard provides a lay summary of the healthiness of current digital food environment driven by the proliferation of OFDS. Further research will be conducted to analyse differences in healthiness across geographical areas and over time.

\subsection{Why Do Young Australians Eat Seaweed? Factors Influencing the Consumption of Seaweed in} Young Australian Adults

Mikaela Young ${ }^{1}$, Nicholas Paul ${ }^{2}$, Dawn Birch ${ }^{3}$ and Libby Swanepoel ${ }^{1}$

1 School of Behavioural and Health Science, University of the Sunshine Coast, Maroochydore, Australia

2 School of Science, Technology and Engineering, University of the Sunshine Coast, Maroochydore, Australia 
3 School of Business and Creative Industries, University of the Sunshine Coast, Maroochydore, Australia

Seaweed has been traditionally consumed in Asian and Pacific cultures; however, it is not commonly eaten in Western societies. Edible seaweeds provide a range of nutritional benefits to the diet and are advantageous over terrestrial crops when comparing the environmental demand and sustainability of cultivation. The study's aim is to explore the motivations that drive young Australians to eat seaweed. This is necessary to better advise seaweed aquaculture development, wild-harvest practices, industry food product development and consumer marketing strategies. An observational cross-sectional online survey with $n=1000$ young (19-30 years) Australian seaweed consumers was conducted. The 21-item survey included a range of closed-ended, open-ended and Likert-scale responses. The majority of respondents were female (88.6\%), had completed tertiary-level education or above $(55.8 \%)$, and lived in a metropolitan area $(77.8 \%)$. The most known varieties of seaweed included nori $(35.8 \%)$, wakame $(18.7 \%)$, and kelp $(12.5 \%)$, with nori $(28.4 \%)$, wakame $(12.3 \%)$, and roasted seaweed snacks (11.1\%) being the most consumed. Common themes emerged for drivers for consumption, including health, environmental, social, packaging, and convenience factors. Exploration of the influences on food-related decisions that contribute to current seaweed consumption provides greater understanding to guide the promotion of seaweed to existing and future consumers.

\subsection{A Cross-Sectional Audit of Sorghum in Breakfast Cereals and Snack Bars in Australian Supermarkets}

\section{Anita Stefoska-Needham ${ }^{1}$ and Cecily Ducksbury ${ }^{2}$ \\ 1 University of Wollongong, North Wollongong, Australia \\ 2 Nutrition, Kellogg Australia, Sydney, Australia}

Sorghum grains may play a role in mechanisms that protect against the development of chronic diseases. However, sorghum is not widely consumed by Australians, and its presence in common food products is unknown. We conducted a study to examine the utilisation of sorghum in common food products, notably, breakfast cereals and snack bars, in a cross-section of five supermarkets over a seven-day period in February 2020 in the Illawarra. Details relating to ingredients, food format, brand and product name were recorded. Sorghum was present in $6.1 \%(23 / 379)$ of breakfast cereals in a variety of formats such as extruded shapes, flour and puffed grains. In $8.7 \%$ of these, sorghum was listed as the first ingredient (greatest contribution by weight). Sorghum was utilised in $2 \%$ (6 of 298) of snack bars, mainly as puffed sorghum, and was listed in the fourth or subsequent position in the ingredient lists for all. "Sorghum" did not appear in the name of any audited products. In conclusion, sorghum is present in a very small number of breakfast cereals and snack bars in Australian supermarkets. To encourage consumer adoption and the recognition of sorghum, and to expand the product range, more investment in sorghum food innovation and marketing is needed.

3.11. A Cross-Sectional Study of Diet Diversity in a Sample of Children Aged 0-5 Years from Culturally and Linguistically Diverse Backgrounds in Western Sydney

\section{Anjana Iyer ${ }^{1}$, Katherine Kent ${ }^{2}$ and Catharine Fleming ${ }^{2}$}

1 School of Science, Western Sydney University, Penrith, Australia

2 School of Health Sciences, Western Sydney University, Campbelltown, Australia

Childhood obesity remains a major public health concern, especially for children from culturally and linguistically diverse (CALD) backgrounds. Yet, research on dietary patterns in children from CALD backgrounds in Western Sydney is especially sparse. This cross-sectional study aimed to characterise breastfeeding history, nutrient intake and diet diversity in a sample of children aged 0-5 years from CALD backgrounds in Western Sydney. Nutrient intake and diet diversity were determined using $24 \mathrm{~h}$ recalls, 
and a survey collected breastfeeding history. Nutrient adequacy was determined using Australian Nutrient Reference Values, and diet diversity scores (DDS) were calculated using the WHO's Minimum Diet Diversity measure. Dietary data were analysed using descriptive statistics, Chi-square statistics and one-way ANOVA. Children $(n=29$; median age 26 (6-66) months) had a median DDS of $5(3-7)$. Most children $(93.1 \%)$ received breastmilk as their first feed, and $47.8 \%$ continued breastfeeding beyond 12 months. The recommended daily intakes for most nutrients were met by most children, except for iron (34.5\%). Most children (>75.0\%) exceeded their estimated energy requirements and recommended saturated fat intake, which was further exceeded by children breastfed beyond 12 months $(p=0.01)$. Our results indicate a need for targeted interventions to improve children's diets in Western Sydney.

3.12. Developing Texture Modified Foods for Aged Care with Improved Sensory Properties: From Concept Design to Consumer Testing

\author{
Janne Beelen ${ }^{1}$, Maeva Broch ${ }^{2}$, Ciara McDonnell ${ }^{3}$ and Aarti Tobin ${ }^{3}$ \\ 1 CSIRO Health and Biosecurity, North Ryde, Australia \\ 2 CSIRO Agriculture and Food, North Ryde, Australia \\ 3 CSIRO Agriculture and Food, Coopers Plains, Australia
}

As a result of an aging population, there will be an increase in age-related issues with eating, such as dysphagia, putting people at risk of undernutrition. Dysphagia sufferers require texture-modified foods (TMF), which can be subject to quality and flavour issues. Sensory issues were previously studied in the project, and this research aimed to optimise TMF's sensory properties for use in aged care. Two concepts were developed into a series of prototypes: 8 restructured steaks and 10 minced and moist peas with various textures that were profiled by a trained sensory panel. Steaks differed significantly in eight mouthfeel and oral breakdown properties, with salt content having the largest effect. Fifteen attributes differed significantly between pea prototypes, with the particle size affecting mouthfeel properties and heat treatment affecting brightness and pea flavour. The results were used to select prototypes for a consumer acceptance test (CAT) with aged care residents. CAT results provided further insights into product optimisation, which will be presented. This research was an initial study to optimise the sensory quality of TMF for aged care. Future studies will include optimising the nutritional profile of these products, ensuring dysphagia sufferers are offered the most nutritious and palatable foods.

\title{
3.13. The Feasibility of Providing a Cooked School Lunch Program in Tasmania
}

Kylie J. Smith ${ }^{1}$, Julie Dunabin ${ }^{2}$, Laura Cini ${ }^{2}$, Claire Galloway ${ }^{3}$, Natasha Meerding ${ }^{3}$, Kate DePaoli ${ }^{3}$ and Monique Reardon ${ }^{3}$

1 Menzies Institute for Medical Research, University of Tasmania, Hobart, Australia

2 Tasmanian School Canteen Association, Hobart, Australia

3 Public Health Services, Department of Health Tasmania, Hobart, Australia

This study examined the feasibility of providing a cooked school lunch program in Tasmania. In total, 3 schools received financial and practical support to provide healthy cooked lunches to 201 children for 20 days. The meals were consistent with the 2013 Australian Dietary Guidelines and cooked from scratch with more than half of the ingredients grown in Tasmania. Children had $20 \mathrm{~min}$ to sit and eat the meal together. Children, parents, and staff $(n=160)$ provided feedback through discussion groups, interviews, and surveys. Children liked the variety, trying new foods, and sitting with friends. Parents appreciated not preparing lunchboxes, that 'picky eaters' were trying new foods, and children were more settled after school. Two schools reported an increase in school attendance and calmer children. A few children disliked inconsistent serving sizes, only having one lunch option each day, and meals served late. Challenges included insufficient infrastructure (e.g., need commercial dishwashers, larger ovens, more crockery/cutlery) and adjusting teaching schedules to allow more time for eating lunch. Providing school lunches was feasible, 
valued, and seen as an equitable opportunity for all children to eat healthy food. A greater understanding of the benefits and challenges will be examined in a larger trial ( 30 schools) during 2022-23.

\subsection{Associations of Social Interaction and Food Preparation Context on Quality of Meals and Snacks Consumed by Young Adults}

\section{Virginia Chan ${ }^{1}$, Lyndal Wellard ${ }^{2}$, Alyse Davies ${ }^{1}$, Wendy Watson ${ }^{2}$, Clare Hughes ${ }^{2}$, Leanne Wang ${ }^{1}$ and Margaret Allman-Farinelli ${ }^{1}$ \\ 1 University of Sydney, Camperdown, Australia \\ 2 Cancer Prevention and Advocacy Division, Cancer Council NSW, \\ Woolloomooloo, Australia}

Food prepared outside the home and socializing may be important determinants of dietary consumption. The aim of this study was to examine the impact of these two factors on the quality of meals and snacks eaten by young adults using objective measurements. A total of 133 18-30-year-olds wore an Autographer camera for 3 days during waking hours. The wide-angled lens camera took an image every $30 \mathrm{~s}$, capturing a participantorientated view of daily experiences. Images identified 1832 eating occasions that were annotated for place of food preparation, i.e., home cooked or prepared outside home; whether the meal or snack was predominantly 5 food groups or discretionary foods; and social interactions with others. Logistic regression was used to examine associations of the contextual factors and meal quality stratified by meal type. Home-prepared meals and snacks were much more likely to be five food groups than discretionary (breakfast $\mathrm{OR}=2.5$ (95\% CI: 1.1-5.3), lunch OR = 4.4 (95\% CI: 2.6-7.4), dinner OR = 12.0 (95\% CI: 6.5-22.0), and snacks OR $=2.5$ (95\% CI: 1.8-3.6)). However, no associations with social interaction were detected. Home-cooked meals have a positive influence on food quality.

3.15. Dietary Patterns Trajectories and Nutritional Status from Preconception to Late-Pregnancy in a Singapore Preconception Cohort Study

Jun S. Lai ${ }^{1}$, Jason Loh ${ }^{1}$, Geeta Appannah ${ }^{2}$, Shan Xuan Lim ${ }^{3}$, Marjorelee T. Colega ${ }^{1}$, Keith M. Godfrey ${ }^{4}$, Fabian Yap 5,6, Yap Seng Chong ${ }^{1,7}$, Johan G. Eriksson 1,7,8, Jerry K.Y. Chan ${ }^{6,9}$, Shiao-yng Chan ${ }^{1,7}$ and Mary F.F. Chong ${ }^{1,3}$

1 Singapore Institute for Clinical Sciences, ASTAR, Singapore

2 Department of Nutrition, Faculty of Medicine and Health Sciences, Universiti Putra Malaysia, Serdang, Selangor, Malaysia.

3 Saw Swee Hock School of Public Health, National University of Singapore and National University Health System, Singapore

4 MRC Lifecourse Epidemiology Centre \& NIHR Southampton Biomedical Research Centre, 4MRC Lifecourse Epidemiolo, University of Southampton \& University Hospital Southampton NHS Foundation Trust, Southampton, UK

5 Department of Paediatric Endocrinology, KK Women's and Children's Hospital, Singapore

6 Duke-NUS Medical School, Singapore

7 Department of Obstetrics and Gynaecology, Yong Loo Lin School of Medicine, National University of Singapore, Singapore

8 Department of General Practice and Primary Health Care, University of Helsinki and Helsinki University Hospital, \& Folkhälsan Research Center, University of Helsinki, Helsinki, Finland

9 Department of Reproductive Medicine, KK Women's and Children's Hospital, Singapore

The extent to which women change their diet from preconception to pregnancy is unclear. In a Singapore preconception cohort (S-PRESTO), we examined dietary pattern trajectories and plasma micronutrients from preconception to late-pregnancy. Dietary intakes were assessed with food frequency questionnaires at preconception and 19-20 weeks' and 
34-36 weeks' gestation. We characterised dietary patterns at all follow-ups using factor analysis and derived trajectories for each pattern using group-based modelling. Plasma concentrations of vitamins A, D and B were quantified at preconception and 26-28 weeks' gestation. Among the 325 women included, we observed minimal changes in dietary patterns over time. For each of the 'Less-healthy' and 'Prudent-Singaporean' dietary patterns, two trajectories (consistently high vs. consistently average adherence) were identified. Compared to the consistently average trajectories, women in the consistently high 'Lesshealthy' trajectory $(n=37,11 \%)$ tended to have persistent vitamin D deficiency and lower vitamins B1 and B6 concentrations, whilst vitamin D deficiency was less likely and vitamin A concentrations were higher among women in the consistently-high 'Prudent-Singaporean' trajectory $(n=96,30 \%)$. Our study identified a group of women with a consistently 'Lesshealthy' diet (higher intakes of fast food, fried snacks and desserts) and poor nutritional status from preconception to pregnancy, highlighting the need for interventions to establish healthy eating habits pre-conception.

\subsection{Assessment and Rating Officers Influencing Food Environments in Early-Childhood-Education and Care (ECEC)}

\section{Ros Sambell, Naomi Saint, Leesa Costello, Amanda Devine and Ruth Wallace Edith Cowan University, Floreat, Australia}

The quality of food provided in ECEC settings can dramatically influence health and developmental outcomes among children. Ultimately, monitoring ECEC food quality is the responsibility of jurisdictional regulatory authorities, but inconsistency in assessment protocols is common. Hence, this study investigated a) education undertaken by Lead Assessors (LAs) to train Authorised Officers (AOs) in ECEC food quality assessment and b) how AOs assess/rate services. Interviews with LAs $(n=8)$ from each jurisdiction and a survey with $\mathrm{AOs}(n=20)$ from one jurisdiction were transcribed and thematically analysed. LAs recognised the importance of nutrition and sought: expert advice, resources and guidance to foster compliant food environments through a Quality Improvement Plan (QIP); monitoring and compliance feedback; a 'toolbox' of questions for onsite use; guidance for observing embedded practice; and support to provide advice for meaningful engagement/communication with families, encouraging observation of food safety and food allergy management. In comparison, AOs reviewed and authenticated menus; service nutrition promotion; children self-serving at mealtimes; reference to Dietary Guidelines, ACECQA, and Get Up and Grow resources. It is recommended that a report card be developed as a mechanism for shared learning between LAs and AOs and to facilitate a QIP process and support consistent service rating in ECEC.

\subsection{Is the Diet Quality of Younger and Older Australian Women of Reproductive Age Different?}

\section{Nahal Habibi ${ }^{1,2}$, Katherine M. Livingstone ${ }^{3}$, Suzanne Edwards ${ }^{4}$ and Jessica A. Grieger ${ }^{1,2}$}

1 Adelaide Medical School, Faculty of Health and Medical Sciences, University of

Adelaide, Adelaide, Australia

2 Robinson Research Institute, University of Adelaide, Adelaide, SA, Australia

3 Institute for Physical Activity and Nutrition, School of Exercise and Nutrition Sciences,

Deakin University, Geelong, Australia

4 Adelaide Health Technology Assessment, University of Adelaide, Adelaide, Australia

Reproductive years are a major part of women's lifespan, and a healthy diet is critical for reproductive health. While more women are giving birth later in life, dietary differences of younger and older reproductive-aged women have been rarely assessed. We aimed to compare younger (19-35 y) and older (35-50 y) reproductive-aged women in three major dietary domains: The Australian Dietary Guidelines; the Acceptable Macronutrient Distribution range for protein, carbohydrates, and fat; and the Dietary Guideline Index (DGI). Data of 2323 women aged 19-50 years were extracted from the 2011-13 Australian 
National Nutrition and Physical Activity Survey. Regression models, adjusted for priori confounders, showed inadequate proportions of women meeting recommendations: $26 \%$ of women met the recommendations for fruit and meat and alternatives, and $<20 \%$ of women met the recommendations for vegetables, grains, and dairy with no difference between younger and older women. Although there was no difference between older and younger women in total DGI score (mean (SE) 75.6 (1.7) vs. 74.5 (2.5), $p>0.05$ ), older women had higher component scores in limiting saturated fat, consuming low-fat milk, and limiting adding salt during cooking. Nutrition education for women of reproductive age should be prioritised to improve their health and future generations.

\subsection{Breakfast Consumption Trends among Australian Children under 5 Years}

\section{Seon Park, Penny Love, Miaobing Zheng, Karen Campbell and Kathleen Lacy Deakin University, Geelong, Australia}

The breakfast meal has potential to improve daily dietary quality, particularly if it includes a range of food groups, including vegetables. Our study assessed average breakfast food and nutrient intakes at ages $1.5(n=369), 3.5(n=242)$, and $5.0(n=240)$ years using three 24-h dietary recalls from the Melbourne InFANT study. Tracking of food groups at breakfast was assessed by Pearson correlation of energy-adjusted food intake residuals across the three ages. Approximately $20 \%$ of total daily energy was consumed at breakfast across all ages. The main food groups consumed at breakfast were grains, milk/alternatives and discretionary foods, with increasing mean intakes and slight $(\mathrm{r}<0.3, p<0.05$; grains, discretionary) to moderate $(\mathrm{r} \geq 0.3, p<0.05$; grains, discretionary, milk/alternatives) tracking across the first 5 years. Vegetables were rarely consumed at breakfast ( $\leq 1.5 \mathrm{~g}$ mean intakes) at any age. Inconsistent tracking of fruit, meat/alternatives, and whole grains was observed over time. This study highlights the potential to enhance children's dietary intakes at breakfast. Given Australian children's discretionary and vegetable intakes do not meet recommendations, breakfast interventions may focus on practical strategies to increase vegetable intakes while reducing discretionary intakes.

\subsection{Economic Evaluations of Child Nutrition Interventions in Low-and Middle-Income Countries: Systematic Review and Quality Appraisal}

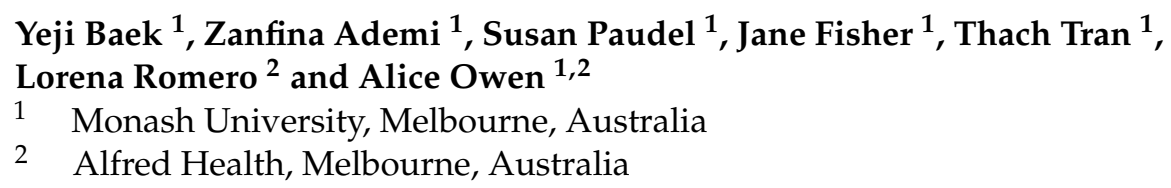

Economic evaluation is crucial for cost-effective resource allocation to improve child nutrition in low- and middle-income countries (LMICs). This systematic review aims to assess the quality of existing economic evaluations of child nutrition interventions in LMICs and synthesize the study characteristics and economic evidence. We searched nine databases, including MEDLINE, with the following concepts: economic evaluation, children, nutrition, and LMICs. All types of nutrition interventions, including stunting, wasting, micronutrient deficiency and overweight, were identified. We used the Drummond checklist for quality appraisal. Sixty-nine studies with diverse settings, perspectives, time horizons and outcome measures were included. Most studies used data from sub-Saharan Africa and South Asia and addressed undernutrition alone. Despite the heterogeneity of the included studies, $81 \%$ of included studies concluded that nutrition interventions were cost-effective or cost-beneficial. Regarding quality assessment, reporting the perspective of the analyses, justification of discount rates, and describing the role of funders and ethics approval were identified as areas needing improvement. The gaps in the quality of reporting could be improved by consolidated guidance on the publication of economic evaluations and the use of appropriate quality appraisal checklists. Strengthening the evidence base for child malnutrition across different regions is necessary to inform cost-effective investment in LMICs. 
3.20. Whose Responsibility Is Menu Planning in Early Childhood Education and Care Services-(ECEC)?

\section{Ros Sambell, Olga Martin, Kanita Kunaratnam and Ruth Wallace \\ Edith Cowan University, Floreat, Australia}

Food provision in ECEC settings is contentious. Research demonstrates that services aim to provide nutritious food for children, however, many do not comply with Australian Dietary Guidelines (ADG). The study aimed to determine directors' and food-coordinators confidence to plan nutritious menus' and explore roles and responsibilities around menuplanning. Participants $n=22$, attended a menu-planning/nutrition workshop, completed a survey assessing confidence to plan compliant menus. Each participant checked their menu against a menu-planning checklist and reviewed a nutrition policy; data relating to menu changes and perceived roles and responsibilities were captured. Pre-workshop, 64\% of participants were confident in planning an ADG-compliant menu; food-coordinators more confident $(73 \%)$ than directors $(55 \%)$ but not always allocated appropriate workloads. Directors (91\%) believed their role should focus on menu-planning. Participant-proposed menu changes included eliminating discretionary foods; reducing fat, sugar, and dried fruit in baking; increasing red meat and protein variety. All participants agreed that roles and responsibilities for menu-planning should be included in service policy; operational expectations were highlighted. These findings suggest that clearly outlined roles and responsibilities for food-coordinators and directors relating to menu-planning may improve menu quality at ECEC. Despite the reported confidence, menus continue to lack ADG compliance; therefore, ongoing training and support is essential.

\subsection{Menu Audit of Vegetable Offering in Primary School Canteens in Sydney, Australia}

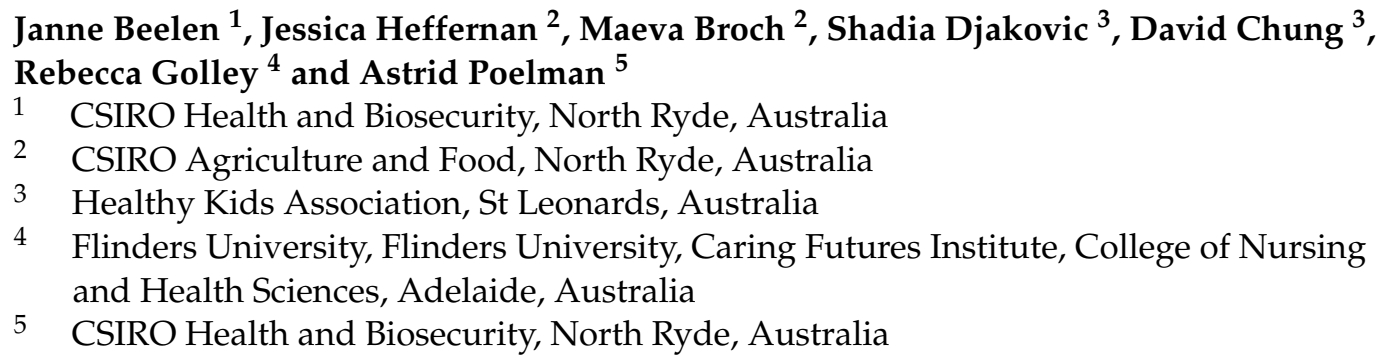

Considering most vegetables are consumed during dinner, school canteens could contribute to improving children's inadequate vegetable intake. This study developed and applied a systematic method to quantify the proportion and variety of vegetable-containing items on primary school canteen menus and examine the differences between schools of different socio-economic status, size, and type. Online canteen menus from 112 primary schools in Sydney were collected, and data extracted on total number of items and vegetablecontaining items across menu categories. Furthermore, data on the preparation type and variety were extracted. Differences in the proportions of vegetable items between socioeconomic status, school size and type were tested. On average, $80.4 \pm 34.0$ items were listed, with $30 \%$ of items containing vegetables. Most sandwiches $(60 \%)$ and hot foods $(54 \%)$ contained no vegetables. The variety of raw vegetables ( $4.9 \pm 1.8$ types) was greater than for cooked vegetables $(1.3 \pm 1.2$ types; $p<0.01)$. Limited differences were observed by socio-economic status and school type. Small schools offered fewer vegetable-containing items than large schools. Although canteen menus listed a large variety of items, only a third contained vegetables. The data of this study can be used to track changes and to develop new opportunities to increase the vegetable supply in schools. 
3.22. Who Are the Fussy Eaters? A Systematic Review of Children's Characteristics Associated with Food Fussiness

\section{Jookyeong Lee, Georgie Russell and Russell Keast \\ Deakin University, Geelong, Australia}

Children's biological characteristics are a crucial element in understanding the mechanisms and pathways of their eating behaviours and development. However, the role of children's biological characteristics in food fussiness has not been systematically examined. The aim of this review was to search, collate and summarize the evidence on associations between food fussiness and biological characteristics in children's fussy eating behaviours. A systematic review was performed with 2 main search concepts, food fussiness and biological characteristics, in children under 18 years old using 4 databases. A total of 46 articles were included. Selected articles ranged from medium-high to high quality. Children's food fussiness was associated with diverse biological characteristics including age, sex, temperament, heritability, birth weight, sensory sensitivity, eating rate, leptin level and chemosensory receptor genes. Most evidence focused on direct associations rather than indirect associations by examining covariates or mediators. A range of different measures were used for food fussiness assessment, which may be a potential cause of inconsistency in the outcomes of interest. This review has highlighted that many biological characteristics are related to food fussiness, yet there is inadequate attention directed to understanding mechanisms and pathways linking the biological characteristics with food fussiness in children.

3.23. Anthropometric Status of Indigenous Australian Infants and Young Children in the Gomeroi Gaaynggal Cohort

\section{Oyepeju Onifade ${ }^{1}$, Tracy Schumacher ${ }^{2}$, Megan Rollo ${ }^{3}, \mathrm{Kym} \mathrm{Rae}^{4}$ and Kirsty Pringle 5 \\ 1 School of Medicine and Public Health, University of Newcastle, Callaghan, Australia \\ 2 Department of Rural Health, School of Medicine and Public Health, Faculty of Health and Medicine, University of Newcastle, Tamworth, Australia \\ 3 School of Health Sciences, University of Newcastle, Callaghan, Australia \\ 4 Mater Research Institute, South Brisbane, Australia \\ 5 School of Biomedicine and Pharmacy, University of Newcastle, Callaghan, Australia}

The first 1000 days of life are a significant period for physical growth and development. The aim of this study was to assess the growth pattern of infants and young children in a cohort of Indigenous Australian children. Anthropometric status was assessed using the World Health Organisation (WHO) child growth standards as reference. Anthropometric measurements including weight, height and subscapular skinfold thickness were obtained by level-1-certified anthropometrists. The anthropometry indicators, weight-for-age (WFA), height-for-age (HFA), length-for-age (LFA), weight-for-height (WFH), BMI-for-age and subscapular skinfold-for-age were assessed. Children within \pm 2 standard deviations (SD) of the WHO median for the relevant indicator were categorised as within the normal range while those below 2SD were categorised as low. Anthropometric data were obtained from 127 independent children. The mean z-scores of LFA and BMI-for-age were $-0.15 \pm 1.16$ and $0.39 \pm 1.28$, respectively The prevalence of low HFA, low WFH, and low BMI-for-age were $5.9 \%, 6.2 \%$, and 3.5\%, respectively. Findings from this study show that although some of the anthropometric indicators were shifted to the right of the WHO curve, the majority of Indigenous Australian children in the Gomeroi gaaynggal cohort have a normal anthropometric status. We recommend that factors influencing anthropometric status should be investigated in future studies. 
3.24. Peer-Supported Lifestyle Interventions on Body Weight, Energy Intake and Physical Activity in Adults: A Systematic Review and Meta-Analysis

\section{Siew Lim ${ }^{1}$, Wai Kit Lee ${ }^{1}$, Andy Tan ${ }^{1}$, Mingling Chen ${ }^{1}$, Chau Tay ${ }^{1}$, Surbhi Sood ${ }^{1}$, Stephanie Pirotta ${ }^{1}$, Lisa Moran ${ }^{1}$, Meena Daivadanam ${ }^{2}$, Ljoudmila Busija ${ }^{3}$, Helen Skouteris ${ }^{1}$, Mamaru Awoke ${ }^{1}$ and Briony Hill ${ }^{1}$ \\ 1 Monash Centre for Health Research and Implementation, Clayton, Australia \\ 2 Department of Food, Nutrition and Dietetics, Uppsala University, Sweden \\ 3 School of Public Health and Preventive Medicine, Monash University, \\ Clayton, Australia}

There is an increasing interest in peer interventions in the management of chronic conditions, but evidence on peer interventions for body weight is lacking. The aim of this study was to examine the efficacy of peer interventions on body weight, energy intake and physical activity in adults. Interventions delivered by peer (lay member that participants identify with) were included. We searched fourteen databases. The outcomes were combined in the meta-analysis using the inverse variance random-effects model. From 2435 articles, 65 articles were included in the systematic review and meta-analysis $(n=15,673)$. Peer interventions resulted in significant reduction in weight [MD $-1.05 \mathrm{~kg}$; $95 \%$ confidence interval $(\mathrm{CI})-1.68,-0.43 ; 95 \%$ prediction interval $(\mathrm{PI})-3.25,1.14 ; 28$ studies; 7142 participants], BMI (MD $-0.24 \mathrm{~kg} / \mathrm{m}^{2} ; 95 \% \mathrm{CI}:-0.44,-0.04 ; 95 \%$ PI $-0.92,0.45$; 25 studies; 6672 participants), waist circumference (MD $-0.75 \mathrm{~cm}$; $95 \%$ CI: $-1.29,-0.21$; 95\% PI $-1.36,-0.14 ; 12$ studies; 4280 participants) and significant increase in physical activity (SMD 0.20; 95\% CI: 0.09, 0.32; 95\% PI - 0.46, 0.86; 41 studies; 10,778 participants), with no significant effect on energy intake. This study suggests that peer interventions are effective in reducing waist circumference, but further research is needed to confirm its effect on other obesity-related outcomes.

\subsection{A Clinical Cross-Sectional Feasibility and Acceptability Study of MRI and Dietary Assessments in Adults with Multiple Sclerosis}

\section{Olivia C. Wills and Yasmine Probst}

University of Wollongong, Wollongong, Australia

Multiple sclerosis is a chronic, neurological condition. Studies using objective measures of MS disability and in-depth dietary assessment tools are limited. This study aimed to explore the feasibility and acceptability of collecting MRI measures of disability at the same time point as measures of dietary intake. Structural and diffusion brain MRI scans and repeated 24-h dietary recalls were undertaken within the same week. Images were converted using dcm2niix and analysed using FreeSurfer software. Dietary profiles were developed using the multiple source method, and T1 and T2 brain lesion count and volume were assessed independently by two radiologists. In total, 15 participants consented, and 13 were included in the analysis. The recruitment rate was $47 \%$ and the eligibility rate was 91\%. MRI scans took on average $36 \mathrm{~min}$, collected 11,652 (IQR 2388) images and lesion counts ranged from 9 to 39 , primarily found in the periventricular, occipital and temporal brain regions. Diet profiles took a median time of $38.5 \mathrm{~min}$, and participants recalled between 12 and 38 different food items. The feasibility and acceptability of collecting MRI brain scans at the same time point as dietary profiles in adults with MS was confirmed. The burden of the protocol should be considered prior to upscaling.

3.26. Ethnic Differences in Response to Lifestyle Intervention for the Prevention of Type 2 Diabetes in Adults: A Systematic Review and Meta-Analysis

Mingling Chen ${ }^{1}$, Lisa J. Moran ${ }^{1}$, Cheryce L. Harrison ${ }^{1}$, Gebresilasea Gendisha Ukke ${ }^{1}$, Surbhi Sood ${ }^{1}$, Christie J. Bennett ${ }^{2}$, Mahnaz Bahri Khomami ${ }^{1}$, Pilvikki Absetz ${ }^{3}$, Helena Teede ${ }^{1}$ and Siew Lim ${ }^{1}$

1 Monash Centre for Health Research and Implementation, Monash University, Clayton, Australia 
2 Department of Nutrition, Dietetics and Food, School of Clinical Sciences,

Monash University, Notting Hill, Australia

3 Faculty of Social Sciences, Tampere University, Tampere, Finland

The risk of type 2 diabetes mellitus (T2DM) varies by ethnicity, but ethnic differences in response to diabetes prevention interventions remain unclear. This systematic review and meta-analysis assessed ethnic differences in the effects of lifestyle interventions on T2DM incidence, glycaemic outcomes (fasting glucose, 2-h glucose, HbA1c), anthropometric measures (weight, BMI, waist circumference) and lifestyle behaviours (physical activity, energy intake, energy from fat, fibre intake). MEDLINE, EMBASE and other databases were searched (to June 2020) for randomized and non-randomized controlled trials on lifestyle interventions (diet and/or physical activity) in adults at risk of T2DM. Ethnicity was categorized into European, South Asian, East and Southeast Asian, Middle Eastern, Latin American and African groups. Forty-four studies $(n=18,722)$ were included in the meta-analyses. Overall, lifestyle interventions resulted in significant improvement in T2DM incidence, glycemic outcomes, anthropometric measures, physical activity and energy intake (all $p<0.01$ ). Significant subgroup differences by ethnicity were found for 2-h glucose, weight, BMI and waist circumference (all $p<0.05$ ) but not for T2DM incidence, fasting glucose, $\mathrm{HbA} 1 \mathrm{c}$ and physical activity (all $p>0.05$ ). Few studies in non-European groups reported dietary intake. Lifestyle interventions in different ethnic groups may have similar effects in reducing the incidence of T2DM, although this should be confirmed in further studies.

\subsection{Dietary Patterns and Non-Communicable Disease Biomarkers: A Systematic Review and Network Meta-Analysis}

\section{Shuang Liang ${ }^{1}$, Jovana Mijatovic ${ }^{1}$, Ang Li ${ }^{2}$, Reeja Nasir ${ }^{1}$, Clemence Toniutti ${ }^{1}$, Kim Bell-Anderson ${ }^{3}$, Michael Skilton ${ }^{1,4}$ and Fiona $\mathrm{O}^{\prime}$ Leary ${ }^{1}$ \\ 1 Faculty of Medicine and Health, The University of Sydney, Sydney, Australia \\ 2 Melbourne School of Population and Global Health, The University of Melbourne, \\ Melbourne, Australia \\ 3 School of Life and Environmental Sciences, The University of Sydney, Australia \\ 4 Sydney Institute for Women, Children and their Families, Sydney Local Health District, Sydney, Australia}

Diet is an important risk factor for non-communicable diseases (NCDs), although comparisons of different dietary patterns have been limited. We conducted a systematic review of randomized trials with network meta-analysis to determine dietary patterns' comparative effects on biomarkers of NCDs. In total, 68 articles were included in a random effects network meta-analysis comparing (Mean Difference) and ranking (SUCRA) 11 dietary patterns (Mediterranean, DASH, Paleo, dietary guidelines-based, low GI/GL, plant-based, low-fat, low-carbohydrate high-fat, traditional Mexican, high GI/GL and Western habitual). Relative to the Western habitual diet, the Mediterranean, DASH, dietary guidelines-based, plant-based and low-fat diets reduced apoB, total and LDL cholesterol (MD range: -0.11 to $-0.07 \mathrm{~g} / \mathrm{L},-0.36$ to $-0.24 \mathrm{mmol} / \mathrm{L}$, and -0.29 to $-0.17 \mathrm{mmol} / \mathrm{L}$, respectively, all $p<0.05$ ); Paleo, plant-based and dietary-guidelines-based diets reduced HOMA-IR ( -0.95 to -0.35$)$. The Mediterranean diet was best for lipids (total/LDL/HDL cholesterol, triglycerides, apoB and apoA1, average SUCRA 63.1\%); the low-carbohydrate high-fat diet was best for glycaemic control (glucose, insulin and HOMA-IR, SUCRA 66.4\%); and the Paleo diet was best at reducing inflammatory biomarkers (CRP and interleukin-6, SUCRA: 87.0\%). While no dietary pattern ranked uniformly highest across all biomarkers of NCDs, the Paleo diet had the highest all-outcomes-combined ranking $(63.1 \%)$ and warrants further consideration for NCD prevention. 
3.28. Efficacy of a Dietitian-Led Very Low-Calorie Diet (VLCD)-Based Model of Care to Facilitate Weight Loss for Obese Patients Prior to Elective, Non-Bariatric Surgery

\author{
Sally Griffin ${ }^{1,2}$, Lynda Ross ${ }^{1}$, Matthew Burstow ${ }^{3}$, Ben Desbrow ${ }^{4}$ and \\ Michelle Palmer ${ }^{1,4}$ \\ 1 School of Exercise and Nutrition Sciences, Queensland University of Technology, \\ Brisbane, Australia \\ 2 Department of Nutrition and Dietetics, Queensland Health, Logan Hospital, Australia \\ Division of Surgery, Logan Hospital, Queensland Health, Australia \\ 4 School of Allied Health Sciences, Griffith University, Gold Coast, Australia
}

Elective surgery in obese adults carries higher risk of post-operative infection and prolonged hospital stays. We aimed to determine the efficacy of a dietitian-led very lowcalorie diet (VLCD)-based model of care in achieving weight loss for obese patients prior to surgery. This mixed-methods study included a medical chart audit of patients referred to a VLCD-based model over 23 months, as well as a survey of recently treated patients and surgeons who utilised the model. Weight loss targets were set by surgeons, and the dietitian prescribed VLCD-based treatment. Efficacy was determined as weight loss considered sufficient for surgery, clinical safety, feasibility, and stakeholder value. Seventy-eight eligible patients (45 \pm 13 years, $90 \% \mathrm{~F}$, BMI $44.3 \pm 6.2 \mathrm{~kg} / \mathrm{m}^{2}$ ) demonstrated significant weight loss of $7.4 \pm 5.3 \%$ body weight $(p<0.001)$. Most patients $(70 \%, n=50 / 71)$ achieved sufficient weight loss to proceed to surgery. Surgeons reported easier $(83 \%, n=10 / 12)$ and shorter $(75 \%, n=9 / 12)$ operations, and all recommended the model. All surveyed patients $(n=24)$ reported satisfaction with their experience. A dietitian-led VLCD-based model achieved sufficient weight loss to facilitate elective surgery for most patients. The approach was feasible, highly valued by patients and surgeons, with perceived surgical benefits.

\title{
3.29. Diabetes-Related Sweet Taste Function Alterations in Adults: A Systematic Review
}

\author{
James Ohanian ${ }^{1}$, Sze-Yen Tan ${ }^{1,2}$, Caitlyn Hack ${ }^{1}$, Cindy Yu ${ }^{1}$, Isabella Rennick ${ }^{1}$, \\ Marina Dezan ${ }^{1}$, Nicole Mott ${ }^{1}$, Rebecca Manibo ${ }^{1}$ and Robin M. Tucker ${ }^{3}$ \\ 1 School of Exercise and Nutrition Sciences, Deakin University, Geelong, Australia \\ 2 Institute for Physical Activity and Nutrition (IPAN), Deakin University, \\ Burwood, Australia \\ 3 Department of Food Science and Human Nutrition, Michigan State University, \\ East Lansing, United States
}

Maintaining optimal blood glucose is the goal of diabetes mellitus (DM) management. Simple carbohydrates, such as sugars, raise blood glucose rapidly after ingestion; thus, intake should be limited among people with DM. However, sugars add sweetness and increase the palatability of food and are difficult to avoid. DM triggers several physiological changes that are closely associated with human taste function. Therefore, the aim of this systematic review was to investigate if sweet taste impairment occurs in DM. A systematic search was conducted in four electronic databases, and 18 studies that reported the sweet taste function of people with diabetes were included. Almost all studies reported significantly lower sweet taste sensitivity (i.e., higher detection or recognition thresholds) and reduced perceived sweetness in people with DM. Only two studies examined sweet hedonics (liking or preference) in DM, and findings were conflicting. Sweet taste impairment was negatively associated with age and positively with BMI, waist circumference and peripheral neuropathy (but not retinopathy, nephropathy or autonomic neuropathy). Sweet taste impairment was not associated with glycaemic control, length of DM diagnosis or medication use. The dietary and health implications of sweet taste sensitivity impairment in DM should be investigated in future studies. 


\subsection{The Effects of Diet Quality on Protein Leverage and Energy Intakes}

\section{Amanda Grech, Stephen Simpson and David Raubenheimer}

The University of Sydney, Sydney, Australia

Both fats and carbohydrates have been proposed to have played a role in the global obesity crisis. However, humans possess a strong protein appetite, which drives food intake. When the macronutrient contribution of protein is reduced, fat, carbohydrate, and, consequently, total energy are overconsumed. We hypothesize that the underlying quality of the food composition modulates the protein leverage effect. We applied the Geometric Framework for Nutrition to a large national nutrition survey to examine the effects of different dietary patterns on protein dilution and energy intakes. Protein dilution predicted overall higher energy intakes. However, diets rich in refined grains and added sugars were leveraged the most strongly, with energy intakes reaching up to $5000 \mathrm{~kJ}$. Intrinsic carbohydrates were leveraged by protein differently, contributing at most $2000 \mathrm{~kJ}$ to total energy intake and were associated with a comparatively higher proportion of energy from protein, dietary fibre and lower total energy intakes, even when combined with fat. The results demonstrate the most obesogenic dietary patterns were protein-dilute diets, including refined starch, added sugars and fats and low in dietary fibre. Here, we gain novel evidence that demonstrates protein dilution is key to higher energy intake for highly processed, Western diets.

3.31. The Impact of Meals with Varying Levels of Anti-Inflammatory Compounds on Postprandial Circulating Markers of Inflammation in Overweight Adults Aged 50-75 Years: A Pilot Study

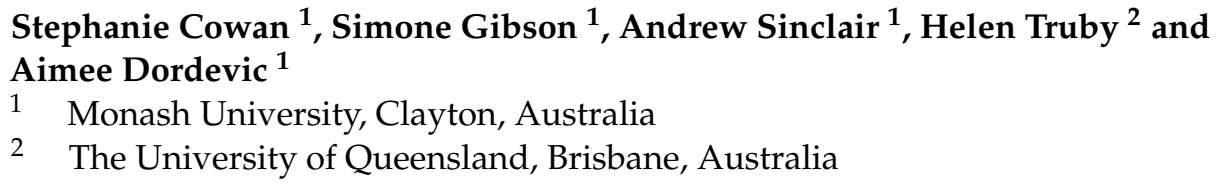

Subclinical inflammation is the underlying pathology of chronic metabolic diseases. While unhealthy dietary patterns are clearly linked to an inflammatory state, the acute impact of single meals remains unclear. This randomised, crossover pilot study aimed to determine whether three isocaloric meals with either pro-inflammatory, neutral or antiinflammatory components differentially affected circulating levels of inflammatory markers in 12 overweight and obese adults ( $\geq 50$ and $\leq 75$ years). Fasting and postprandial blood samples were collected over five hours and analysed for plasma levels of IL-6, IL-1 $\beta$, TNF- $\alpha$ and IL-10. Incremental areas under the curve for all markers were not significantly different between the three meals ( $p>0.05$ for all). There was a trend for IL- 6 to increase with time in all three meals. IL-1 $\beta$, TNF- $\alpha$ and IL-10 displayed a high degree of variability within and between participants, which possibly obscured any small meal-induced responses that may have occurred. Meals that differed in anti-inflammatory compound composition did not differentially impact circulating inflammatory markers in older, overweight adults. The high inter- and intra-individual variability of circulating cytokines suggests that future research should consider other markers that are more sensitive to nutrient flux when measuring postprandial metabolic inflammation.

3.32. An Insight into the Mechanism Underlying the Inhibition of Starch Hydrolysis by (Poly)phenols: Direct $\alpha$-Amylase Inhibition and/or Starch Complexation

Rizliya Visvanathan, Michael J. Houghton, Elizabeth Barber and Gary Williamson Nutrition, Dietetics and Food, Monash University, Notting Hill, Australia

High postprandial glycaemic excursions are an important factor contributing to the development of insulin resistance and type 2 diabetes (T2D). (Poly)phenol consumption is associated with a reduced risk of T2D, partly attributed by $\alpha$-amylase inhibition to reduce starch digestion. We present a new sensitive method to determine human $\alpha$-amylase inhibition using maltoheptaoside (Mal-7) as substrate with direct chromatographic product 
quantification, compared with amylose/amylopectin hydrolytic products estimated using 3,5-dinitrosalicylic acid (DNSA). The pharmaceutical drug acarbose exhibited similar $\mathrm{IC}_{50}$ values with Mal-7, amylopectin or amylose as substrates, whilst varied results were observed for the polyphenols, epigallocatechin gallate (EGCG), quercetagetin and punicalagin. The polyphenols weakly inhibited Mal-7 (<50\% inhibition) hydrolysis compared to amylose/amylopectin. (Poly)phenols may inhibit $\alpha$-amylase through (1) direct action on the enzyme and/or (2) starch-(poly)phenol complexation. EGCG and punicalagin inhibited $\alpha$-amylase mainly through starch complexation, whereas quercetagetin additionally binds to the $\alpha$-amylase active site. The new method using Mal-7 as the substrate is superior for determining direct $\alpha$-amylase inhibition, whilst the conventional DNSA method, using native starch as substrate, detects both inhibitory mechanisms. Accurately identifying (poly)phenols that inhibit $\alpha$-amylase and their mode of inhibition could aid in developing dietary strategies focused on reducing postprandial glycaemia.

\subsection{A Systematic Literature Review on the Effect of Lactoferrin Supplementation on Inflammation, Immune Function, and Respiratory Tract Infections}

\section{Bronwyn S. Berthon, Lily M. Williams, Evan J. Williams and Lisa G. Wood} Priority Research Centre for Healthy Lungs, Hunter Medical Research Institute, University of Newcastle, Newcastle, Australia

Lactoferrin (Lf) is a glycoprotein found in human and bovine milk with anti-microbial and immune modulating properties. This review examined the effect of Lf supplementation on inflammation, immune function, and respiratory tract infections (RTIs) in humans. Articles published up to December 2020 were selected from Medline, EMBASE, and CINAHL with data extraction and quality assessment completed using standardised tools. In total, 25 articles ( $n=2329$ subjects) were included, with 20 studies $(80 \%)$ in adults. Lf reduced systemic inflammation in eight studies $(n=13,61 \%)$. Immune function improved in six studies $(n=8,75 \%)$. Lf reduced RTI outcomes in six studies $(n=10,60 \%)$, with decreased incidence and either decreased frequency or duration of RTI's in at least $30 \%$ of studies. In meta-analyses, Lf reduced IL-6, but not CRP or natural killer cell cytotoxicity, and Lf reduced RTI incidence in infants and children, but not adults. This review found that Lf supplementation is safe in both adults and children. Doses of $200 \mathrm{mg} / \mathrm{d}$ reduce systemic inflammation, and formulas containing 35-833 mg/d Lf reduce the incidence of RTIs in infants and children, suggesting improved immune function. Future research is required to determine optimal dosing and identify populations most likely to benefit from Lf supplementation.

\subsection{The Mediterranean Diet and Multiple Sclerosis Disease Progression: Disability and Relapse Rate}

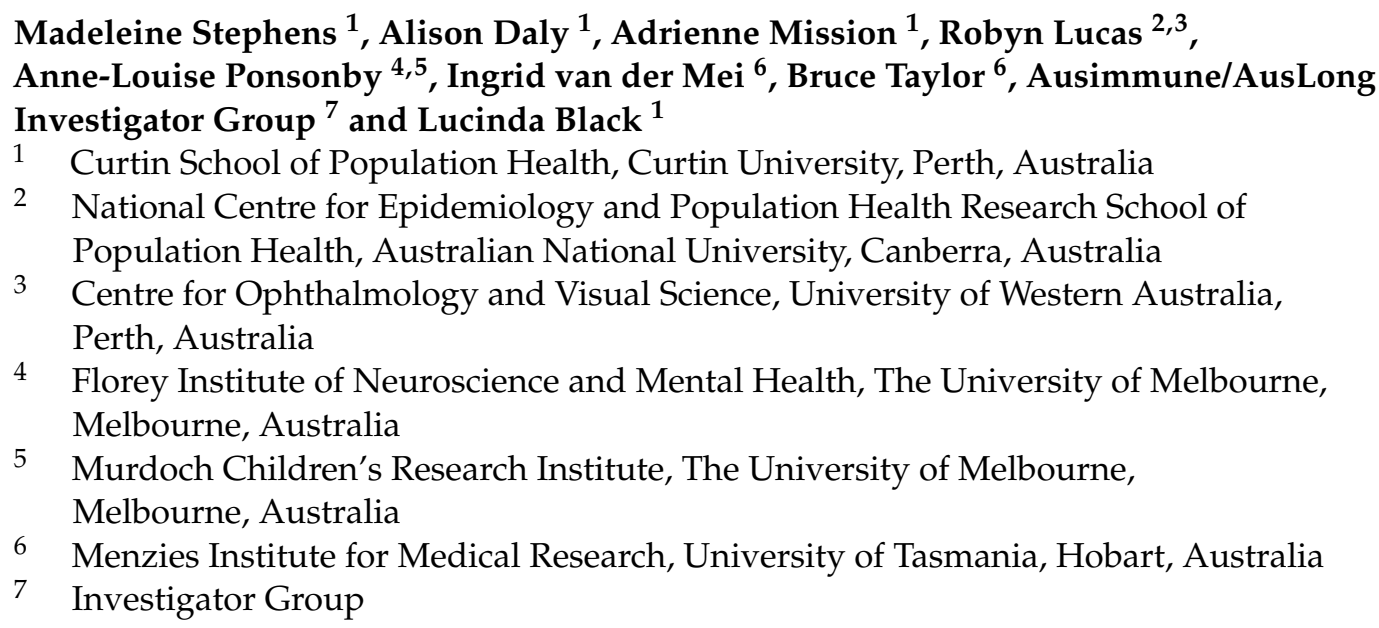


We previously showed that a higher Mediterranean diet score, including unprocessed red meat, is associated with reduced risk of central nervous system demyelination. We tested associations between the Mediterranean diet, disability and relapse rate in people with multiple sclerosis (MS). We used data from case participants of the 2003-2006 Ausimmune Study, along with data from the 5- and 10-year reviews ( $n=264, n=225, n=209$, respectively). Dietary intake data were collected using a food frequency questionnaire. We calculated the alternate Mediterranean diet score (aMED), energy-adjusted using the residual method. We used negative binomial analysis (mixed effects) to estimate Incidence Rate Ratios (IRR) for the Expanded Disability Status Scale (EDSS), and Cox regression to estimate the Hazard Ratio (HR) for relapse rate. We found no significant associations between the aMED and EDSS. There was a significant interaction between the aMED and $\mathrm{BMI}$ in relation to the relapse rate $(p<0.0001)$. With lower BMI, the aMED had a mitigating effect on relapse rate; as BMI increased, the effects of the aMED on the relapse rate attenuated. Our findings suggest the aMED is related to relapse rate, with a protective effect of a Mediterranean diet on relapse rate only when the BMI is lower.

\subsection{Social Network Analysis (SNA) of Nutrition Information Relating to the Management of Multiple Sclerosis}

\section{Emiliana Saffioti and Yasmine Probst}

University of Wollongong, Wollongong, Australia

Social media has changed the communication of health information; however, it is associated with quality concerns and poor reliability. People with Multiple Sclerosis, are reported to struggle with nutrition information online, yet the complexity of this information remains under-explored. We aimed to examine key sources, the accuracy and the acceptability of nutrition information related to MS management within social media platforms. This explanatory sequential, mixed-methods study performed SNA within YouTube and Twitter using NodeXL and further YouTube quantitative and qualitative content analyses. SNA metrics, QUEST scores and key themes were synthesised using Kumar et al.'s framework for detecting misinformation in online social networks. The endorsement of non-evidence-based diets for MS was prevalent within YouTube and Twitter. YouTube videos endorsing such diets were more likely to encourage viewers to purchase a product/service, be YouTube verified and have a higher subscriber count and lower QUEST score. Of all sources, doctors were most likely to endorse non-evidence-based diets. Nutrition misinformation relating to MS is prevalent in social networks, often influenced by financial motive/marketing. Doctors with a large online following may be inadvertent disseminators of misinformation, yet influential figures in MS care warranting further research to identify targets in the reduction of online misinformation.

\subsection{Communicating Health on Social Media: Industry Needs Survey}

\section{David Micallef ${ }^{1}$, Michaela Jackson ${ }^{1}$, Linda Brennan ${ }^{1}$, Annika Molenaar ${ }^{2}$, Eva Jenkins ${ }^{2}$, Shinyi Chin ${ }^{1}$ and Tracy A. McCaffrey ${ }^{2}$ \\ 1 School of Media and Communication, RMIT University, Melbourne, Australia \\ 2 Monash University, Notting Hill, Australia}

Health communication plays a significant role in health promotion and disease prevention. Our aim was to assess the learning needs of health professionals in relation to communicating health on social media. Participants were recruited through professional organisations, email lists and social media posts. A total of 77 participants completed the survey, with the majority identifying as health promotion professionals (38\%) or dietitians (36\%) working across multiple settings, including community health settings (54\%), hospitals $(40 \%)$ and private practices (29\%). An important goal for communication was 'tackling a specific area of health' (36\%) followed by 'supporting clients to manage their health outside of regular appointments' $(27 \%)$. Current clients were reached via in-person or virtual presentations $(90 \%)$, social media (83\%), direct emails (82\%) and telephone (81\%). Planning, selecting 
and developing targeted communications, as well as monitoring and evaluating were rated as being highly important $(>6 / 7)$. However, participants felt they were not highly skilled in these areas $(<4.9 / 7)$. When asked about the delivery mode for upskilling, infographics, short videos and factsheets were preferred ( $>90 \%)$. Whilst participants recognise the importance of communication skills in their roles, professional development activities should consider time constraints and cater to the needs identified in this survey.

\title{
3.37. Food and Nutrition Education and Training Requirements for Australian Aged Care Staff
}

\author{
Anneliese Twigg ${ }^{1}$, Karen Stafford ${ }^{1}$, Rivkeh Haryono ${ }^{2}$ and Sandra Iuliano ${ }^{3}$ \\ 1 Nutrition Australia, Melbourne, Australia \\ 2 Dairy Australia, Melbourne, Australia \\ 3 University of Melbourne, Melbourne, Australia
}

The Royal Commission into Aged Care Quality and Safety identified inadequate food provision and poor nutritional care and highlighted the requirement for nutrition education in the sector. We undertook an online survey and focus group assessments of aged care staff from a sample of metropolitan and regional facilities throughout Australia, to determine the food and nutrition education and training requirements and preferred mode of delivery. Among the 63 metropolitan and 37 regional/remote facilities, 89 responses to the survey were received, and 13 qualitative interviews were conducted. The main responders were managers (49\%), cooks, chefs and foodservices staff $(16 \%)$. The preferred modalities for education delivery were face-to-face $(71 \%)$ and online $(53 \%)$. Topics of interest were Nutrition for older adults (58\%) and texture-modified diets (55\%). Barriers to undertaking training included insufficient time allocated during paid work hours (34\%) and cost (34\%). Nutrition training and education to improve nutrition knowledge of staff may translate to the improvement in food quality and mealtime experience for residents and provide professional development, career advancement and staff retention strategies for the aged care workforce.

\subsection{Feasibility of Embedding Healthy Home Food Environment Messages into Recipes}

\author{
Alison Spence ${ }^{1}$, Melissa Burton ${ }^{1}$, Amber Kelaart ${ }^{2}$ and Kathleen Lacy ${ }^{1}$ \\ 1 School of Exercise and Nutrition Sciences, Deakin University, Geelong, Australia \\ 2 Nutrition Australia, Melbourne, Australia
}

Childhood diets are suboptimal, and a key influence on child diet is the home food environment, including parental feeding practices (for example, role modelling, child involvement, and repeated offering). A challenge in nutrition promotion is how to reach families with information about healthy home food environments as well as about healthy foods. This research aimed to explore the feasibility of embedding messaging on healthy home food environments into recipes. A website of recipes, nutrition and food skills resources was created for the food relief and health promotion sector in 2020. Short healthy home food environment messages were developed, reviewed by 4 parents and 2 health promotion organisations, and embedded into 47 of the website's recipes. Health professionals using those recipes provided feedback in semi-structured interviews. Of five participants who commented on the messages, all thought they were valuable and an appropriate way to share this information. Messages were perceived as subtle, simple, achievable and in line with the health professional's or organisation's existing practice and/or priorities. A suggested improvement was making the messages more upfront in recipes. This research suggests inclusion of healthy home food environment messages in recipes is feasible and warrants further assessment with parents and nutrition outcomes.

\subsection{Progressing the Development of a Food Literacy Questionnaire Using Cognitive Interviews}

Courtney Thompson ${ }^{1}$, Jean Adams ${ }^{2}$ and Helen A. Vidgen ${ }^{1}$

1 School of Exercise and Nutrition Science, Queensland University of Technology, Brisbane, Australia 
2 Centre for Diet and Activity Research (CEDAR), MRC Epidemiology Unit, University of Cambridge School of Clinical Medicine, Cambridge, United Kingdom

Food literacy is the knowledge, skills and behaviours needed to meet food needs and determine intake, and it is conceptualised as 11 components under the 4 domains of planning and managing, selecting, preparing, and eating. Previous measures of food literacy vary in their adherence to the conceptualisation. This study aimed to determine items for inclusion and exclusion in a food literacy item pool. Methods: Cognitive interviews were conducted on an item pool using think-aloud and verbal probing methods. Data were analysed for applicability, clarity, ambiguity, and logic. Australian residents over 18 years of age were recruited via Facebook residential groups $(n=20)$. Results: Of the original 116 items, 11 items had limited applicability, as living or food provisioning contexts did not apply to all participants. Thirteen items were unclear, as the purchasing, provisioning, or socialisation behaviours were vague. In addition, 32 items had lexical problems, with specific terms being confusing or ambiguous, and 11 items had logical problems, with items perceived to be addressing the same construct. Overall, 29 items were deleted, 31 were retained, and 56 were revised. An additional 84 items were developed to address participant feedback, resulting in a refined 171-item pool. Conclusion: This study progressed the development toward a comprehensive, validated food literacy questionnaire.

\subsection{Opportunities for Nutrition in Medical Education}

\section{Breanna Lepre ${ }^{1}$, Kylie J. Mansfield ${ }^{2}$ and Eleanor J. Beck ${ }^{1}$}

1 School of Medicine, University of Wollongong, and Illawarra Health \& Medical Research, Wollongong, Australia

2 Graduate Medicine, University of Wollongong, and Illawarra Health \& Medical Research, Wollongong, Australia

Poor diet is a leading cause of death worldwide. Doctors are well-placed to facilitate nutrition care, yet nutrition remains a low priority in medical education internationally. Consensus is required on nutrition competencies as a benchmark for education and on a regulatory framework to ensure implementation. This work is part of a larger project to develop a nutrition competency framework for medicine and aimed to examine medical practitioner perspectives of their role in nutrition care. Semi-structured interviews with medical practitioners from the United Kingdom and Australia explored perceptions of work roles, barriers and attitudes toward nutrition care and participant perceptions of required knowledge and skills. Participants acknowledged nutrition as an important component of medical care but recognised that they are currently ill-equipped to support such care, identifying limitations to the systems supporting integrated care. Many participants did not consider preventive nutrition, rather only broaching the subject once disease or a condition is present. Participants identified that nutrition sits within both a health promotion and medical/treatment model, but they currently work only within the latter. The description by participants of the paradigm shift required in patient care to embrace health promotion and disease prevention, and by proxy, nutrition, is also required in medical education.

3.41. An Investigation into the Suitability of Patient Education Materials Used by Dietitians for Older Adults with Type 2 Diabetes

\section{Claudia Goodman and Kelly Lambert}

University of Wollongong, Wollongong, Australia

Medical nutrition therapy is fundamental to the prevention and management of diabetes. Previous studies have consistently found that diabetes self-management patient education materials (PEMs) are not written in accordance with best practice health literacy principles. No research has evaluated the suitability of dietary materials, particularly those for older adults with diabetes. This knowledge may lead to developments to enhance the 
suitability of PEMs and facilitate optimal diabetes dietary management. This study evaluated PEMs commonly used by dietitians for older adults with diabetes by quantifying their readability, understandability, actionability and overall suitability. A purposive sample of PEMs were analysed using a readability calculator, the PEMAT, and the CCISS tool. Thirty materials were selected for analysis. Most materials (90\%) were written at a suitable readability level of $\leq$ Grade 8 . However, only $57 \%$ of materials were considered understandable, and $20 \%$ were considered actionable. Only $7 \%$ of materials were evaluated as exhibiting clear communication according to the CCISS tool. Areas for specific improvement included medical language, supporting visual aids, and actionable steps. These findings provide insights into areas requiring refinement and improvement. Future development of diabetes diet PEMs that include these amendments will better meet the learning needs of older adults with diabetes.

3.42. An Exploration of the Home Enteral Nutrition Service at a Tertiary Teaching Hospital in New South Wales

Maya M.Y. Young ${ }^{1}$, Rania R.L. Liaros ${ }^{2}$ and Anne-Therese A.M. McMahon ${ }^{1}$

1 Nutrition and Dietetics, Faculty of Science Medicine and Health, University of

Wollongong, Wollongong, Australia

2 Nutrition and Dietetics, St George Hospital, Kogarah, Australia

Australian home enteral nutrition (HEN) trends remain unknown, as a national framework and database is absent. This study explored a dietitian-led HEN service against the NSW Agency for Clinical Innovation (ACI) HEN Guidelines to develop recommendations for improvement. A two-year retrospective audit of HEN medical records $(n=252)$ and a cross-sectional experience survey of current HEN patients $(n=50)$ at an NSW tertiary teaching hospital was conducted. Audit analysis showed that HEN patients' mean age was 74.2 , with $91.6 \%$ of patients using oral nutrition support. Only $25.5 \%$ of patients were seen within the initial 1-to-2-week timeframe, and $91.5 \%$ of patients were reviewed every 3-6 months, as per ACI HEN Guidelines. The HEN services were rated positively, but the delivery (over the phone, face-to-face or a combination) influenced patient experience. These findings show inconsistencies with the ACI HEN Guidelines, as well as highlighting how the service delivery of HEN impacts patient experience; both findings warrant further research.

\subsection{A 10-Year Follow-Up of ISLHD Dietitians' Time Spent in the Hospital Setting: Has Anything Changed?}

\section{Vivien Hui In Cheung and Yasmine Probst}

University of Wollongong, Wollongong, Australia

The purpose of this study was to examine the current time spent by dietitians in the Illawarra Shoalhaven Local Health District (ISLHD) and comment on the changes over the past decade. This was an observational time and motion study across six healthcare facilities in the ISLHD between May and June 2021. Dietitians $(n=18)$ were identified as outpatient or inpatient dietitians. The results were recorded and compared with a study from the same district in 2008 to 2010 . Pearson's $\chi^{2}$ tests were conducted to compare data between outpatient and inpatient dietitians and between now and 10 years ago. Of the $376 \mathrm{~h}$ and $57 \mathrm{~min}$ of time recorded, a significant proportion of time was spent in indirect patient care (29.7-46.2\%), especially reviews of patient medical files and documentation (23.7-32.9\%). Compared to 10 years ago, more time was spent on indirect patient care, while less time was spent on direct patient care. Indirect patient care was found to dominate most of the dietitians' time. This was accompanied by decreased time for direct patient care and communication. Implementations of the electronic medical record in the district in the mid-2010s could correlate with this shift. The causes of the shift were unclear, and future studies should assess the corresponding changes in the dietetic care quality. 
3.44. The Association between Antenatal Diet Quality and Postpartum Depression in the MUMS Cohort

\author{
Megan L. Gow 1,2,3, Yei Wei Ingrid Lam ${ }^{4}$, Hiba Jebeile ${ }^{1,5}$, Maria E. Craig 1,3,5, \\ Daniella Susic ${ }^{3}$ and Amanda Henry ${ }^{2,3}$ \\ 1 Children's Hospital at Westmead Clinical School, The University of Sydney, \\ Westmead, Australia \\ 2 School of Women's and Children's Health, University of New South Wales, \\ Sydney, Australia \\ 3 Women's and Children's Health, St George Hospital, Kogarah, Australia \\ 4 Faculty of Science, The University of Sydney, Sydney, Australia \\ 5 The Institute of Endocrinology and Diabetes, The Children's Hospital at Westmead, \\ Westmead, Australia
}

Good antenatal nutrition is inconsistently reported to play a role in preventing postpartum depression. Therefore, this study aimed to investigate the association between diet quality during pregnancy and postpartum depression. Secondarily, this study investigated associations between (i) diet quality during pregnancy and depression during pregnancy and (ii) depression during pregnancy and postpartum depression. This study is a secondary data analysis of women $(n=73)$ participating in the Microbiome Understanding in Maternity Study (MUMS) Cohort in Sydney, Australia. The participants' diets were assessed during pregnancy using the Australian Eating Survey at Trimester 1 and 3, and depression was assessed using the Edinburgh Depression Scale at Trimester 1, 2, 3 and 6 weeks postpartum. Correlation coefficients were calculated in SPSS. There were no significant correlations between diet quality during pregnancy and depression scores postpartum or during pregnancy. Depression scores during pregnancy were associated with postpartum depression score (Trimester $1: \mathrm{r}=0.66$, Trimester $2: \mathrm{r}=0.69$, Trimester 3: $\mathrm{r}=0.67$; all $p<0.001)$. Overall, diet quality during pregnancy did not influence depression during pregnancy or early postpartum. However, depression scores during pregnancy were associated with postpartum depression score highlighting the importance of early screening for depression during pregnancy.

\title{
3.45. Mediterranean Diet in Relation to Cognitive Function: An Umbrella Review of Meta-analyses of Epidemiological Studies
}

\author{
Matina Kouvari ${ }^{1,2}$, Nathan M. D'Cunha ${ }^{1,2}$, Nikolaj Travica ${ }^{3}$, Wolfgang Marx ${ }^{3}$, \\ Domenico Sergi ${ }^{4}$, Manja Zec ${ }^{5}$ and Nenad Naumovski ${ }^{1,2}$ \\ 1 University of Canberra, Australia \\ 2 Functional Foods and Nutrition Research (FFNR) Laboratory, University of Canberra, \\ ACT, Australia \\ 3 Food and Mood Centre, Deakin University, IMPACT-The Institute for Mental and \\ Physical Health and Clinical Translation, School of Medicine, Barwon Health, \\ Geelong, Australia \\ 4 Department of Translational Medicine, University of Ferrara, Ferrara, Italy \\ 5 School of Nutritional Sciences and Wellness, University of Arizona, Tucson, \\ United States
}

Adherence to the Mediterranean diet (MD) may improve cognitive disorders. We aimed to conduct an umbrella review of the meta-analyses on MD and cognitive impairment. Searches were conducted in PubMed, Embase, and Cochrane database until August 2021, identifying relevant meta-analyses. Four meta-analyses examined the effect of MD on mild cognitive impairment $(\mathrm{MCI})(n=1$; studies included $=5$; participants $=24,274)$, cognitive impairment $(n=1$; studies included $=7$; participants $=8291)$ and Alzheimer's disease $(A D)$ incidence $(n=2$; studies included $=6$; participants $=138,471)$. The level of adherence to MD (high vs. low) resulted in a $17 \%$ lower risk of MCI (Relative Risk $(R R)=0.83,95 \%$ Confidence Interval $(95 \%$ CI) $(0.74,0.93)), 40 \%$ lower cognitive impairment risk $(\mathrm{RR}=0.60,95 \% \mathrm{CI}(0.43,0.83))$ while in $\mathrm{AD}$ individuals, the $\mathrm{RR}$ ranged from 0.63 
to 0.87 . In two meta-analyses a dose-response analysis was performed; per each two-point increase in MD score, the AD risk was significantly reduced by 13 to $14 \%$. Adherence to MD might favourably impact cognitive impairment and AD risk. Further research is essential to implement public health policy providing tailored strategies to increase adherence to $\mathrm{MD}$ in populations at high risk for cognitive impairment.

3.46. Effect of Omega-3 Supplementation on Self-Regulation in Typically Developing Preschool-Aged Children: Results of the Omega Kid Pilot Study a Randomised Double-Blind Placebo-Controlled Trial

Lauren A. Roach ${ }^{1,2}$, Mitchell K. Byrne ${ }^{3}$, Steven J. Howard ${ }^{4,5}$, Stuart J. Johnstone ${ }^{6}$, Marijka Batterham ${ }^{7}$, Ian M.R. Wright ${ }^{2,4,8}$, Anthony D. Okely ${ }^{2,4}$, Renate H.M.

de Groot ${ }^{9}$, Inge S.M. van der Wurff ${ }^{9}$, Alison L. Jones ${ }^{1,10}$ and Barbara J. Meyer ${ }^{2,11}$

1 School of Medicine, Faculty of Science, Medicine and Health, University of Wollongong, Wollongong, Australia

2 Illawarra Health and Medical Research Institute, University of Wollongong, Wollongong, Australia

3 College of Health and Human Sciences, Charles Darwin University, Darwin, Australia

4 Early Start, School of Health and Society, Faculty of the Arts, Social Sciences and Humanities, University of Wollongong, Wollongong, Australia

5 School of Psychology, Faculty of the Arts, Social Sciences and Humanities, University of Wollongong, Wollongong, Australia

6 Brain \& Behaviour Research Institute, School of Psychology, Faculty of the Arts, Social Sciences and Humanities, University of Wollongong, Wollongong, Australia

7 Statistical Consulting Centre, School of Mathematics and Applied Statistics, Faculty of Engineering and Information Sciences, University of Wollongong,

Wollongong, Australia

8 College of Medicine and Dentistry, James Cook University, Cairns, Australia

9 Conditions for Lifelong Learning, Faculty of Educational Sciences, Open University of The Netherlands, Heerlen, The Netherlands

10 Medical Education, Fiona Stanley and Fremantle Hospitals Group, Perth, Australia

11 School of Medicine, Molecular Horizons, Faculty of Science, Medicine and Health, University of Wollongong, Wollongong, Australia

The supplementation of omega-3 long-chain polyunsaturated fatty acids (n-3 LCPUFA) may enhance self-regulation (SR) and executive functioning (EF) in children of preschool age. The aim of the Omega Kid Study was to investigate the effect of n-3 LCPUFA supplementation on SR and EF in typically developing preschool-aged children. A double-blind, placebo-controlled pilot trial was undertaken; the intervention was 12 weeks long and consisted of $1.6 \mathrm{~g}$ of eicosapentaenoic acid and docosahexaenoic acid per day compared to a placebo. The HS-Omega-3 Index was assessed by capillary blood samples at baseline and post-intervention. In total, 78 children were enrolled and randomised to either the n-3 LCPUFA treatment $(n=39)$ or placebo $(n=39)$ group. At baseline, there were significant positive Spearman correlations found between the HS-Omega-3 index and both behavioural self-regulation and cognitive self-regulation $(r=0.287, p=0.015$ and $r=0.242$, $p=0.015$, respectively). Post-intervention, there was a significant three-fold increase in the HS-Omega-3 Index in the n-3 LCPUFA group $(p<0.001)$. There were no improvements in SR or EF outcome variables for the n-3 LCPUFA group post-intervention compared to the placebo group. Further research is required to target children with sub-optimal self-regulation who may benefit most from n-3 LCPUFA supplementation.

\title{
3.47. Diet Quality in Major Depressive Disorder: A Longitudinal Follow-Up Study
}

\author{
Alison Julien, Karen Charlton, Karen Walton, Theresa Larkin, Asmahan Elgellaie and \\ Susan Thomas \\ University of Wollongong, Wollongong/Keiraville, Australia
}


This study examined longitudinal relationships between the diet quality of participants with Major Depressive Disorder (MDD) and healthy controls (HC). Diet history and depressive symptoms (Depression Anxiety Stress Scale (DASS) were collected at baseline and follow-up, with an average of 13 months in between $(n=26 \mathrm{MDD}, n=21 \mathrm{HC})$. Diet quality was determined using the Australian Healthy Eating Index tool (Aust-HEI). At baseline, MDD participants had significantly lower Aust-HEI (Median $28.0( \pm 10.2)$ vs. 42.2 $( \pm 5.0) p<0.001)$ and higher DASS scores (Median $70.5( \pm 22.3)$ vs. $9.0( \pm 9.1) p<0.001)$ compared to HC. For MDD participants, both the Aust-HEI (Median $34.0( \pm 9.53) p=0.028)$ and DASS scores (median $54.2( \pm 29.7) p=0.006)$ improved significantly from baseline to followup. For all participants, a moderate inverse association was found between total DASS scores and Aust-HEI at baseline $(r=-0.565, p<0.001)$ and follow up $(r=-0.391, p=0.007)$. Participants with MDD had improvements in Aust-HEI over time $(p=0.014, p<0.001)$. These findings suggest that people with MDD, have poorer diet quality compared to HC and that both depression and diet improved simultaneously.

\subsection{Role of Cholecystokinin in Satiation and Regulation of Body Weight: A Systematic Review and Meta-Analysis}

\section{Andrew Warrilow, Murray Turner, Nenad Naumovski and Shawn Somerset Faculty of Health, University of Canberra, Bruce, Australia}

The aim of this study was to investigate the effects of cholecystokinin (CCK) or analogues of CCK on satiation and regulation of body weight in healthy humans. A systematic review of the literature was performed following the PRISMA 2020 guidelines in five electronic databases. A total of 1054 studies were found using the search terms, which was reduced to 15 studies suitable for inclusion. Ten studies measured the effect on satiation via subjective appetite ratings, with nine showing a significant positive influence on measures of satiety. Of the 13 studies measuring the effect on energy intake, 12 studies showed a decrease in energy intake. An analogue of CCK brought about an increase in satiation yet failed to produce any weight loss at 24 weeks. A meta-analysis of studies which infused CCK at lower 'physiological' doses found the group which combined CCK infusion with gastric distension produced a significant effect on satiation, whereas those which infused CCK alone did not. The present review found evidence that exposure to CCK results in an increase in satiation but no evidence for weight loss. At physiological doses, there is evidence that CCK has a greater effect on appetite when combined with stomach distension.

\subsection{Does Timing of Phytonutrient Intake Influence the Suppression of Postprandial Oxidative} Stress? A Systematic Literature Review

\section{Margaret Murray 1,2, Sophie Selby-Pham ${ }^{2}$, Beau Colton ${ }^{1}$, Louise Bennett ${ }^{2}$, Gary Williamson ${ }^{1}$ and Aimee L. Dordevic ${ }^{1}$ \\ 1 Department of Nutrition, Dietetics \& Food, Monash University, Australia \\ 2 School of Chemistry, Monash University, Australia}

Phytonutrients show potential to suppress the postprandial oxidative stress that occurs following typical Western-style meals. Due to the transience of phytonutrients in circulation, we hypothesise that timing of phytonutrient consumption, relative to the meal, may be important for oxidative stress suppression. Reviewed studies met the criteria: (1) healthy human adults; (2) phytonutrient consumed in solid form within $24 \mathrm{~h}$ of a challenge meal; (3) postprandial measurements of oxidative stress or antioxidants in blood; and (4) controlled study design. Compared with control, significant decreases in oxidative stress markers (lipid hydroperoxides, malondialdehyde, sNox2-dp, 8-iso-PGF2 $\alpha$, p47phox and Keap-1) were observed where the phytonutrient was consumed at the same time as the challenge meal $(n=4)$. Meanwhile, no effect on oxidative stress markers was observed where phytonutrients were consumed at the same time $(n=1)$, one hour before $(n=1)$, or the day before $(>12 h)(n=2)$ the challenge meal. While only a limited number of studies 
were included, oxidative stress suppression appeared effective when the phytonutrient was consumed at the same time as the challenge meal. Further research is required to confirm these observations and systematically optimise the effect of phytonutrient timing.

\title{
3.50. The Effect of Maternal Dietary Glycaemic Index on Offspring Metabolic Phenotype in Mice
}

Sophie Lucic Fisher ${ }^{1}$, Amanda Brandon ${ }^{2}$, Samantha Solon-Biet ${ }^{2}$ and Kim Bell-Anderson ${ }^{1}$

1 Charles Perkins Centre and School of Life and Environmental Sciences, The University of Sydney, Sydney, Australia

2 Charles Perkins Centre, School of Medical Sciences, The University of Sydney, Sydney, Australia

Carbohydrates are the most abundant macronutrient in the diet, their quality is critical for good health. The glycaemic index (GI) determines carbohydrate quality based on postprandial blood glucose, with low-GI foods associated with better metabolic health This study compared free-sugars with GIs from glucose (highest), sucrose (middle) and isomaltulose (lowest). C57BL/6 female mice were fed one of three sugar-based diets or an AIN93-G control. Pups continued on their mother's diet until 30 weeks. Body composition was determined by EchoMRI, food intake by BIODAQ and oral glucose tolerance tests (OGTT) performed. Preliminary results show that 30-week female highsugar mice were heavier, fatter and produced more insulin in response to the OGTT. In male mice, glucose-fed pups were heavier and all sugar-fed pups were fatter. Food intake was increased in sugar-fed pups. Liver fibrosis was observed in glucose (32\%)-, sucrose (38\%)- and isomaltulose (24\%)-fed mice. The results show female mice are more heavily affected at 30-weeks across all sugar diets with a higher body weight, percent fat, insulin response to an OGTT and increased food uptake. Male mice show less differences, but sugar still affects body composition, food intake and liver fibrosis. These findings show that free-sugars, regardless of their GI, can induce changes in body composition and risk of metabolic disease.

\subsection{Assessing the Health Effects of a High Resistant Protein Diet in Young Pigs-A Study Protocol}

\author{
Margaret Murray 1,2, Melinda Coughlan ${ }^{3,4}$, Francine Marques 5,6, \\ Sophie Selby-Pham ${ }^{2}$, Matthew Snelson ${ }^{3}$, Kirill Tsyganov ${ }^{5,7}$, Gary Williamson ${ }^{1}$, \\ Tong $\mathrm{Wu}^{2}$ and Louise Bennett ${ }^{2}$ \\ 1 Department of Nutrition, Dietetics \& Food, Monash University, Australia \\ 2 School of Chemistry, Monash University, Australia \\ 3 Department of Diabetes, Monash University, Melbourne, Australia \\ 4 Baker Heart \& Diabetes Institute, Melbourne, Australia \\ 5 Hypertension Research Laboratory, Monash University, Melbourne, Australia \\ 6 Heart Failure Research Group, Baker Heart and Diabetes Institute, \\ Melbourne, Australia \\ 7 Bioinformatics Platform, Monash University, Clayton, Australia
}

High-heat processed foods undergo chemical modifications that cause proteins to become resistant to enzymatic digestion. These resistant proteins reach the colon where they are fermented by gut microbiota. Excess resistant protein fermentation may be harmful to health via forfeited protein nutrition, altering gut microbial composition, and production of potentially harmful fermentation metabolites, such as phenols, indoles and amines. Dietaryresistant proteins are present in ultra-processed foods, processed forms of infant formulae, and 'meat' substitutes made from processed plant proteins. This study will determine the effects of a high-resistant protein diet on growth, gut microbiome, protein fermentation metabolites, and biomarkers of health status. Weanling pigs are fed a standard $(n=4)$ or resistant protein $(n=4)$ diet, which is approximately half as digestible and designed to enter the colon for microbial fermentation, for 4 weeks (both $21 \% \mathrm{w} / \mathrm{w}$ protein). Faecal 
samples are collected to assess the microbiome via $16 \mathrm{~S}$ rRNA gene bioinformatics. Blood samples are collected to measure circulating metabolites and biomarkers via metabolomics analysis. Pig weight and feed intake is monitored throughout the study. The results from this study will shed light on the mechanisms that link diets high in resistant proteins with potential adverse health outcomes.

\subsection{The Metabolisable Energy of Tree Nuts and Peanuts: A Systematic Review}

Cassandra Nikodijevic ${ }^{1}$, Yasmine Probst ${ }^{1,2}$, Sze-Yen Tan ${ }^{3}$ and Elizabeth Neale ${ }^{1,2}$

1 School of Medicine, Faculty of Science Medicine and Health, University of Wollongong, Wollongong, Australia

2 Illawarra Health and Medical Research Institute, University of Wollongong, Wollongong, Australia

3 Institute for Physical Activity and Nutrition (IPAN), School of Exercise and Nutrition Science, Faculty of Health, Deakin University, Burwood, Australia

Nuts are an energy-dense food, yet habitual consumption is not associated with weight gain. Body weight is determined by energy intake and energy expenditure. A proportion of the energy found within nuts remains encapsulated within cell walls and therefore cannot be digested. We conducted a systematic review to examine the metabolisable energy of tree nuts and peanuts (PROSPERO CRD42021252287). PubMed, MEDLINE, CINAHL, Cochrane, and Embase were searched to June 2021, using relevant controlled vocabulary and free text terms. Both in vitro and human studies (adults 18 years and older) were included. Quality appraisal was performed using the Academy of Nutrition and Dietetics Quality Criteria Checklist for human studies, and a modified Office of Health Assessment and Translation risk-of-bias tool for in vitro studies. Of the 10,490 non-duplicate articles on metabolisable energy found, 24 were included after title and abstract and full-text screening by two independent researchers. The metabolisable energy of nuts was found to be consistently lower than that predicted by Atwater factors for all investigated nut types (almonds, cashews, hazelnuts, pistachios, walnuts, and peanuts) due to lower lipid release, and this lower-than-expected metabolisable energy may explain the lack of associations between nut intake and body weight observed in the literature.

\subsection{Resistant Starch Supplementation Alters the Gut Microbiota and Intestinal Tight Junction Expression in Diabetic Mice}

\section{Matthew Snelson, Sih Min Tan, Cassandra De Pasquale and Melinda T. Coughlan Department of Diabetes, Monash University, Melbourne, Australia}

This study investigated the effects of resistant starch (RS) supplementation on the gut microbiota and intestinal homeostasis in diabetic mice. Six-week-old non-diabetic mice $(\mathrm{db} / \mathrm{h})$, diabetic mice $(\mathrm{db} / \mathrm{db})$ and $\mathrm{db} / \mathrm{db}$ mice on a regular chow diet supplemented with $15 \%$ RS (dbdb + RS) were maintained for 10 weeks. Ileum and jejunum sections were collected, RNA was extracted and cDNA was synthesised for quantitative gene expression analysis. Cecal digesta were collected for microbiota analysis by $16 \mathrm{~S}$ rRNA gene sequencing. Diabetes was associated with a reduction in relative expression of the tight junction protein Claudin-7 in both the jejunum and ileum (1.17 \pm 0.63 vs. $0.22 \pm 0.13$, $p<0.01$ and $1.19 \pm 0.61$ vs. $0.63 \pm 0.35, p<0.05$, respectively, $\mathrm{db} / \mathrm{h} \mathrm{vs} . \mathrm{db} / \mathrm{db})$, which was ameliorated with RS supplementation ( $0.22 \pm 0.13$ vs. $1.12 \pm 0.89, p<0.05$ and $0.63 \pm 0.35$ vs. $1.22 \pm 0.50, p<0.05$, respectively, $\mathrm{db} / \mathrm{db}$ vs. $\mathrm{db} / \mathrm{db}+\mathrm{RS})$. Between $\mathrm{db} / \mathrm{db}$ mice, RS favourably altered the microbiome, specifically an expansion of Verrucomicrobia, driven largely by the genus Akkermansia, and a contraction in Proteobacteria, driven by the sulphate reducing bacterial family Desulfovibrionaceae. These studies indicate that dietary RS may modify intestinal homeostasis via the alteration of the gut microbiota. 
3.54. Assessing Perceptions and Practices of Fitness Professionals in Relation to Body Acceptance and Weight Bias in Their Clients in Exercise Settings

\author{
Kanita Kunaratnam ${ }^{1}$, Cole Fox ${ }^{1}$ and Zali Yager ${ }^{2}$ \\ 1 ECU, Joondalup, Australia \\ 2 College of Arts and Education, Victoria University, Melbourne, Australia
}

Research has shown that weight stigma experienced by overweight and obese individuals within fitness facilities may be a psychological stressor which leads them to drop out of exercise regimes. We aimed to assess perceptions and practices of fitness professionals in relation to body acceptance and weight bias. One hundred and twenty-six fitness professionals completed an online survey assessing exercise information relayed to clients, thoughts towards body acceptance and confidence in reducing weight bias. Fitness professionals reported that both male and female clients experience weight stigma equivocally. The higher dropout of male clients was due to not being able to meet appearance expectations, whereas for female clients, this was in relation to not meeting performance expectations. Fitness professionals with an education level $\leq$ year 12 display higher body acceptance scores (mean score: $83.7 \pm 16.7)$ compared to tertiary educated fitness professionals (mean score: $63.5 \pm 22.3)(p=0.04)$. Fitness professionals aged 18-24 years had lower confidence promoting body acceptance (mean score: $58.1 \pm 25.5$ ) compared to those $\geq 35$ years (mean score: $77.1 \pm 14.9)(p=0.008)$. Efforts to promote exercise compliance and reduce dropout rates among clients in larger bodies should incorporate strategies to reduce weight stigma among fitness professionals and increase confidence in addressing these.

\title{
3.55. Appetitive Phenotype Trajectories: A Longitudinal Cohort Study of Early Origins and Influences across Infancy
}

\section{Georgie Russell ${ }^{1}$, Jessica Appleton ${ }^{2}$, Alissa J. Burnett ${ }^{1}$, Chris Rossiter ${ }^{2}$,} Cathrine Fowler ${ }^{3}$, Elizabeth Denney-Wilson ${ }^{1,2}$ and Elena Jansen ${ }^{4}$

1 Deakin University, Geelong, Australia

2 Susan Wakil School of Nursing and Midwifery, University of Sydney, Sydney, Australia

3 School of Nursing and Midwifery, Faculty of Health, University of Technology Sydney, Sydney, Australia

4 Division of Child \& Adolescent Psychiatry, Department of Psychiatry \& Behavioral Sciences, Johns Hopkins University School of Medicine, Baltimore, United States of America

Objective: The objective of the present study was to empirically describe appetitive phenotype trajectories in infancy and examine associations with infant and parent factors. Methods: In this longitudinal cohort study of Australian infants, parents completed three surveys approximately three months apart, beginning when the infant was $<6$ months. Appetitive traits were assessed with the Baby Eating Behaviour Questionnaire (BEBQ), and parent feeding practices with the Feeding Practices and Structure Questionnaire (FPSQ) infant and toddler version. Parent and infant characteristics were also collected. Groupbased trajectory modelling identified appetitive phenotype trajectories using the BEBQ. Multilevel modelling examined changes in feeding practices and child BMI z-score over time by appetitive phenotype trajectories. Results: There were 380 participants at time 1, 178 at time 2 and 154 at time 3 . Three appetitive phenotype trajectories were identified, labelled as follows: (1) food-avoidant trending towards low food approach (21.32\% of infants), (2) balanced (50.53\% of infants) and (3) high and continuing food approach $(28.16 \%$ of infants). Formula feeding, persuasive feeding and parent cognitions were related to infant appetitive phenotypes. Conclusion: Distinct appetitive phenotype trajectories emerge early in infancy and suggest that, for some infants, difficulties in self-regulating appetite emerges early in life. 


\subsection{A Transcriptomic Approach to Predicting Weight Loss Success}

Kaitlin Day ${ }^{1}$, Helen Truby ${ }^{2}$, Kay Nguo ${ }^{1}$, Melissa Southey ${ }^{3,4,5}$, Garun Hamilton ${ }^{6,7}$, Bradley Edwards ${ }^{8}$, Alan Young ${ }^{9,10}$, Denise O'Driscoll ${ }^{9,10}$ and Chiara Murgia ${ }^{11}$

1 Department of Nutrition, Dietetics and Food, Monash University, Melbourne, Australia

2 School of Human Movement and Nutrition Sciences, University of Queensland, Brisbane, Australia

3 Department of Clinical Pathology, Melbourne Medical School, Melbourne University, Melbourne, Australia

4 Cancer Epidemiology Division, School of Clinical Sciences, Monash Health, Monash University, Melbourne, Australia

5 Precision Medicine, School of Clinical Sciences at Monash Health, Monash University, Melbourne, Australia

6 Department of Lung and Sleep, Monash Medical Centre, Clayton, Australia

7 School of Clinical Sciences, Monash University, Melbourne, Australia

8 Sleep and Circadian Rhythm Laboratory, Monash University, Melbourne, Australia

9 Department of Respiratory and Sleep Medicine, Eastern Health, Melbourne, Australia

10 Eastern Health Clinical School, Monash University, Melbourne, Australia

11 School of Agriculture and Food, Melbourne University, Melbourne, Australia

Personalised weight loss treatment requires reliable biomarkers of treatment response. This study assessed whether transcriptomic analysis could predict treatment response. The discovery dataset contained men with obstructive sleep apnoea $(n=18$, OSA) undergoing a weight loss program. High responders (HR) were defined as $\geq 5 \%$ decrease in weight and waist circumference and a decrease in OSA severity. Mixed responders (MID) met one or two criteria, and low responders (LR) met none. Linear mixed modelling determined differentially expressed transcripts (adjusted $p<0.05$ ). Four transcripts were significantly downregulated in HR compared to MID and LR: ELANE (logFC: -2.8 vs. LR and -2.2 vs. MID), OLFM4 (logFC: -6.4 vs. LR and -5.4 vs. MID), DEFA3 (logFC: -4.0 vs. LR and -4.53 vs. MID), and MS4A3 (logFC: -3.2 vs. LR and -2.9 vs. MID). Logistic regression models predicted the probability of HR based on initial weight and ELANE, OLFM4, DEFA3, MS4A3 in an independent dataset with overweight or obesity $(n=38)$. The best fit model contained initial weight and OLFM4. Treatment response was predicted with $61.1 \%$ accuracy, $41.67 \%$ sensitivity, and $100 \%$ specificity. These four genes are promising predictors of weight loss response. The results should be confirmed in a larger, more diverse population.

3.57. Associations between Bitter and Sweet Perception and Self-Reported Oral Hygiene Habits: A Cross-Sectional Survey

Kiranjit Kaur ${ }^{1}$, Dean Sculley ${ }^{2}$, Martin Veysey ${ }^{3,4}$, Mark Lucock ${ }^{1}$, Janet Wallace ${ }^{5,6}$ and Emma Beckett $1,7,8$

1 School of Environmental and Life Sciences, University of Newcastle, Newcastle, Australia

2 School of Biomedical Sciences and Pharmacy, University of Newcastle, Newcastle, Australia

3 School of Medicine and Public Health, University of Newcastle, Newcastle, Australia

4 Hull-York Medical School, University of York, Heslington, UK

5 School of Health Sciences, University of Newcastle, Newcastle, Australia

6 School of Dentistry, Faculty of Medicine and Health, University of Sydney, Sydney, Australia

7 Hunter Medical Research Institute, University of Newcastle, Newcastle, Australia

8 Priority Research Centre for Physical Activity and Nutrition, University of Newcastle, Callaghan, Australia

Oral diseases and diet link bidirectionally. Taste perception and genetics may also be involved in modulating this relationship through altering diet and oral inflammation. 
Sweet and bitter taste genetics have been linked to risk for dental caries and inflammation. However, the relationship between taste perception and oral hygiene habits has not been investigated. An online cross-sectional survey was conducted to investigate links between bitter and sweet taste (liking/intensity using General Labelled Magnitude Scale and index foods) and oral hygiene habits $(n=518)$, with adjustments for age, sex, income, education and dietary habits. Least-squares means were compared (ANOVA: Tukey HSD's post-hoc). Higher bitter intensity $(16-21 \%)$ was associated $(p<0.0001)$ with more regular brushing, mouthwash use, chewing gum and tongue cleaning. Higher sweetness intensity was related to more regular mouthwash use, brushing and chewing gum $(p<0.001)$. Higher sweet liking was related to more regular mouthwash use $(p=0.03)$. Lower bitter liking was associated with more regular brushing $(p=0.004)$, chewing gum $(p=0.008)$, mouthwash $(p=0.007)$ and floss use $(p=0.01)$. Findings suggest relationships between bitter and sweet perceptions, oral hygiene habits and oral health, which justify the need for future studies to assess consequences.

\title{
3.58. Relationships between Bitter Taste, Health Values, and Vegetable Perception and Consumption
}

\section{Melanie Cockbain ${ }^{1,2}$, Tamara Bucher ${ }^{1,2}$ and Emma L. Beckett ${ }^{1,2}$ \\ 1 University of Newcastle, Ourimbah, Australia \\ 2 Priority Research Centre for Physical Activity and Nutrition, The University of Newcastle, Callaghan, Australia}

Under $10 \%$ of Australians meet the recommended 5 daily serves of vegetables. Understanding factors that influence vegetable consumption may help to inform future strategies for increasing consumption. While some factors have been identified, including taste and personal values, the relationship between these factors, or the additive and interactive effects they may have on vegetable consumption, remains unclear. Therefore, a crosssectional survey relating to vegetable consumption (variety and frequency), perception (healthfulness and tastiness; 100-point scale), health values (5-point Likert scales), and bitter taste (index foods; liking and intensity; 100-point scale) was conducted. The results from 512 Australian adults showed that participants with higher bitter liking score were more likely to have higher vegetable perception scores $(p<0.001)$. Similar results were recorded for participants with higher health values $(p<0.01)$. Participants with higher perception scores also had higher consumption $(p<0.0001)$ and variety scores $(p<0.01)$. Participants with higher health value scores were more likely to report a higher bitter liking $(p<0.05)$. These results suggest complex associations between bitter taste, health values, vegetable perception and consumption. Further research is needed to identify the nature of this relationship, and how factors may interact to predict vegetable consumption.

\subsection{Superfood Extracts and Their Potential Health Benefits}

\author{
Jacqueline Barsby ${ }^{1,2}$, James Cowley ${ }^{1}$, Shalem Leemaqz ${ }^{2,3}$, Jessica Grieger 2,4, \\ Daniel McKeating ${ }^{5}$, Tony Perkins ${ }^{5}$, Susan Bastian ${ }^{1}$, Rachel Burton ${ }^{1}$ and \\ Tina Bianco-Miotto ${ }^{1,2}$ \\ 1 School of Agriculture, Food and Wine, The University of Adelaide, Urrbrae, Australia \\ 2 Robinson Research Institute, Adelaide, Australia \\ 3 College of Medicine and Public Health, Flinders University, Bedford Park, Australia \\ 4 Adelaide Medical School, The University of Adelaide, Adelaide, Australia \\ 5 School of Medical Sciences, Griffith University, Gold Coast Campus, \\ Parklands Drive, Australia
}

There is a pressing need for nutritious food sources containing quality proteins, fats, and carbohydrates, resulting in a rise in foods claiming to meet these criteria. Many of these foods are termed 'superfoods' in the media, with claims that they can reduce the risk of chronic diseases such as arthritis, asthma, and bowel disease and improve overall human health. These claims are often unfounded, and the benefits of 'superfoods' are 
largely unknown. The objective of this study was to investigate the nutritional properties of superfood seeds: flax, chia, hulled sunflower, and two types of processed hemp seeds and determine whether they may have potential health benefits. Crude aqueous extracts were prepared from seeds, and their composition assessed was by mineral, protein, and monosaccharide analyses. IEC6 and CACO-2 cells were used to test the impact of the extracts on cell viability. Increased cell viability was observed in both cell lines with increasing concentrations of the flax, chia, or sunflower extracts $(p<0.05)$. Compositional analyses revealed the presence of polysaccharides, proteins, and essential minerals in the seed extracts, and in vitro assays showed that sunflower had increased antioxidant activity. Future studies are needed to further characterise these and other superfoods and investigate their potential health benefits

\subsection{Gluten-Free Seed Mix Favours Butyrate Production during In Vitro Human Faecal Fermentation}

\section{Damien P. Belobrajdic, Jennifer Giles and Bruce May \\ CSIRO, Adelaide, Australia}

Dietary eating patterns that are low in dietary fibre, including avoidance of gluten or low FODMAP, can adversely affect gut microbial composition and lower the microbial production of short chain fatty acids (SCFA). To address this, a gluten free, high fibre, low FODMAP seed mix (Blend11) was developed, and its fermentability was assessed using in vitro methodology. Blend11 (raw and soaked in water for up to $72 \mathrm{~h}$ ), cellulose and psyllium husks underwent pre-digestion prior to in vitro fermentation in media containing human faeces, with SCFA quantified at $0,5,10$ and $24 \mathrm{~h}$. The fermentation of Blend11 produced more SCFA than psyllium at $10 \mathrm{~h}(p<0.05)$, but after $24 \mathrm{~h}$, the concentrations of total SCFA were similar. Throughout the 24-h fermentation period, Blend11 produced more butyrate than psyllium or cellulose (both $p<0.05$ ), and differences in fibre composition such as higher resistant starch levels in Blend11 may have contributed to these higher levels. Soaking did not affect the fermentability of Blend11. These results suggest that Blend11 contains fibres that are fermented by the human faecal microbiota through increasing SCFA concentration, and that butyrate production is favoured, but confirmatory in vivo dietary intervention studies are needed.

3.61. The FoodChecker Study: Exploring Use of a Digital Tool to Support Healthy Food Provision in Victorian Long Day Care Services

\section{Jessica V. Kempler ${ }^{1}$, Penny Love ${ }^{1}$, Kristy Bolton ${ }^{1}$, Margaret Rozman ${ }^{2}$ and Alison Spence ${ }^{1}$ \\ 1 School of Exercise and Nutrition Sciences, Deakin University, Geelong, Australia \\ 2 Healthy Eating Advisory Service, Nutrition Australia, Melbourne, Australia}

Digital tools provide a unique opportunity to support the implementation of food provision guidelines for Long Day Care (LDC); however, little is known about their adoption and use. This study explored LDC service experiences with FoodChecker, the Victorian government funded digital menu planning and assessment tool to support food provision according to Victorian Menu planning guidelines for long day care (MPGs). A qualitative cross-sectional survey of LDC directors and cooks asked about user experiences and attitudes, with prompts about outcomes of FoodChecker use and barriers and enablers to use. Inductive thematic analysis was conducted. Preliminary data from 35 respondents identified common themes of FoodChecker usefulness in supporting children's health, improving menu planner confidence and knowledge and enabling centre practices to align with the MPGs. Time and management encouragement were considered important factors influencing FoodChecker use. Participants identified that strategies to reduce data input time and provision of additional resources such as recipes and sample menus would improve FoodChecker's usefulness. As the first qualitative study exploring LDC staff experiences with a digital menu planning tool, these findings will inform further research 
and refinement of FoodChecker, thus aiding scalable and sustainable support to improve healthy food provision for children in LDC services.

\subsection{Enabling Better Nutrition for Adolescents from Middle Eastern Backgrounds}

Nema Hayba, Yumeng Shi and Margaret Allman-Farinelli

University of Sydney, Camperdown, Australia

Parents remain important stakeholders in shaping adolescent eating behaviours. This qualitative study gathered the opinions of Middle Eastern (ME) parents residing in Australia on barriers and enablers to healthy eating interventions for ME adolescents given the high prevalence of obesity among the community. Semi-structured interviews were conducted with $26 \mathrm{ME}$ parents (female) aged 35-59 years and most residing in socioeconomically disadvantaged areas $(n=18)$. A reflexive thematic analysis using the Capability, Opportunity, Motivation-Behaviour model and Theoretical Domains Framework for coding was performed. Parents voiced confidence in adolescent's knowledge of the importance of healthy eating but were less optimistic this was practiced in environments outside of parental influence. Time management was a barrier for parents to support healthy eating with some reliance on the nearby plentiful fast-food outlets. This increased via home delivery during COVID-19. Breakfast skipping was not uncommon due to time constraints. A culture of feeding compensating for intergenerational trauma experienced by this diaspora was also identified. Parents expressed concern for adolescent's eating behaviours and believed extra support in the form of government and school policies such as further regulation of fast-food and subsidization of heathy food products were needed. Opportunities for targeted programs that included parenting workshops were recommended.

\subsection{Do Regulated or Unregulated Claims Influence Parent Perceptions of Healthiness? A Discrete Choice Experiment with Toddler Foods}

\section{Jennifer McCann ${ }^{1}$, Julie Woods ${ }^{1}$, Mohammadreza Mohebbi ${ }^{2}$ and Georgie Russell ${ }^{1}$}

1 School of Exercise and Nutrition Sciences, Deakin University, Geelong, Australia

2 Biostatistics Unit, Faculty of Health, Deakin University, Burwood, Australia

Background: Sales and consumption of toddler foods, the majority of which are discretionary and ultra-processed, have increased over time. These foods display numerous on-package nutrition and promotional claims, which have been shown to impact consumer's food perceptions and choices. Aim: To investigate the relative impact of different regulated and unregulated claims on parent perceptions of the healthiness of an ultraprocessed, discretionary toddler snack food in Australia. Methods: Adults aged 18 and over completed an online discrete choice experiment. Participants chose the most and least healthy product based over seven choice sets. Each product had a combination of three different types of claims. Data were analysed using an ordinal logistic regression model. Results: Participants were more than 13 times more likely to select a product with the regulated nutrient content claim "no added sugar, no added salt" as most healthy (OR 13.71; CI: 10.93-17.22, $p<0.001$ ), compared to no regulated nutrient content claim (reference category). Conclusions: Regulated claims were the most influential claim type on parental perceptions of healthiness of a toddler snack food, this should be addressed to increase the salience of regulated claims in Australia. Further regulatory controls are required to provide accurate nutrition information and facilitate informed consumer choice.

\subsection{The Effect of Downsizing Discretionary Food Packages on Consumers' Food-Related Behaviours, Intentions, and Perceptions-A Scoping Review}

\section{Qingzhou Liu, Lok Yin Tam and Anna Rangan}

Charles Perkins Centre, School of Life and Environmental Sciences, Faculty of Science, University of Sydney, Sydney, Australia

Single-serve packaging of discretionary foods is becoming increasingly popular, but evidence is limited on whether smaller package sizes can reduce food intake. The aim 
of this scoping review was to assess the effect of reducing package size of discretionary foods on consumers' food-related behaviours, intentions, and perceptions. The search was conducted in six databases and grey literature sources following the PRISMA Scoping Review guidelines. After screening 5562 articles, 30 articles comprising 47 intervention studies were eligible. Most studies were set in a laboratory (79\%) and had a homogenous study population ( $53 \%$ university students). Twelve of 15 studies found a significant effect in lowering actual or intended consumption when a single smaller package was offered compared with a single larger package. When the total serving size was held constant between varying package conditions, such as multi-pack, single-serve pack or unpackaged, the results on actual and intended consumption were inconsistent and varied according to presence of moderators. Overall, these findings suggest that reducing the size of a single package is the most promising approach for improving portion control. The benefit of offering discretionary foods in smaller multipacks versus those in larger packages or unpackaged is less convincing and requires further study.

3.65. What Can Social Media Tell Us about Food Security? A Sentiment Analysis of Food Security Social Media Data

\section{Annika Molenaar ${ }^{1}$, Dickson Lukose ${ }^{2}$, Linda Brennan ${ }^{3}$, Eva L. Jenkins ${ }^{1}$ and Tracy A. McCaffrey ${ }^{1}$ \\ 1 Department of Nutrition, Dietetics \& Food, Monash University, Notting Hill, Australia \\ 2 Monash Data Futures Institute, Monash University, Clayton, Australia \\ 3 School of Media \& Communication, RMIT University, Melbourne, Australia}

Food-security-related information on social media remains largely unexplored. Sentiment analysis can be performed on large datasets such as social media and provide an understanding of the public discourse around issues such as food security. This study aimed to explore the sentiment of the conversation around food security on social media. Search terms around food security were iteratively refined to collect Tweets relevant to food security. Data from 2019 to 2020 were collected through the Twitter API. Valence Aware Dictionary and Sentiment Reasoner (VADER) sentiment analysis was used to analyse the Tweets. A total of 13,215,401 Tweets were collected globally and 124,544 Tweets were from Australia only. Of the Australian Tweets, 39\% were from the year 2019 while $61 \%$ were from 2020. From the years 2019 to 2020, $44.3 \%$ of Tweets were classified as positive, $29.7 \%$ as neutral, $22.9 \%$ as negative, $2.0 \%$ as very positive and $1.1 \%$ as very negative. In conclusion, more Australian Tweets around food security were sent in 2020 than 2019, and the majority had a positive or neutral sentiment and were less commonly negative. To obtain a more nuanced understanding of the sentiment and content of the social media data, additional computer science analysis should be performed.

3.66. Salt-Related Knowledge, Attitudes and Behaviours (KABs) among Parents and Caregivers following a State-Wide Salt Reduction Intervention in Victoria, Australia

Carley Grimes ${ }^{1}$, Kristy Bolton ${ }^{1}$, Durreajam Khokhar ${ }^{1}$, Kathy Trieu ${ }^{2}$, Jenny Reimers ${ }^{3}$, Sian Armstrong ${ }^{4}$, Bruce Bolam ${ }^{5}$, Joseph Alvin Santos ${ }^{6}$,

Emalie Rosewarne ${ }^{6}$, Elizabeth Dunford ${ }^{6,7}$, Stephan Jan ${ }^{6}$, Mark Woodward ${ }^{6,8}$, Bruce Neal ${ }^{6,8}$, Caryl Nowson ${ }^{1}$ and Jacqui Webster ${ }^{6}$

1 Institute for Physical Activity and Nutrition, School of Exercise and Nutrition Sciences, Geelong, Australia

2 The George Institute for Global Health, University of New South Wales, Sydney, Australia

3 Victorian Health Promotion Foundation (VicHealth), Melbourne, Australia

4 Heart Foundation, Melbourne, Australia

5 Department of Health and Human Services, Melbourne, Australia

6 The George Institute for Global Health, University of New South Wales, Newtown, Australia 
7 Department of Nutrition, Gillings Global School of Public Health, The University of North Carolina, Chapel Hill, USA

8 The George Institute for Global Health, Imperial College of London, London, UK

As part of a state-wide salt reduction intervention, two public awareness campaigns were delivered by the Heart Foundation during 2016-19: 'Don't trust your taste buds' and 'Unpack the Salt'. This study assessed if salt-related KABs among parents/caregivers changed post-intervention. Cross-sectional surveys of adults aged 18-65 years who cared for children $<18$ years were conducted before (2015) and after (2019) the intervention. Participants were recruited from consumer research panels, Facebook and four shopping centres. The online survey was completed by 1746 parents/caregivers ( $55 \%$ female, $41 \mathrm{y})$ (baseline $n=821$; follow-up $n=925$ ). Post-intervention, there was no change in the percentage of parents/caregivers that knew children eat too much salt $(72 \% \mathrm{vs} .69 \%$, $p=0.30)$ and that this can be harmful to children's health (77\% vs. $76 \%, p=0.08)$. Conversely, significantly fewer parents reported placing a salt shaker on the table during meal times $(-10 \%, p<0.001)$ and that their child added salt at the table $(-7 \%, p=0.04)$. There was no change in parents / caregivers reported use of salt added to meals prepared for children. These findings suggest that the public awareness campaign did not impact on the already high knowledge parents/caregivers have related to children's salt consumption, however, there were some small improvements in discretionary salt use behaviours.

\title{
3.67. Sensors for Identifying Food Consumption and Time of Intake; a Scoping Review
}

\author{
Leanne Wang ${ }^{1}$, Margaret Allman-Farinelli ${ }^{1}$, Jiue-An Yang ${ }^{2}$, Luke Gemming ${ }^{1}$, \\ Jennifer Taylor ${ }^{3}$, Eric Hekler ${ }^{3,4}$ and Anna Rangan ${ }^{1}$ \\ 1 Charles Perkins Centre, Faculty of Medicine and Health, University of Sydney, \\ Sydney, Australia \\ 2 Department of Population Sciences, Beckman Research Institute, City of Hope, \\ Duarte, USA \\ 3 The Design Lab, University of California, San Diego, USA \\ 4 Herbert Wertheim School of Public Health and Human Longevity Science, University \\ of California, San Diego, USA
}

As dietary patterns shift towards less structured patterns of eating, passive technologybased dietary assessment methods are required to capture frequently omitted snacks and smaller meals and meal times. The aim of this study was to identify objective measures of dietary intake using sensor-based technologies. A scoping review was conducted using the PRISMA extension for scoping reviews framework. All studies evaluating the performance of sensor-based devices in identifying and recording food intake times published between 2016 and 2021 were extracted from seven databases and charted. A total of 68studies describing 54 unique devices worn on the wrist $(n=16)$, head $(n=16)$, neck $(n=9)$, and other locations $(n=13)$ were included. With feasibility for real-world use being defined as having high accuracy in detecting meals, snacks, and beverages; a long battery life; and being socially acceptable and comfortable to wear, few devices identified in the scoping review met this definition. The main limitations were devices missing snacks, poor performance translating from laboratory to free-living settings, and being visually obtrusive, compromising user comfort and acceptance. Further refinement to both the hardware and performance of these devices is required before sensor-based dietary assessment tools can be used by dietitians in practice settings.

3.68. Nutrition Policy Environments in Victorian Long Day Care Centres-Do They Align with Best Practice?

\author{
Ganna Aristova ${ }^{1}$, Alison Spence ${ }^{2}$, Audrey Elford ${ }^{2}$, Laura Graham ${ }^{1}$ and \\ Penelope Love ${ }^{2}$ \\ 1 School of Exercise and Nutrition Science (SENS), Deakin University, Geelong, Australia
}


2 Institute of Physical Activity and Nutrition (IPAN), SENS, Deakin University,

Geelong, Australia

Childcare centres provide an opportune setting for widespread health promotion to develop the healthy eating behaviours of children. Policies are essential as they provide streamlined nutrition guidance for centre staff; however, the literature assessing nutrition policy environments in Australian long day care centres (LDCs) is scarce. This study examined the centre-based nutrition policies of LDCs in Victoria, Australia, using the WellCCAT tool. A full policy analysis was conducted for each centre by two independent researchers to assess the comprehensiveness (scope) and strength of nutrition policy guidance across the four domains-Nutrition Education (NE), Nutrition Standards (NS), Health Promotion (HP) and Communication and Evaluation (CE). Preliminary data from 19 LDCs showed low average total comprehensiveness scores $(50 / 100)$ with highest component scores for NE $(67 / 100)$ and HP $(64 / 100)$. Average total strength scores were very low $(15 / 100)$ with highest component score for CE $(12 / 100)$. Centres in the highest SEIFA decile $(n=8)$ and privately managed centres $(n=12)$ scored highest for overall comprehensiveness and strength. The results indicate a need for centre-based policies to broaden their scope and strengthen their content and language. Additional research is needed to enhance the development and assessment of centre-based policy to enable supportive nutrition environments in LDCs.

\title{
3.69. Menu Planning in Childcare: The Role of Government Support Services
}

Audrey Elford ${ }^{1}$, Alison Spence ${ }^{1}$, Amy Wakem ${ }^{2}$, Karen Campbell ${ }^{1}$ and Penny Love ${ }^{1}$

1 Institute of Physical Activity and Nutrition, School of Exercise and Nutrition Science,

Geelong, Australia

2 Healthy Eating Advisory Service, Nutrition Australia, Melbourne, Australia

Long day care centres (LDCs) are important settings for healthy food provision to children, but research consistently indicates that LDC menus are not compliant with dietary guidelines. Whilst some government-funded services are in place to support menu compliance, the value of these to address barriers is unexplored. This study aimed to assess relationships between LDCs characteristics, support access, perceived barriers/enablers to menu planning, and menu compliance. Victorian LDCs $(n=89)$ completed a survey assessing barriers/enablers based on the Theoretical Domains Framework (TDF). A subset $(n=18)$ provided 2-week menus, which were assessed for menu compliance. Just over half of all centres accessed the Victorian-government-funded service for menu planning, the Healthy Eating Advisory Service (HEAS). Accessing HEAS support was associated with higher average scores for the TDF domains knowledge $(p<0.01)$, optimism $(p=0.04)$, skills $(p=0.03)$, and reinforcement $(p=0.05)$. Just one centre met menu compliance. While government-funded support services may help LDCs address some barriers to menu planning, this may not translate into menu compliance. Future research is needed to understand the complexities of food provision and menu compliance to inform practical strategies and public health nutrition interventions for the LDC sector.

3.70. Investigating the Efficacy and Feasibility of Using a Whole-of-Diet Approach to Lower Circulating Levels of C-Reactive Protein in Postmenopausal Women with Abdominal Obesity. A Mixed Methods Pilot Study

\author{
Stephanie Cowan ${ }^{1}$, Aimee Dordevic ${ }^{1}$, Andrew Sinclair ${ }^{1}$, Helen Truby ${ }^{2}$, \\ Surbhi Sood ${ }^{1}$ and Simone Gibson ${ }^{1}$ \\ 1 Monash University, Clayton, Australia \\ 2 The University of Queensland, Brisbane, Australia
}

Chronic inflammation is an underlying pathophysiology for cardiovascular disease development in postmenopausal women. This mixed-methods pilot study aimed to determine the feasibility and efficacy of an anti-inflammatory whole-of-diet intervention 
in weight-stable postmenopausal women with abdominal obesity $(n=13)$. Quantitative outcomes included change in inflammatory and metabolic markers from baseline to four weeks. Focus groups undertaken at the end of the study were thematically analysed to explore participants' lived experience. Plasma insulin and HOMA-IR significantly decreased by $0.90(-0.05-2.20) \mathrm{mmol} / \mathrm{L}$ and $0.29(-0.03-0.59)$, respectively ( $p \leq 0.023$ for both). In participants with subclinical inflammation at baseline (CRP $\geq 3 \mathrm{mg} / \mathrm{L}$ ), levels decreased by $1.7(1.2-3.6) \mathrm{mg} / \mathrm{L}(p=0.068)$ without significant change to anthropometric measures. The focus groups revealed that post-menopause is a transitional period characterised by increased capacity for behavioural change. Participants were highly engaged with learning about innovative nutrition topics, favouring a comprehensive education style. Dietary interventions that target inflammation, irrespective of weight loss, may be a viable strategy for CVD risk reduction in postmenopausal women with subclinical inflammation. Though education must be delivered at a level of complexity that can satisfy the learning needs of postmenopausal women, who display proficient health literacy and cooking skills.

\title{
3.71. Factors Influencing Food Consumption at Eating Occasions: A Systematic Review
}

\author{
Nancy Tran ${ }^{1}$, Rebecca Leech ${ }^{1}$, Emily Denniss ${ }^{1}$, Kentaro Murakami ${ }^{2}$ and \\ Sarah A. McNaughton ${ }^{1}$ \\ 1 Institute for Physical Activity and Nutrition (IPAN), School of Exercise and Nutrition \\ Sciences, Faculty of Health, Deakin University, Geelong, Australia \\ 2 Department of Social and Preventive Epidemiology School of Public Health, \\ University of Tokyo, Tokyo, Japan
}

Understanding the determinants of eating patterns or food consumption at eating occasions is critical for the development of interventions to address diet-related chronic diseases. A systematic review search of three databases (MEDLINE Complete, PsycINFO and EMBASE) was conducted to identify studies examining potential correlates of food consumption at different eating occasions in free-living populations greater than two years of age (PROSPERO no.: CRD42021219798). A total of 4836 articles were identified, with 228 full-text articles assessed for eligibility, resulting in 66 included studies. Most studies used a cross-sectional design $(n=59)$, with only seven having longitudinal designs. In terms of the studied population, 34 consisted of children and adolescent participants, 25 included adults only and 7 included both children and adults. Most studies investigated both meals and snacks $(n=26)$, followed by breakfast, snack, lunch or a neutral eating occasion term where the eating occasion was not classified as a meal or a snack $(13,12,9$ and 6 , respectively). In total, 41 studies assessed interpersonal correlates (i.e., age, gender), 11 assessed social contextual correlates (i.e., person presented) and 14 assessed environmental contextual correlates (i.e., location). This review provides a summary of the available evidence surrounding food consumption at eating occasions across children and adults.

\subsection{Young Australian Adults' Perceptions of the Term 'Discretionary Food'}

\section{Krupa T. Kombanda, Claire Margerison, Alison Booth and Anthony Worsley}

Institute for Physical Activity and Nutrition, School of Exercise and Nutrition Sciences, Deakin University, Geelong, Australia

The Australian Dietary Guidelines refer to energy dense and nutrient poor foods as 'discretionary food'. However, a growing body of evidence suggests the term is not accurately understood among Australians. In particular, we know little about how young Australian adults resonate with the term 'discretionary food'. To contribute understanding to this issue, we explored 19-30-year-old young Australian adults' knowledge and awareness of the term 'discretionary food' focussing on any differences across groups in different living arrangements. Through a social-constructivist approach, young adults $(n=38)$ were interviewed Australia-wide using online methods or over the phone. Data were thematically analysed. Less than one-half of the participants $(n=8)$ understood the term 'discretionary food'. 'Junk food' was the most popular term used by one-half of the 
participants $(n=17)$ to refer to unhealthy foods. Misalignment and misunderstanding was consistent across groups of young adults residing in different living arrangements. Our findings suggest the need for improved clarity and consistency surrounding the term 'discretionary food'. The use of simpler and more familiar terms that closely resonate with this age cohort may help to increase uptake and utility in efforts to reduce young adults' high consumption of 'discretionary food'.

3.73. Associations between Empirical Dietary Patterns and Sociodemographic Characteristics and Obesity in Iranian Adults

\section{Sara Ebrahimi ${ }^{1}$, Sarah A. McNaughton ${ }^{1}$, Rebecca M. Leech ${ }^{1}$, Morteza Abdollahi ${ }^{2}$, Anahita Houshiarrad ${ }^{2}$ and Katherine M. Livingstone ${ }^{1}$ \\ 1 Institute for Physical Activity and Nutrition, School of Exercise and Nutrition Sciences, Deakin University, Geelong, Australia \\ 2 Department of Nutrition Research, National Nutrition and Food Technology Research Institute, School of Nutrition Sciences and Food Technology, Shahid Beheshti University of Medical Sciences, Tehran, Iran}

Research examining the associations between dietary patterns (DPs) and obesity in Iranian adults is limited by small and non-representative samples. This study examined associations between DPs and sociodemographic characteristics and body mass index (BMI) in a nationally representative sample of Iranian adults. Household dietary intake, collected using three 24-h recalls from 6833 households, was analysed using principal component analysis to derive DPs. Linear and multi-level linear regression models were used to evaluate associations between household DPs and adults' sociodemographic characteristics and BMI, respectively. Three DPs were identified. The first and third DPs were characterized by higher intake of fruits, vegetables, non-hydrogenated fats, cakes, and soft drinks, while the second DP was characterized by higher intake of bread, legumes, hydrogenated fats, and onions. The second DP was similar to a traditional Iranian diet and was associated with lower education and living in rural areas. All three DPs were associated with BMI: first pattern ( $\beta$ : 0.49 ; 95\% confidence interval: $0.43,0.55)$, second pattern $(0.17 ; 0.09,0.25)$, and third pattern $(0.17 ; 0.11,0.24)$. The results suggest that while all three DPs were associated with higher BMI, households consuming these diets varied. Longitudinal individual dietary intake data are needed to further explore these results.

\subsection{An Audit of Available Australian Convenience Cooking Products and Their Vegetable Content and Vegetable Varieties}

\section{Natasha Brasington ${ }^{1}$, Patrice Jones ${ }^{1,2}$, Melissa Garland ${ }^{1}$, Tamara Bucher ${ }^{1,3}$ and Emma L. Beckett 1,2,3 \\ 1 Environmental and Life Sciences, The University of Newcastle (Central Coast), Ourimbah, Australia \\ 2 Hunter Medical Research Institute, New Lambton Heights, Australia \\ 3 Priority Research Centre for Physical Activity and Nutrition, The University of Newcastle, Callaghan, Australia}

Most of the Australian population fail to meet the recommended levels of vegetable intake, with new approaches needed to improve the population's vegetable intake. Convenience cooking products (meal and recipe base products) are regularly utilised by Australians. Although these products are thought to be non-nutritious, they are low-cost, require little preparation time and cooking skills, and included back-of-pack recipes could provide a new pathway for encouraging vegetable consumption if balanced. However, the vegetable content of back-of-pack recipes included in this category of products has not been collectively audited. An audit of the recipes provided on meal/recipe base products $(n=91)$ sold in major Australian supermarkets was therefore conducted. There was an average of 1.58 standard serves of vegetables per back-of-pack recipe serve, with $75 \%$ of recipes providing $<2$ standard serves of vegetables. Recipes had low vegetable variety, 
comprised primarily of red-orange, starchy and "other" vegetables (average $0.43,0.41$, and 0.56 standard serves, respectively), with limited green vegetables and legumes (average 0.9 and 0.10 standard serves). The results provide insights into the vegetable content of the recipes on meal/recipe base products sold in Australia and highlight how these recipes could be adjusted to increase vegetable intakes in users.

Funding: The publication of these proceedings received no external funding.

Conflicts of Interest: The authors declare no conflict of interest. 